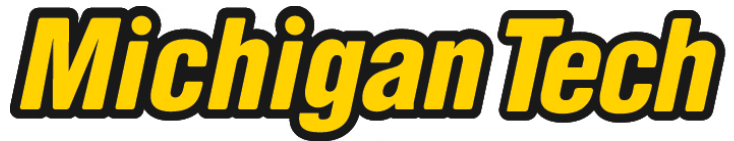 \\ Michigan Technological University Create the Future Digital Commons @ Michigan Tech
}

velocity CFD approaches

Anurag Rajan

Michigan Technological University

Follow this and additional works at: https://digitalcommons.mtu.edu/etds

Part of the Mechanical Engineering Commons

Copyright 2011 Anurag Rajan

\section{Recommended Citation}

Rajan, Anurag, "Effect of mesh distortion on the accuracy of high order vorticity-velocity CFD approaches", Master's Thesis, Michigan Technological University, 2011.

https://doi.org/10.37099/mtu.dc.etds/402

Follow this and additional works at: https://digitalcommons.mtu.edu/etds

Part of the Mechanical Engineering Commons 


\title{
EFFECT OF MESH DISTORTION ON THE ACCURACY OF HIGH ORDER VORTICITY-VELOCITY CFD APPROACHES
}

\author{
By \\ ANURAG RAJAN \\ A THESIS \\ Submitted in partial fulfillment of the requirements \\ for the degree of \\ MASTER OF SCIENCE \\ MECHANICAL ENGINEERING
}

MICHIGAN TECHNOLOGICAL UNIVERSITY

2011

(C) 2011 Anurag Rajan 
This thesis, "Effect of Mesh Distortion on the Accuracy of High Order Vorticity-Velocity CFD Approaches," is hereby approved in partial fulfillment of the requirements for the degree of MASTER OF SCIENCE in Mechanical Engineering.

Department of Mechanical Engineering-Engineering Mechanics

Signatures:

Thesis Advisor

Dr. Fernando Ponta

Department Chair

Dr. William Predeborn

Date 


\section{Contents}

List of Figures $\ldots \ldots \ldots \ldots \ldots \ldots \ldots \ldots \ldots \ldots$ vi

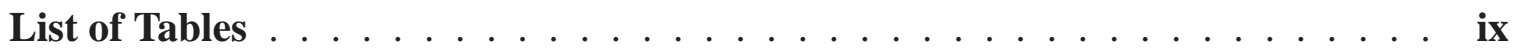

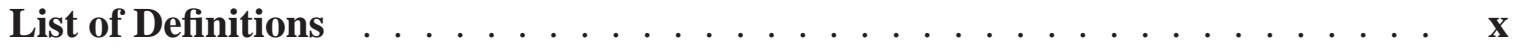

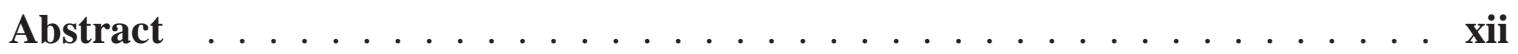

1 Introduction $\ldots \ldots \ldots \ldots \ldots \ldots \ldots \ldots \ldots \ldots$

1.1 Fluid structure interaction $\ldots \ldots \ldots \ldots \ldots \ldots \ldots$

1.2 An emerging field of application:The wind power challenge . . . . . . . 6

1.3 Thesis outline $\ldots \ldots \ldots \ldots \ldots \ldots \ldots \ldots \ldots \ldots$

2 Numerical methods $\ldots \ldots \ldots \ldots$. . . . . . . . . . . . . . . . . . 11

2.1 The Navier - Stokes equations . . . . . . . . . . . . . . . . . . . . . 11

2.2 Numerical Solution of the incompressible viscous flow $\ldots \ldots \ldots$

2.2.1 Primitive variables . . . . . . . . . . . . . . . . . . . . . . . 14

2.2.1.1 Non-fractional step methods . . . . . . . . . . . . 17

2.2.1.2 Fractional step methods . . . . . . . . . . . . . . 19

2.2.1.3 Artificial incompressibility . . . . . . . . . . . . . . 23

2.2.2 Non-Primitive variables . . . . . . . . . . . . . . . . . . . . 24 
2.2.2.1 Vorticity - stream function formulation for two dimensional

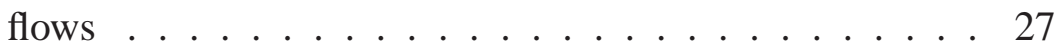

2.2.2.2 Biharmonic formulation . . . . . . . . . . . . . 29

2.2.2.3 Coupled formulation in vorticity and Stream function . . . 30

2.2.2.4 Uncoupled formulation using vorticity integral conditions 30

3 Hybrid methods $\ldots \ldots \ldots \ldots \ldots \ldots$

3.1 Introduction . . . . . . . . . . . . . . . . . . . . 34

3.1.1 Equations in three dimensions $\ldots \ldots \ldots \ldots$

3.1.2 Equations in two dimensions . . . . . . . . . . . . . . . 39

3.2 The Kinematic Laplacian equation method . . . . . . . . . . . . . . . . 41

3.2.1 The constant-curl Laplacian equation $\ldots \ldots$. . . . . . . . . . 41

3.2.2 A generalized Laplacian $(\omega, \mathbf{u})$ method: The KLE $\ldots \ldots \ldots$. . . . 42

3.2.3 Variational formulation of KLE . . . . . . . . . . . . . . 47

4 Numerical implementation of the KLE $\ldots \ldots \ldots \ldots$

4.1 Introduction $\ldots \ldots \ldots \ldots \ldots \ldots \ldots \ldots \ldots$

4.2 The Spectral-element method for KLE . . . . . . . . . . . . . . . 52

5 Experiment and analysis $\ldots \ldots \ldots \ldots \ldots \ldots$

5.1 Introduction $\ldots \ldots \ldots \ldots \ldots \ldots \ldots \ldots \ldots \ldots \ldots \ldots \ldots \ldots$

5.2 Controlled distortion experiments . . . . . . . . . . . . . . . . . 64

5.2.1 The canonical test problem . . . . . . . . . . . . . . 65 
5.2 .2 The experiment . . . . . . . . . . . . . 67

5.3 Observations and analysis . . . . . . . . . . . . . . 70

5.3.1 Error analysis ..................... 70

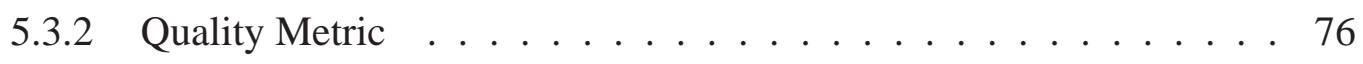

5.4 Improved tri-quadrilateralization $\ldots \ldots$. . . . . . . . . . 86

Appendix A $\ldots \ldots \ldots \ldots \ldots$

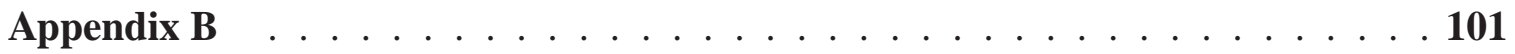

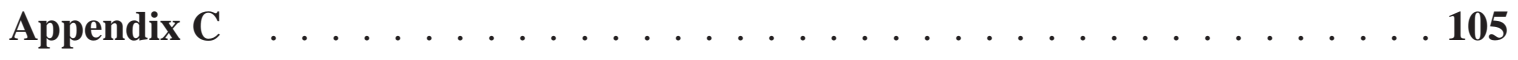

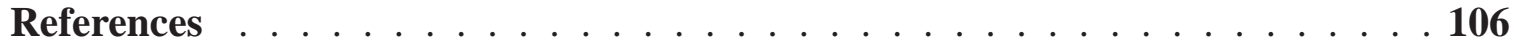




\section{List of Figures}

1.1 The REpower M5 5-megawatt turbine, with a rotor diameter of approximately 126 meters. . . . . . . . . . . . . . . . . . . . 7

4.1 A two-dimensional nine node isoparametric element in its natural coordinate system along with a graphical representation of three of its nine inter-

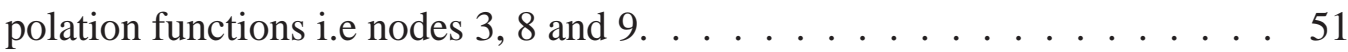

4.2 A tri-quadrilateral finite element mesh derived from an unstructured triangular mesh. . . . . . . . . . . . . . . . 57

4.3 The internal topology of a tri-quadrilateral element. Quadrilateral elements (I)-(III) are the nine-node isoparametric elements. 1-19 is the in-triangle global numbering of the nodes. . . . . . . . . . . . . . . 57

5.1 A Structured mesh being distorted by $\gamma$ radians . . . . . . . . . . . . . . 64

5.2 Error plots for initial stages of boundary layer development for three different distortion values while varying the order of the interpolation polynomial $"^{\mathrm{p}} \ldots \ldots \ldots \ldots \ldots \ldots \ldots \ldots$

5.3 Plots showing how the condition number increases with increase in mesh distortion, keeping the interpolation order "p" constant, such that $\gamma$ is measured in radians . . . . . . . . . . . . . . . . . . . . 72 
5.4 Plots of the error norm for different values of $\gamma$, keeping $p$ constant and varying the number of elements $h$, for $\tau=0.2$, where $\gamma$ is given in radians . 73

5.5 A combined Plot of the error norm for different values of $p$, while varying $\gamma$, for $\tau=0.2$ and $0.9 \ldots \ldots \ldots \ldots \ldots \ldots \ldots$

5.6 Plot showing the effect of $p$-refinement for $\tau=0.2 \ldots \ldots$. . . . 75

5.8 A color-map representing mesh quality using the Quadrilateral shape metric for two meshes with the same number of elements but increasing distortion $\quad 80$

5.11 A tri-quadrilateral Mesh for a Square plate along with a color-Map for qaulity using the Equi-anle Skew quality Metric . . . . . . . . . . . . . 83

5.12 A wire frame showing the unstructured meshing of a rectangular domain with a cylinder in between . . . . . . . . . . . . . . . . 84

5.13 A color-map for the quality of a tri-quadrilateral mesh for a rectangular domain with a cylindrical region in between . . . . . . . . . . . . . 85

5.14 A triangle displaying the variables used in the above equation . . . . . . . . 87

5.16 A color-map and a histogram representing mesh quality using the Equiangle skew quality metric for denser mesh than used for the square plate, with a circular hole at one end, and using the centroid as the Steiner point for quadrilateralization . . . . . . . . . . . . . . . 89

5.17 A color-map and a histogram as in the previous figure for the same geometry as above but with the Fermat point being used as the Steiner point to achieve the quadrilateralization . . . . . . . . . . . . . . 90 
5.18 A color-map and a histogram representing mesh quality using the Equiangle skew quality metric for an ever denser mesh and one edge rounded, using the centroid for quadrilateralization . . . . . . . . . . . . . . 91

5.19 A color-map and a histogram representing mesh quality using the Equiangle skew quality metric for the same geometry but using the Fermat point for quadrilateralization . . . . . . . . . . . . . . . . . 92 


\section{List of Tables}

5.1 Quality metrics used for a 2-D distorted element . . . . . . . . . . . . . 77 


\section{List of Definitions}

$$
\begin{aligned}
& \rho=\text { Density of fluid } \\
& \mathbf{u}=\text { Velocity field } \\
& \boldsymbol{\sigma}=\text { Stress tensor } \\
& \mathrm{g} \text { = Acceleration due to gravity } \\
& p=\text { Pressure } \\
& T=\text { Temperature } \\
& k=\text { Conductivity } \\
& \nu=\text { Kinematic viscosity } \\
& \hat{U}=\text { Internal energy per unit mass } \\
& \Phi=\text { Viscous dissipation function } \\
& C_{v}=\text { Specific heat at constant volume } \\
& \Omega=\text { Control volume } \\
& S=\text { Boundary surface } \\
& \text { b = Velocity at the boundary } \\
& \phi=\text { Velocity potential } \\
& \psi=\text { Stream function } \\
& \boldsymbol{\omega}=\text { Vorticity } \\
& \tau=\text { Unit vector tangential to the boundary } \\
& \mathbf{n}=\text { Unit vector normal to the boundary }
\end{aligned}
$$


$\mathbf{H}=$ Array of interpolation functions

B = Array of derivatives of interpolation functions

$N G L=$ Number of nodes of the Gauss-Lobatto points, $N_{G L}=p+1$

$p=$ Order of the interpolating polynomial

$\mathbf{U}^{e}=$ Elemental array of nodal velocity values

$\boldsymbol{\omega}^{e}=$ Elemental array of nodal vorticity values

$\mathbf{H}_{\omega}=$ Array of vorticity interpolation functions

$\mathbf{B}_{\omega}=$ Array of derivatives of vorticity interpolation functions

$\gamma=$ Angle of distortion in the controlled distortion experiment

$N^{*}=(N G L-1) * N_{e l}$

$N_{e l}=$ number of elements in the mesh along the vertical or horizontal direction for the square plate problem 


\section{Abstract}

The numerical solution of the incompressible Navier-Stokes equations offers an alternative to experimental analysis of fluid-structure interaction (FSI). We would save a lot of time and effort and help cut back on costs, if we are able to accurately model systems by these numerical solutions. These advantages are even more obvious when considering huge structures like bridges, high rise buildings or even wind turbine blades with diameters as large as 200 meters. The modeling of such processes, however, involves complex multiphysics problems along with complex geometries. This thesis focuses on a novel vorticity-velocity formulation called the Kinematic Laplacian Equation (KLE) to solve the incompressible Navier-stokes equations for such FSI problems. This scheme allows for the implementation of robust adaptive ordinary differential equations (ODE) time integration schemes, allowing us to tackle each problem as a separate module.

The current algortihm for the KLE uses an unstructured quadrilateral mesh, formed by dividing each triangle of an unstructured triangular mesh into three quadrilaterals for spatial discretization. This research deals with determining a suitable measure of mesh quality based on the physics of the problems being tackled. This is followed by exploring methods to improve the quality of quadrilateral elements obtained from the triangles and thereby improving the overall mesh quality. A series of numerical experiments were designed and

conducted for this purpose and the results obtained were tested on different geometries with varying degrees of mesh density. 


\section{Chapter 1}

\section{Introduction}

The flow of fluids can be seen all around us, be it the natural environment or almost any kind of technical field. Meteorological phenomena, combustion processes, HVAC systems, pollution, and the numerous processes in the human body are some examples of fluid flow we encounter almost everyday. The number of applications of fluid flow analysis is enormous: breathing, blood flow, turbines, airplanes, ships, windmills, and engines to name a few, making the analysis of flow one of the most important areas of research in the last half century.

Mathematically, the flow of fluids is represented by a system of non-linear partial differential equations known as the Navier Stokes Equations. These equations represent the conservation laws for mass, momentum, and energy for the concerned flow. The Navier- 
Stokes equations present a challenging problem to mathematicians and engineers in finding solutions related to proof of existence and finding accurate yet fast numerical methods with proper Boundary conditions.

The numerical Solution of the Navier-Stokes Equations is an important area of study in modern day engineering. It can be used to model numerous things of economic as well as academic interest. It offers an attractive alternative to the expensive, and sometimes extremely difficult to implement, experimental analysis of flow patterns.

\subsection{Fluid structure interaction}

One of the most important applications of the numerical solutions of these equations would be the numerical modeling of fluid-structure interaction (FSI). The non-linear dynamics involved in such interactions provides insights into numerous engineering problems such as the response of high rise buildings and bridges to strong winds, blood flow through arteries, vibrations in turbine blades, aerodynamic response characteristics of aircraft wings, areas of marine hydrodynamics which includes modeling fluid flow interaction with marine systems, offshore and coastal structures, underwater systems and structures, and the ever popular aerodynamic modeling of automobiles. These phenomena manifest themselves at a wide range of scales and present excellent opportunities for scientific discovery with a richness of technical application. 
An experimental analysis for some of the FSI problems might not always be the most attractive prospect. The case of a wind turbine is a perfect example to support the above statement, owing to the enormity of the surfaces involved. The economy of scales factors have driven companies to consider rotors with diameters going as large as 200 meters. Extrapolating experimental data from wind tunnels in such cases make things very complicated. Another interesting example would be the modeling of bridges and high rise buildings and their interaction with high winds. Such huge structures run the risk of stress related failures due to FSI, and hence require a very accurate modeling of flow patterns.

On the other extreme, problems of placing sensors on small-scale mechanisms with complex roto-translational motion, like the Micro-Air-Vehicle modelled on insect flight, makes an experimental analysis extremely difficult. A numerical scheme seems like an attractive alternative to experimental prototypes and should also help bring down the overall cost drastically. But a numerical solution comes with its own set of problems starting with complex physics involved in slender-body aeroelastic dynamics. The aeroelastic dynamics in slender bodies depends not only on the characteristic modes of the body structure itself, but also on the frequency and amplitude of the fluctuating aerodynamic forces. These forces are strongly affected by the dynamics of the vortex-wake shed from the body, which itself depends on the body's oscillations. Vortex-induced vibration can lead to catastrophic failure of engineering systems, as was clearly illustrated by the Tacoma Narrows Bridge disaster. Essentially, a periodic vibration ensues if the work done by the fluid on the body over a cycle is positive. This net energy transfer is significantly influenced by the phase 
of the induced side force relative to the body motion, which in turn is associated with the timing of the vortex dynamics (1).

A significant challenge in analyzing these systems is the fact that an oscillating body can produce a vortex wake that is very different from the classic Karman vortex street, which would translate into a complex fluctuating aerodynamic force. Many rototranslational mechanisms have a dynamic control system reacting to structural responses to fluid flow as well as varying loads, thereby optimizing efficiency and extending their lifetime. This control system would also have to be incorporated into the numerical model for an accurate representation. The problem is no longer just an unsteady flow simulation, but a multiphysics problem invloving non-linear structural dynamics as well as a dynamic control system in addition to the fluid flow model. All of this combined with the discretization of complex geometries makes it quite difficult to numerically solve the non-linear partial differential equations (PDEs) involved. Further complications may arise with time-marching integration of multiphysics problems. Adaptive variable-timestep/variable-order ODE algorithms provide a way to improve the efficiency of time-marching schemes. But finding a way to combine those adaptive algorithms with the discretization of the spatial PDE problem has proved to be difficult. An innovative computational scheme to solve these problems was introduced in (2).This scheme is called the Kinematic Laplacian Equation (KLE) method. The KLE invloves a hybrid formulation of the Navier-Stokes equations using velocity and vorticity as the primary variables, rather than the conventional formulation in terms of pressure and velocity, and is a natural extension of the well-established vorticity-stream 
function methods. The emergence of vorticity-velocity methods might be considered one of the most recent innovations in the computational solution of time-dependent viscous flows. Even though the appearance of what could be regarded as the first vorticity-velocity approach may be traced as early as 1976 (3), it is only during the last decade or so that a systematic research effort has been applied to the development of this family of methods (see see $(2,4)$ for a complete list of references). The vorticity-velocity methods present several advantages compared with the classical formulation on primitive variables (velocitypressure) or with their vorticity-stream-function cousins. This is discussed in greater detail in Chapter 2.

The KLE algorithm solves the vorticity transport equation as an ODE problem in time with input velocity from the solution of a modified Poisson's equation in velocity, called the Kinematic Laplacian Equation, at each spatial node. The input to solve the KLE is provided by the time integration of the vorticity at each time step. Thus, it creates an evolving scheme in which the KLE provides the input for the ODE algorithm and vice-versa. Since time is the only iteration variable present it is now possible to couple the fluid analysis with other physical mechanisms (e.g. structural response, control-system dynamics, etc.) by adding more equations to the ODE system. The KLE also shows a substantial tolerance to the use of unstructured meshes, which allows a more suitable meshing of complex geometries than structured-mesh approaches would permit. The latter is a very convenient feature for dealing with the complex aerodynamic shape of wind-turbine blades, helicopter-rotor blades, insect wings, or other aerodynamic surfaces. 


\subsection{An emerging field of application: The wind power chal-}

\section{lenge}

This research is a small yet important part of the ongoing work towards the advancement of computational mathematical models for complex multiphysics problems involving fluidstructure-control interaction that are present in many engineering designs, providing a fundamental tool for a better understanding of the underlying physics. One such important

engineering problem is the harnessing of wind power. Given the current trend of looking towards cheaper and cleaner ways to meet the ever increasing energy demand, wind energy might just be the answer to our growing needs. It is not only getting cheaper with technological innovations to harness wind energy on a large scale, wind power is also one of the cleanest ways to produce energy. During the last three decades there has been a spontaneous tendency in the wind-turbine industry to increase the size of the state-of-theart machine (6) and substantially reduce the cost of wind energy. Output power of these turbines range from 3.6 to $6 \mathrm{MW}$, with rotor diameters up to 127 meters (Figure 1.1). Nextgeneration offshore turbines with rotor diameters up to 200 meters have been suggested (7). The technological challenge in wind power nowadays is to develop a next generation of upscaled low-cost turbines that may further reduce generation costs. If this generation of superturbines is successfully developed, wind-energy costs would be reduced substantially. In fact, in favorable sites, it might be feasible to produce hydrogen as an alternative fuel 


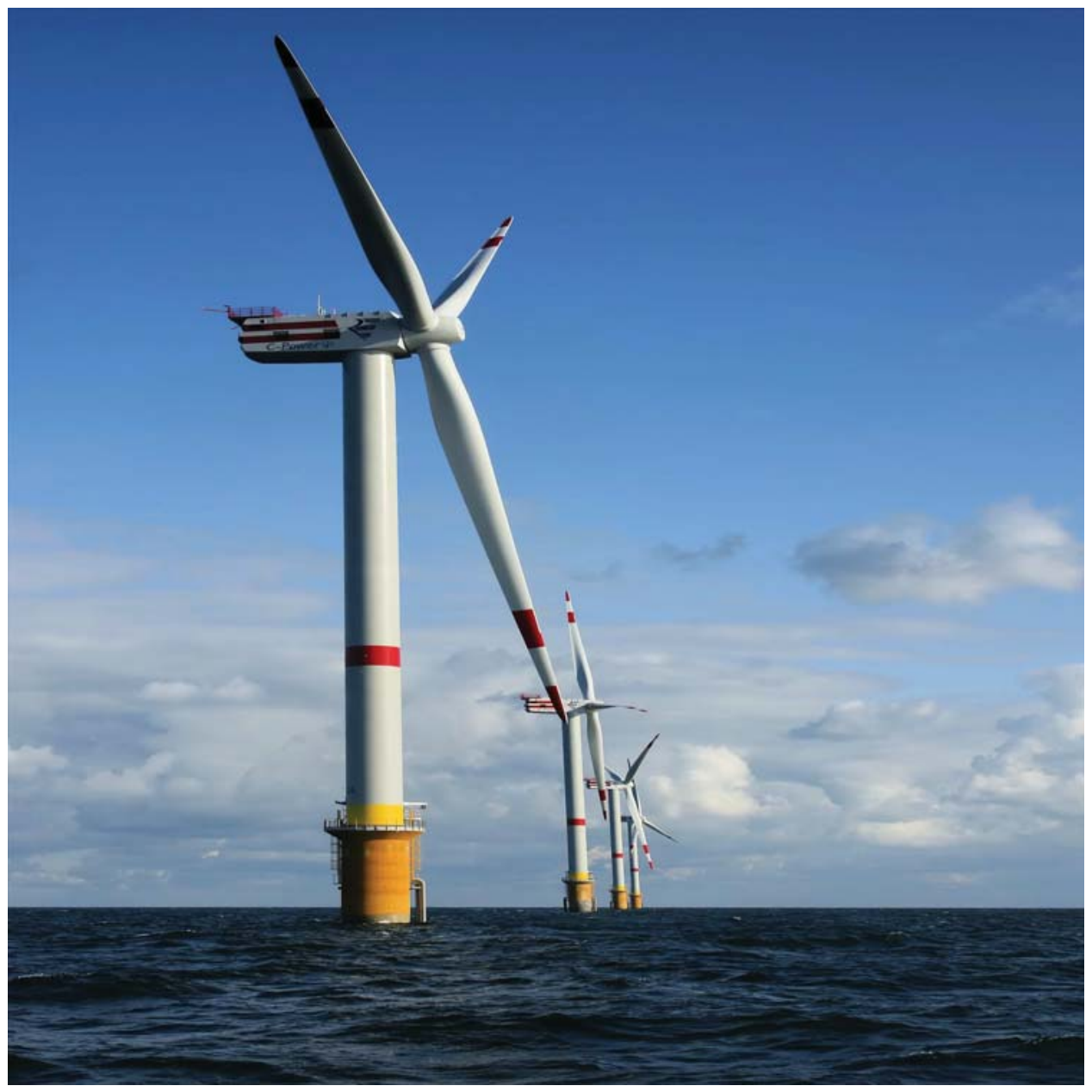

Figure 1.1: The REpower M5 5-megawatt turbine, with a rotor diameter of approximately 126 meters (from (5)).Permission to reuse in Appendix C.

in competitive terms, thereby getting rid of a significant roadblock towards developing hydrogen as an alternative fuel. Current wind-turbine blade technology based on composite laminates is labor-intensive and requires a highly-qualified workforce, creating a critical bottleneck in terms of industrial workforce and infrastructure that hampers a rapid expansion of wind-energy. It also poses a barrier to turbine upscaling by increasing the cost of the rotor as turbine size increases. The structural conception of today's blades also poses huge challenges in terms of transport logistics and crane capacity. Transportation cost in- 
creases as blades grow in length. The risk of damage during transportation, and hence, the cost of insurance, also increases with length. Moreover, while the rest of the turbine subsystems may be treated as modules assembled on site, blades are one-piece monolithic components, substantially complicating transport logistics. Limitation in crane capacity is the other critical factor to take into account during the turbine assembly phase. Thus, transport and lifting logistics may impose a premature limit for turbine upscaling, even before the actual limits in blade length for the current manufacturing technology are reached. Blades operate under a complex combination of fluctuating loads, and huge size differences complicate extrapolation of experimental data from the wind-tunnel to the prototype scale. Hence, computer models of fluid-structure interaction phenomena are particularly relevant to the design and optimization of wind-turbines. The wind-turbine industry is increasingly using computer models for blade structural design and for the optimization of its aerodynamics. But the complex interaction of physical processes that characterize the coupled aeroelastic problem still exceeds the capacities of existing commercial simulation codes. The result is an industry to be cautious with the introduction of new concepts in order to ensure reliability. Innovations are likely to introduce changes in structural response and may possibly require different control strategies, which should be taken into account if the development of a new prototype blade is considered. Research efforts within the established parameters of the composite-laminate monolithic blade concept would not produce the breakthrough that is needed in wind-power evolution. A better understanding of the underlying physics is needed in order to introduce innovative concepts like modular blades 
and improved control strategies. This is where the KLE plays a pivotal role owing to its ability to create a common framework for modular integration of the aeroelastic model with the control system dynamics.

\subsection{Thesis outline}

The focus of the current thesis is to improve the efficiency of the spatial discretization of an incompressible viscous flow problem using the KLE method. The problem considered here is the semi-infinite region of stationary fluid bounded by an infinite horizontal plate and given a sudden velocity in its own plane and thereafter, maintained at that speed. This problem, along with its analytical solution, is discussed further in context with the KLE in Chapter 4. Since the unstructured meshes are better suited to complex geometries than structured meshes, their use for implementing the KLE is studied.

This thesis is divided into two parts. The first part is concerned with carrying out controlled distortions of a structured mesh and using the distorted meshes obtained to solve the abovementioned flow problem using the KLE. The results are then analyzed to select a mesh quality metric best suited for the KLE method. The criteria for assessing an element's quality should be problem specific to some extent. This metric is then used to come up with a possible change in the kind of unstructured meshes used. This change constitutes the second part of the thesis. This part is concerned with modifying the methodology used 
to transform an unstructured triangular mesh into a quadrilateral one. The end result is then compared with the previous quadrilateralization using the quality metric decided upon in the first part. The mesh quality is analyzed for two dimensional geometries ranging from a square region to a few aerofoils. The results obtained are quite promising and present a strong case in favor of this approach in regard to both quality of the mesh obtained as well as the computational effort required for the quadrilateralization.

As mentioned before, the motivation for this research is the analysis of flow over wind turbine blades. Since this type of flow can be assumed to approximate incompressible flow, that is exactly the kind of flow which will be considered for this thesis. The next Chapter deals with an introduction to the incompressible Navier-Stokes equations and gives a breif outline of some of the more popular solution methods in use. Chapter 3 is concerned with the hybrid methods based on a vorticity/velocity approach to solve the Navier-Stokes equations. The Chapter then goes on to introduce the KLE along with its variational formulation. Chapter 4 deals with the numerical implementation of the KLE. Chapter 5 explains the controlled distortion experiment and is concerned with the analysis of the results obtained by the KLE based on the experiment. This Chapter is also concerned with the various quality parameters analyzed and the subsequent quadrilateralization method developed for the triangular mesh. Chapter 6 summarizes the results and ends with a brief on future prospects. 


\section{Chapter 2}

\section{Numerical methods}

\subsection{The Navier-Stokes equations}

Navier-Stokes equations is a system of non-linear Partial differential Equations of the second order. These consist of three basic conservation equations

1. Conservation of mass or the continuity equation

$$
\frac{\partial \rho}{\partial t}+\nabla \cdot(\rho \mathbf{u})=0
$$

where $\rho$ is the density, $\mathbf{u}$ is the velocity field and $t$ is the time.

2. Conservation of momentum

$$
\rho \frac{\mathrm{d} \mathbf{u}}{\mathrm{d} t}=\rho \mathbf{g}-\nabla P+\nabla \cdot \boldsymbol{\sigma}
$$


where, $P$ is the pressure, $\boldsymbol{\sigma}$ is the stress tensor and $\rho \mathbf{g}$ is the body force.

\section{Conservation of energy}

$\rho \frac{\mathrm{d} \hat{u}}{\mathrm{~d} t}+p(\nabla \cdot \mathbf{u})=\boldsymbol{\nabla} \cdot(k \nabla T)+\boldsymbol{\Phi}$

where $\hat{U}$ is the internal energy per unit mass, $\mathrm{k}$ is the thermal conductivity, $\nu$ is the kinematic viscosity and the function $\Phi$ represents energy dissipated due to viscous effects.

This set of equations may completely define fluid motion allowing us to solve for various flow quantities like pressure, velocity, temperature, and density. In the more general case of the incompressible flow of Newtonian fluids, the density is considered constant and the above equations reduce to

1. Conservation of mass or the continuity equation

$$
\nabla \cdot \mathbf{u}=0
$$

2. Conservation of momentum

$$
\rho \frac{\mathrm{d} \mathbf{u}}{\mathrm{d} t}=\rho g-\nabla P+\mu \nabla^{2} \mathbf{u}
$$

where $\mu$ is the coefficient of dynamic viscosity.

3. Conservation of energy

$$
\rho C_{v} \frac{\mathrm{d} T}{\mathrm{~d} t}=k \nabla^{2} T+\Phi
$$

where $C_{v}$ is the specific heat at constant volume. 


\subsection{Numerical Solution of the incompressible viscous flow}

The following section deals with some of the more popular formulations of the Navier-

Stokes equations modeling a viscous incompressible flow of a homogeneous fluid in an inertial frame of reference, along with some discussion on the issues of boundary conditions. As mentioned before, for a homogeneous incompressible flow, the density remains constant giving us a set of equations (2.1), (2.2), (2.3). These equations clearly imply the decoupling of the momentum equation with the energy equation for the incompressible flow. The most obvious advantage of this decoupling is that the momentum equation and the continuity equation can now be solved independent of temperature to obtain the required velocity and pressure field. This decoupling might seem like it makes things easier, but that is not the whole picture, as will become clear in the subsequent discussion.

The first step in finding a numerical solution to the Navier-Stokes equations would be to decide upon the set of variables representing the equations followed by a set of boundary conditions. The two main formulations of the Navier-Stokes equations are the primitive variable formulations and the non-primitive variable formulations. Both have their own set of advantages and disadvantages, which shall be discussed in brief, but in both cases the major problem and the oldest point of contention are the boundary conditions. It is mostly the pressure and vorticity which pose a problem since they do not have what can be called an obvious physical representation at the boundaries. 


\subsubsection{Primitive variables}

The most fundamental formulation of the incompressible Navier-Stokes equations would be the pressure-velocity based formulations. These are what are called the primitive variable formulations. The Navier-Stokes equations for incompressible viscous flow in terms of the primitive variables can be written as

$$
\begin{array}{r}
\frac{\partial \mathbf{u}}{\partial t}+u \cdot \nabla \mathbf{u}=-\nabla p+\nu \nabla^{2} \mathbf{u}+\mathbf{g} \\
\boldsymbol{\nabla} \cdot \mathbf{u}=0
\end{array}
$$

where, $p=P / \rho$ defined in a spatial domain $\Omega$ with $S$ as its boundary. Here $\mathbf{u}$ is the velocity, $p$ is the pressure divided by the fluid density (constant), and $\nu$ is the kinematic viscosity. The Navier-Stokes equations represented by the primitive variables can be called a mixed elliptic parabolic equation being parabolic in time mainly because of the convective diffusive term and elliptic in space owing to the interaction between pressure and the continuity equation. To define the above problem completely, the equation needs to be supplemented by a set of boundary conditions specifying it as an initial value boundary value (IVBV) problem. The most common approach would be to start with the specification of an initial value for the velocity in $\Omega$

$$
\mathbf{u}\left(x, t_{0}\right)=\mathbf{u}_{0}(x) \text {, such that } \boldsymbol{\nabla} \cdot \mathbf{u}_{0}=0 \text {, where } x \in \Omega
$$

followed by specifying the velocity at the boundary

$$
\mathbf{u}(x, t)=\mathbf{u}_{S}(x, t), x \in S=\mathbf{b}
$$


along with a global continuity condition obtained by integrating the continuity equation over the entire volume and then using the Gauss theorem

$$
\oint_{S} \mathbf{n} \cdot \mathbf{b d} \mathbf{S}=\mathbf{0}
$$

where, $\mathbf{n}$ is a unit vector normal to the boundary surface. So in this simplest of representations there is indeed no boundary condition for pressure, giving rise to a series of problems which are still a topic of debate.

\section{Solution strategies}

As discussed in the previous section, the main difficulty in the numerical solution of the incompressible Navier-Stokes equations comes in the form of understanding pressure. Unlike velocity, pressure does not have any explicit representation. There is no evolutionary pressure equation. The main reason is that since we have assumed an incompressible condition, pressure now loses its thermodynamic meaning and serves only as some kind of a Lagrange operator to ensure the incompressibility condition. Since the pressure term is of elliptic character, the solution anywhere would be influenced by the solution at every point in the domain. Physically this means that the pressure waves travel at infinite velocity to all points in the domain for every time step. In other words, the pressure has to adjust instantaneously throughout the domain to account for any disturbance in order to maintain zero divergence of velocity. This leads to the intricate coupling between the two variables. This statement is the driving factor for non-fractional approach or, in other words, the standard discretization approach. Thus a numerical scheme with proper boundary conditions must be devised which can take into consideration the implicit coupling between pressure and 
velocity and keeps the flow incompressible throughout the domain. At the same time, the method should be able to decouple the pressure and velocity and solve for each separately to avoid having to tackle the unwieldy simultaneous equations.

It is, in fact, possible to solve this set of equations simultaneously as a coupled system (8). This is rarely seen in finite difference methods (8) due to the complex matrices obtained as opposed to a regular block diagonal matrix. Though this method is more common in finite element analysis, it does have its own set of difficulties, as mentioned by (9) arising from :

1. The equations are non-linear.

2. The continuity equation.

3. The coupling between the set of equations owing to the advection term and continuity equation.

The most common approach to getting rid of the continuity equation is Poisson's pressure equation obtained by taking the divergence of the momentum equation and using the continuity condition. This, along with other details, will be discussed in the subsequent section.

The two main approaches in a pressure-velocity formulation are the fractional step and the non-fractional step methods. 


\subsubsection{Non-fractional step methods}

The major difference between the two approaches is that in non-fractional step methods the velocity and pressure evolve simultaneously, whereas in the fractional step method the convection part is treated separately from the pressure or incompressibility part. The equations (2.4) and (2.5) can be linearized by discretizing in time and neglecting the body forces to give

$$
\begin{gathered}
\frac{\mathbf{u}^{n+1}-\mathbf{u}^{n}}{\triangle t}+\nabla p^{n+1}=\nu \nabla^{2} \mathbf{u}^{n+1}-\mathbf{u}^{n} \cdot \nabla \mathbf{u}^{n} \\
\nabla \cdot \mathbf{u}^{n+1}=0
\end{gathered}
$$

where, $\mathbf{u}$ is the velocity vector and $p$ is the pressure term equation (2.9) can be written as

$$
[-\triangle+\gamma \mathbf{I}] \mathbf{u}^{n+1}+\nabla p^{n+1}=g\left(\mathbf{u}^{n}\right)
$$

where, $\nu$ is the kinematic viscosity, $\mathbf{I}$ is an Identity matrix, $\gamma=\frac{1}{\nu \triangle t}$ and $g\left(\mathbf{u}^{n}\right)=\gamma \mathbf{u}^{n}-$ $\nu^{-1} \mathbf{u}^{n} \cdot \nabla \mathbf{u}^{n}$

Here, a two-level scheme has been adopted, treating the pressure and viscous term implicitly and the nonlinear advection term explicitly in time. There can be other methods in time discretization such as a fully implicit scheme or even a semi-implicit scheme using linearization techniques. A popular method is to use a higher order explicit scheme like the Adams-Bashforth scheme for the advection term and a Crank-Nicolson scheme for the linear terms. This can be followed by linearizing the advection term using a point iteration 
scheme. There are many other higher order and more accurate schemes, but the one mentioned in the above equations is just for explanatory purposes. As previously mentioned, a Poisson's Equation is obtained by taking the divergence of the momentum equation and using the continuity condition. This gives us the following set of equations :

$$
\begin{array}{r}
{[-\Delta+\gamma \mathbf{I}] \boldsymbol{\nabla} \cdot \mathbf{u}^{n+1}+\boldsymbol{\nabla} p^{n+1}=g\left(\mathbf{u}^{n}\right)} \\
\boldsymbol{\nabla}^{2} p^{n+1}=\boldsymbol{\nabla} \cdot g\left(\mathbf{u}^{n}\right)
\end{array}
$$

Even though the above relation is obtained by the continuity equation, its solution does not imply the incompressibility condition, all it shows is that $[-\Delta+\gamma \mathbf{I}] \nabla \cdot \mathbf{u}^{n+1}=0$. Therefore it simply tells us that $\boldsymbol{\nabla} \cdot \mathbf{u}^{n+1}$ is harmonic but not necessarily zero. However, Kleiser and Schumann (10) showed that if divergence at the boundary is forced to zero

$$
\left.\boldsymbol{\nabla} \cdot \mathbf{u}^{n+1}\right|_{S}=0
$$

incompressibility should be ensured throughout the domain. This follows from the mean value theorem/ extremum theorems of harmonic functions which means if some harmonic function equals a constant at the surface completely enclosing a domain, its value will be equal to that constant throughout the domain.This leads us to a BVP with two elliptic equations

$$
\begin{array}{r}
{[-\triangle+\gamma \mathbf{I}] \nabla \cdot \mathbf{u}^{n+1}+\nabla p^{n+1}=g\left(\mathbf{u}^{n}\right)} \\
\nabla^{2} p^{n+1}=\boldsymbol{\nabla} \cdot g\left(\mathbf{u}^{n}\right) \\
\left.\mathbf{u}^{n+1}\right|_{S}=\mathbf{b}^{n+1} \\
\left.\boldsymbol{\nabla} \cdot \mathbf{u}^{n+1}\right|_{S}=0
\end{array}
$$


along with the global constraint (2.8) i.e. $\oint_{S} \mathbf{n} \cdot \mathbf{u}_{\mathbf{S}} \mathbf{d} \mathbf{S}=\mathbf{0}$. Here there are two boundary conditions for $\mathbf{u}$ and none for pressure, which means that the above equations still have to be solved simultaneously. So, it is imperative to look for some method to decouple the two equations by coming up with a legitimate boundary condition for pressure. As mentioned in (11), Glowinksi and Pironneau devised a Finite Element method based on an additional equation for the scalar velocity potential. Another method was based on the influence matrix technique proposed by Kleiser and Schumann (11). Both these methods propose to implement the pressure boundary conditions using an additional linear problem. This was followed by Quartapelle and Napolitano’s paper on implementing integral Boundary conditions on pressure (11) which seemed to provide a better physical interpretation owing to the elliptic nature of the Poisson's equation for pressure. A brief introduction to these methods can be found in Appendix A.

\subsubsection{Fractional step methods}

This method, first introduced by Chorin and Tenman, is one of the most widely used methods for solving the primitive variable formulation of the Navier-Stokes equations. Consider the following form of the Navier-Stokes equations, with the body forces neglected

$$
\begin{gathered}
\frac{\partial \mathbf{u}}{\partial t}+u \cdot \boldsymbol{\nabla} \mathbf{u}=-\boldsymbol{\nabla} p+\nu \boldsymbol{\nabla}^{2} \mathbf{u} \\
\boldsymbol{\nabla} \cdot \mathbf{u}=\left.0 \quad \mathbf{u}\right|_{S}=\mathbf{b}
\end{gathered}
$$


The general procedure is to advance the velocity in time and approximate an intermediate velocity from the first equation, without considering the pressure term. As already mentioned, the pressure term in the above equation acts as a Lagrange multiplier to enforce incompressibilty at every time-step, therefore, the calculated velocity will not be divergence free. This velocity is then used to solve an elliptic equation to obtain a divergence free velocity by enforcing the incompressibilty condition. Subsequently a pressure field is determined for that time step. In the intermediate step, the following equation is obtained by avoiding completely the pressure term

$$
\frac{\mathbf{u}^{*}-\mathbf{u}^{n}}{\triangle t}=-\left(\mathbf{u}^{n} \cdot \nabla\right) \mathbf{u}^{n}+\left.\nu \nabla^{2} \mathbf{u}^{n} \quad \mathbf{u}^{*}\right|_{S}=\mathbf{b}^{n+1}
$$

Obviously, as stated above, the velocity field $\mathrm{u}^{*}$ would not be divergence free, which leads to the next step

$$
\begin{array}{r}
\frac{\mathbf{u}^{n+1}-\mathbf{u}^{*}}{\triangle t}=-\nabla p^{n+1} \\
\boldsymbol{\nabla} \cdot u^{n+1}=0 \\
\left.\mathbf{n} \cdot \mathbf{u}^{\mathbf{n}+\mathbf{1}}\right|_{\mathbf{S}}=\mathbf{n} \cdot \mathbf{b}^{\mathbf{n}+\mathbf{1}}
\end{array}
$$

Equation (2.18) can also be formulated as

$$
\mathbf{u}^{*}=\mathbf{u}^{n+1}+\triangle t \nabla p^{n+1}
$$

where, $\nabla p^{n+1}$ is not the gradient of pressure but of some artificial scalar function proportional to the unknown Pressure often referred to as the "Pseudo-pressure". The quantity 
$\mathbf{u}^{n+1}$ which is required the velocity field is actually the solenoidal component of $\mathbf{u}^{*}$ and not the real $\mathbf{u}^{n+1}$ as the tangential boundary condition is not necessarily met. The normal boundary value for velocity is a consequence of the above step being inviscid. So the required velocity is calculated by projecting the velocity $\mathbf{u}^{*}$ onto a solenoidal space. The basis for the above step is the helmholtz-hodge decomposition of the velocity field (due to Ladyzhenskaya) which states that any vector field can be decomposed as $\mathbf{v}=\mathbf{w}+\boldsymbol{\nabla} \phi$ where, $\mathbf{w}$ is solenoidal and $\mathbf{n} \cdot \mathbf{w}=\mathbf{0}$ and $\phi$ is the potential function with its gradient giving the irrotational component of $\mathbf{v}$. The gradient term can be further decomposed into $\boldsymbol{\nabla} \phi=\boldsymbol{\nabla} \phi_{0}+\nabla h$, where $h$ is a harmonic function and $\left.\phi_{0}\right|_{S}=0$. This leads to the vector $\mathbf{v}$ being decomposed into

$$
\mathbf{v}=\mathbf{w}+\boldsymbol{\nabla} \phi_{0}+\nabla h
$$

Using this decompostion for the divergent $\mathbf{u}^{*}$

$$
\mathbf{u}^{*}=\mathbf{w}+\nabla \phi_{0}+\nabla h
$$

Adding and subtracting another harmonic function $h_{B}$ to the RHS of equation (2.23)

$$
\left.\mathbf{u}^{*}=\left[\mathbf{w}+\boldsymbol{\nabla} h_{B}\right]+\left[\boldsymbol{\nabla}\left(h-h_{B}\right)\right]+\boldsymbol{\nabla} \phi_{0}\right] \quad \mathbf{n} \cdot \boldsymbol{\nabla} \mathbf{h}_{\mathbf{B}}=\mathbf{n} \cdot \mathbf{b}
$$

Clearly $\left[\mathbf{w}+\nabla h_{B}\right]$ represents $\mathbf{u}^{n+1}$ for an incompressible flow not satisfying the no-slip condition for the second half step. Thus, $\mathbf{u}^{n+1}$ can be found using a projection of $\mathbf{u}^{*}$ on the divergence free space. 
In order to calculate the velocity from the equations (2.18) and (2.20) the divergence of (2.18) is substituted into (2.19) to get the Poisson's Equation for pressure also callled the PPE

$$
-\nabla^{2} p^{n+1}=\frac{-1}{\triangle t} \nabla \cdot \mathbf{u}^{*}
$$

Using equation (2.20) along with the boundary condition $\left.\mathbf{u}^{*}\right|_{S}=\mathbf{b}^{n+1}$ the following boundary condition for the PPE can be derived

$$
\left.\mathbf{n} \cdot \nabla \mathbf{p}^{\mathbf{n}+\mathbf{1}}\right|_{\mathbf{S}}=\mathbf{0}
$$

Once the pressure field is determined, the required velocity field can be determined from equations (2.18) and (2.20). The basic disadvantage here as mentioned before is that the second half step ensuring the incompressibility condition is inviscid, thereby, able to ensure only the normal component of the velocity boundary condition. This error is slightly qualified owing to the fact that the velocity boundary condition in the first half step is the no-slip condition. There are methods that introduce the viscous component in the second half step. One such method is mentioned in (10) in the form of a Crank-Nicholson scheme

$$
\begin{array}{lrl}
\frac{\mathbf{u}^{*}-\mathbf{u}^{n+1}}{\triangle t} & =-\left(\mathbf{u}^{n} \cdot \nabla\right) \mathbf{u}^{n}+\frac{1}{2} \nu \nabla^{2} \mathbf{u}^{n} & \left.\mathbf{u}^{*}\right|_{S}=\mathbf{b}^{n+1} \\
\frac{\mathbf{u}^{n+1}-\mathbf{u}^{*}}{\triangle t}=-\nabla p^{n+1}+\frac{1}{2} \nu \nabla^{2} \mathbf{u}^{n+1} & \left.\mathbf{u}^{n+1}\right|_{S}=\mathbf{b}^{n+1}
\end{array}
$$

This is second order accurate for the viscous term but, like the non-fractional step schemes, some complicated pressure condition must be used to enforce the incompressibility. 


\subsubsection{Artificial incompressibility}

This method, first proposed by Chorin in 1967, allows analysts to take advantage of the immense advances made in the analysis of compressible flow. The main idea of this method is to replace the continuity equation with a slightly modified version in order to make it compressible and, hence, solve it as an evolution equation in pressure. In order to achieve that a time derivative of the pressure term is added to the continuity equation giving

$$
\frac{1}{\beta} \frac{\partial p}{\partial t}+\frac{\partial \mathbf{u}_{i}}{\partial \mathbf{x}_{i}}
$$

This was originally introduced for the steady state Navier-Stokes equations, so that the artificial compressibility term vanishes as the steady state is achieved. Here ' $t$ ' does not represent the real physical time but an artificial 'pseudo' time. This gives a mixed hyperbolic/parabolic form of equations, and many algorithms developed for similar compressible flows could be used to solve these equations. For a steady state formulation Chorin proposed a leap-frog time differencing scheme for pressure and a Dufort-Frankel space differencing method for velocity at the regular grid points. Peyret and Taylor adopted the staggered grid formulation explicit in time (12). What this method implies on a physical level is that pressure waves now travel at a finite speed depending on the incompressibility factor $\beta$ rather than instantaneously propagate to all points in the domain, thus the choice of $\beta$ is very important. The higher its value the closer the formulation will be to incompressible flow. But at the same time, however, too high a value will tend to make the equation stiff. But if $\beta$ is too small then the propagation speed will be too slow which will effect 
other factors like the viscous boundary layer, flow separation, etc. which might prevent convergence.

The governing equations are iterated in the 'pseudo' time until steady state is achieved. Although because of the compressibility introduced in the continuity equation this method was not preferred for unsteady flow, it has been proven successful for such flows as well (13). The general idea for unsteady flows would be to use an iterative procedure using an artificial compressibility method for each physical time step, ensuring that incompressibility is met at each step.

\subsubsection{Non-Primitive variables}

Given the importance of vorticity as a physical variable, especially in vortex dominated flows, a vorticity-based approach to formulate the Navier-Stokes equations is a popular alternative to the primitive variable formulation. In such cases, it makes more sense to analyze the flow based on vorticity, which has an extensively researched and understood transport equation. The study of vortex generation at boundaries, along with its diffusion and advection are very important in analyzing flow seperation, drag etc. in vortex dominated flows. Mathematically, owing to the vorticity $\boldsymbol{\omega}$ being one order higher than the velocity $\mathbf{u}$ a vorticity formulation implicitly gives a more accurate velocity field. Vorticitybased methods also give a better estimate of the skin friction since they are based more on 
the shearing process itself. For flows with high Reynold's number the vorticity seems to be concentrated in the wake region, thereby, greatly reducing the computational domain. However, this formulation is also plagued by its own set of problems. The kinematic problem for the vorticity-velocity relation is overdetermined as it has both the Neumann and Dirichlet boundary conditions prescribed, whereas the dynamic vorticity transport equation has no boundary conditions for vorticity. Also, there does not seem to be any physical boundary condition for the vorticity independant of the velocity boundary conditions.

The creation of vorticity can be attributed to the no slip boundary Condition which results in a torque and hence an angular velocity being imparted on the packets of fluid. This vorticity creation at the boundary should be represented by the vorticity boundary conditions and has been the general reasoning used to tackle this issue. Lighthill, One of the pioneers in this approach, proposed a fractional step method starting with an arbitrary vorticity boundary condition (14). This results in a velocity field which does not satisfy either of the two velocity conditions. He then proposed adding a velocity potential to take care of the normal flow, thereby getting slip velocity at the boundary. This slip velocity was called the vortex sheet and said to represent the boundary vorticity. Chorin (14) proposed a similar approach using the Prandtl boundary layer approximation. He basically split the NavierStokes equations into a viscid and an inviscid part. The Euler equation is then solved to give a slip velocity at the boundary. To get rid of this, vortex sheets are introduced and the resulting vorticity field is then used to solve the diffusion equation to get the correct vorticity field at the desired time step. This formulation does not seem to satisfy the no-slip 
as also the normal boundary condition for velocity simultaneously and independent of the geometry.

There have been many such models based on generation of vorticity at the boundary, but there is an entirely different school of thought which believes that a vorticity creation method does not fully explain vorticity interaction with solid boundaries. This led Quartapelle and Valgriz (10) to introduce an integral constraint on the vorticity. This 'nonlocal' approach couples the vorticity everywhere in the domain to the boundary velocity. A similar approach was adopted by Anderson (14) who suggested requiring the time derivative of these integral constraints be made to vanish. These methods as per Quartapelle (10) are the true representation of the vorticity diffusion and interaction with solid walls. These are ofcourse a kind of projection methods, where an initial 'wrong' vorticity, based on an arbitrary vorticty boundary value is 'corrected' by a projection onto the space of harmonic functions. This is achieved by the integral condition. This Chapter deals with a particular type of formulation called the non-primitive variable formulation and a brief overview of solution strategies based on Quartapelle's book (10) is given for both two and three dimensional flows. The next Chapter covers hybrid formulation, also called the vorticity-velocity formulation. 


\subsubsection{Vorticity-stream function formulation for two dimensional flows}

One possible way to circumvent the problem of pressure boundary conditions is to eliminate the pressure term entirely. This is exactly what is achieved in the vorticity-stream function formulation of the Navier-Stokes equations. In this formulation the Navier-Stokes equations are represented in terms of the vorticity $\boldsymbol{\omega}$ and the stream function $\psi$. So now the unknowns are $\boldsymbol{\omega}$ and $\boldsymbol{\psi}$ instead of $u, v, p$, reducing the number of unknowns by one. It also presents the added advantage of automatically taking care of the incompressibility condition owing to a property of the stream function. In two dimensions the above representation comprises two scalar equations obtained as follows:

In two dimensions vorticity $\boldsymbol{\omega}$ is a scalar given by

$$
\omega=\nabla \times \mathbf{u} \cdot \mathbf{k}
$$

while the velocity can be represented as the curl of a Stream-function $\psi$ given by

$$
\mathbf{u}=\nabla \times \psi
$$

Equation (2.31) clearly implies that $\boldsymbol{\nabla} \cdot \mathbf{u}=0$. Substituting equation (2.31) in (2.30) gives the Poisson's equation for the Stream-function

$$
-\nabla^{2} \psi=\omega
$$

Taking the curl of the momentum equation and using equations (2.30), (2.31) and $\boldsymbol{\nabla} \cdot \mathbf{u}=0$ gives the vorticity transport equation

$$
\frac{\partial \omega}{\partial t}+J(\omega, \psi)=\nu \nabla^{2} \omega
$$


where, $J(\omega, \psi)$ is the Jacobian matrix representing the curl of the advection term namely, $\nabla \times[(\mathbf{u} \cdot \nabla) \mathbf{u}]$

The Dirichlet and Neumann conditions for the above two equations are derived conditions deduced by separately tackling the normal and tangential components of boundary conditions of the velocity equation (2.7) i.e. $\left.\mathbf{u}\right|_{S}=\mathbf{b}$. They are given by, $\left.\psi\right|_{S}=a$ and $\left.\frac{\partial \psi}{\partial n}\right|_{S}=b$, where, $a=\int_{s_{1}}^{s} \mathbf{n} \cdot \mathbf{b d S}$ and $b=-\boldsymbol{\tau} \cdot \mathbf{b}$ given that $s_{1}$ is any fixed point on the boundary and $\tau$ is a unit vector tangential to the boundary. An initial condition for the vorticity can also be derived using the definition of vorticity and the initial condition for velocity equation (2.6) giving the following initial condition

$$
\left.\omega\right|_{t=0}=\left(\boldsymbol{\nabla} \times \mathbf{u}_{0}\right) \cdot \mathbf{k}
$$

Therefore the Navier-Stokes equations in the two dimensional vorticity-Stream function formulation can be written as

$$
\begin{array}{r}
\frac{\partial \omega}{\partial t}+J(\omega, \psi)-\nu \nabla^{2} \omega=0 \\
-\nabla^{2} \psi=\omega \\
\left.\psi\right|_{S}=a,\left.\quad \frac{\partial \psi}{\partial n}\right|_{S}=b \\
\left.\omega\right|_{t=0}=\left(\boldsymbol{\nabla} \times \mathbf{u}_{0}\right) \cdot \mathbf{k}
\end{array}
$$

Provided that

$$
\begin{array}{r}
\boldsymbol{\nabla} \cdot \mathbf{u}_{0}=0 \\
\frac{\partial a(S, 0)}{\partial s}=\mathbf{n} \cdot \mathbf{u}_{\mathbf{0}}
\end{array}
$$


One of the problems with this kind of formulation is the nonlinear advection term which also couples the vorticity and stream function variables. The other important issue is the overspecification of $\psi$ owing to both Dirichlet and Neumann conditions present as opposed to the underdetermined problem for $\omega$ with no boundary condition specified for it. The nonlinear terms can be dealt with using the standard linearizing techniques for nonlinear equations. Some of the ways in which the problem of the boundary conditions is tackled are discussed below.

\subsubsection{Biharmonic formulation}

One way to avoid the boundary value problem for vorticity is to eliminate the vorticity term from the transport equation. This is achieved by substituting the Poisson's equation for the stream function into the voticity transport equation resulting in the following equation

$$
\begin{array}{r}
\frac{\partial^{2} \boldsymbol{\nabla}^{2} \psi}{\partial t}-\nu \boldsymbol{\nabla}^{4} \psi+J\left(\boldsymbol{\nabla}^{2} \psi, \psi\right)=0 \\
\left.\psi\right|_{S}=a \quad,\left.\quad \frac{\partial \psi}{\partial n}\right|_{S}=b
\end{array}
$$

where, $\psi_{0}$ is the solution of the Dirichlet problem

$$
-\boldsymbol{\nabla}^{2} \psi_{0}=\left(\boldsymbol{\nabla} \times \mathbf{u}_{0}\right) \cdot \mathbf{k} \quad,\left.\quad \psi_{0}\right|_{S}=a(S, t)
$$

Where $\mathbf{u}_{0}$ and $a$ as before satisfy the solenoidal and the compatibility condition and $\mathbf{k}$ is the unit vector in the $\mathrm{Z}$ direction. Since the equation is fourth order elliptic, the specification of both dirichlet and Neumann conditions no longer make it overspecified. 


\subsubsection{Coupled formulation in vorticity and Stream function}

Another method to eliminate problems associated with the overdetermined problem is to solve (2.35) as a coupled equation in vorticity and stream function even in the absence of the nonlinear term. This is achieved by a unique coupling through the boundary conditions by associating one boundary condition with the transport equation and the other with the Poisson's equation. This can be written as

$$
\begin{array}{r}
\frac{\partial^{2} \boldsymbol{\nabla}^{2} \psi}{\partial t}-\nu \boldsymbol{\nabla}^{4} \psi+J\left(\boldsymbol{\nabla}^{2} \psi, \psi\right)=0 \quad \text { such that }\left.\quad \psi\right|_{S}=a \\
-\boldsymbol{\nabla}^{2} \psi=\omega \quad \text { such that }\left.\quad \frac{\partial \psi}{\partial n}\right|_{S}=b
\end{array}
$$

The spatial discretization for both these methods can be done by any of the three methods, namely, finite elements, finite differences, or spectral methods. Inspite of no direct implementation of the integral constraint, the couple formulation still satisfies the nonlocal character of vorticity. Hence further fortifying the idea of an integral condition on vorticity.

\subsubsection{Uncoupled formulation using vorticity integral conditions}

To split the two terms in the vorticity stream function formulation it becomes necessary to determine supplementary conditions for vorticity to account for its lack of boundary conditions. Quartapelle and Valz-Cris (10) came up with an integral constraint on vorticity

$$
\int \omega \eta d \Omega=\oint\left(a \frac{\partial \eta}{\partial n}-b \eta\right) d S
$$


giving the following set of linearized equations

$$
\begin{aligned}
(-\Delta+\gamma) \omega=f, \quad & \int \omega \eta d \Omega=\oint\left(a \frac{\partial \eta}{\partial n}-b \eta\right) d S \\
& -\nabla^{2} \psi=\omega,\left.\quad \psi\right|_{S}=a
\end{aligned}
$$

Where $\eta$ is any harmonic function defined in the domain $\Omega$. This is a semi implicit discretization in time with $\omega \equiv \omega_{n+1}$ and $\psi \equiv \psi^{n+1}$ at the new time level $t^{n+1}$. One of the ways to implement the integral conditions and solve the uncoupled equations is based on utilizing the linearity of the above formulation. It consists of decomposing the vorticity transport equation using the principle of superposition. The split formulation can be represented as

$$
\omega(\mathbf{x})=\omega^{0}(\mathbf{x})+\oint \omega^{6}\left(\mathbf{x} ; \zeta^{6}\right) \lambda\left(\zeta^{6}\right) d S\left(\zeta^{\prime}\right)
$$

where, $\omega^{0}$ and, $\omega^{6}$ are the solutions to

$$
\begin{array}{r}
(-\Delta+\gamma) \omega^{0}=\left.f \quad \omega^{0}\right|_{S}=0 \\
(-\Delta+\gamma) \omega^{6}=0 \quad \omega^{6}\left(\mathbf{x} ; \zeta^{6}\right)=\delta\left(s-\zeta^{6}\right)
\end{array}
$$

for any $\zeta^{6} \in S$ and $\delta$ is the dirac delta function over the boundary The value of the boundary function can then be evaluated by imposing on $\omega$ the integral constraint (2.40) with respect to all harmonic functions on the boundary which are the solution to the following problem

$$
-\nabla^{2} \eta=0 \quad \text { such that } \quad \eta(\mathbf{x} ; \zeta)=\delta(s-\zeta) \quad \text { for any } \zeta \in S
$$

And finally come up with a linear equation of the type $\mathbf{A} \boldsymbol{\lambda}=\boldsymbol{\beta}$ 
where, the value of the matrix $\mathbf{A}$ is of the form $\int \omega\left(\mathbf{x} ; \zeta^{6}\right) \eta(x ; \zeta) d \Omega$ and can be calculated and stored in the beginning.

The stream function in the above form exists only for flow in two dimensions, so the vorticity-Stream function formulation is rather difficult to implement for a three dimensional flow. Also, the vorticity is now a vector with two tangential components on the boundary. The solenoidal property of the vorticity is no longer implied by its definition but needs to be enforced. It will be shown in the next Chapter that the divergence of the vorticity vector in three dimensions can be enforced to be equal to zero by the following two boundary conditions

$$
\begin{array}{r}
\left.\boldsymbol{\nabla} \cdot \boldsymbol{\omega}\right|_{S}=0 \\
\left.\boldsymbol{\nabla} \cdot \boldsymbol{\omega}\right|_{t=0}=\boldsymbol{\nabla} \cdot\left(\boldsymbol{\nabla} \times u_{0}\right)
\end{array}
$$

But the real problem starts with the boundary conditions for the three dimensional "equivalent” for the stream function for which different schemes involving different vector potentials have been developed, each having its own set of boundary conditions and its own set of elliptic equations to solve. But each method has to come up with a set of boundary conditions for the vector function to ensure its unique solution. Apart from having to solve such complex equations with often debatable boundary conditions, these methods are also not well suited for a variational approach which often turns out to be computationally very expensive. On account of these issues with the three dimensional approach another method has begun to garner interest. This new approach, called the hybrid methods, uses a vorticity 
velocity formulation of the Navier-Stokes equations and seems to be quite well suited to two dimensional as well as three dimensional flows. This method will form the basis of this research and shall be covered in the next Chapter. 


\section{Chapter 3}

\section{Hybrid methods}

\subsection{Introduction}

These methods are based on hybrid formulations in terms of the primitive and nonprimitive variables velocity and vorticity. They are well suited for both two and three-dimensional flows. Some of the advantages of vorticity-velocity $(\omega, v)$ formulations compared to the classical formulation with primitive variables or with the nonprimitive vorticity-stream function methods (2) are:

1. Vorticity is a relevant physical variable which has been extensively studied and its distribution is of immense importance. The velocity is perhaps the most important physical variable which completely defines the kinematical problem at hand. And 
the fact that they are related by a simple elliptic equation makes this approach all the more advantageous.

2. The velocity can be supplemented by a unique set of boundary conditions as opposed to a vast number of boundary conditions necessary for a unique solution of the stream vectors or the velocity potentials.

3. The non-inertial terms caused by an accelerating reference frame enter into the flow solution through the initial and boundary conditions, without having to do anything extra to evaluate those non-inertial terms.

4. Relative ease of implementing vorticity conditions at infinity as compared to that for pressure.

But hybrid formulation also has some disadvantages too. As already mentioned, the issue with this method is the over-determined kinematic problem and the underdetermined dynamic problem. The unsteady problem in three dimensions has six unknowns compared to the four in primitive variable methods. The general formulation can be written as

$$
\begin{array}{r}
\frac{\partial \boldsymbol{\omega}}{\partial t}+\nabla \times(\boldsymbol{\omega} \times \mathbf{u})=\nu \nabla^{2} \boldsymbol{\omega} \\
\boldsymbol{\nabla} \cdot \mathbf{u}=0 \\
\boldsymbol{\omega}=\boldsymbol{\nabla} \times \mathbf{u}
\end{array}
$$


Many methods involve a Poisson's equation obtained from the curl of Equation 3.3 and utilizing (3.2) to give

$$
\nabla^{2} \mathbf{u}=-\nabla \times \omega
$$

The major problem here is ensuring the divergence condition as well as the curl of velocity. As mentioned before, the zero divergence of velocity can be ensured throughout the domain by enforcing it on the boundary. But now the solenoidal property for the vorticity also has to be ensured, which can be done in the following way.

Taking the divergence of the transport equation gives the following diffusion

$$
\frac{\partial(\boldsymbol{\nabla} \cdot \boldsymbol{\omega})}{\partial t}=\nu \boldsymbol{\nabla}^{2}(\boldsymbol{\nabla} \cdot \boldsymbol{\omega})
$$

Imposing the boundary condition $\left.\boldsymbol{\nabla} \cdot \boldsymbol{\omega}\right|_{S}=0$ on the divergence of vorticity, along with the obvious initial condition $\left.\boldsymbol{\nabla} \cdot \boldsymbol{\omega}\right|_{t=0}=\boldsymbol{\nabla} \cdot\left(\boldsymbol{\nabla} \times \mathbf{u}_{0}\right)$, should give a unique solution to the diffusion equation for $\boldsymbol{\nabla} \cdot \boldsymbol{\omega}$, i.e. $\boldsymbol{\nabla} \cdot \boldsymbol{\omega}=0$ therefore, the solenoidal property is confirmed.

Gatski (15) has classified the solution strategies into method A and method B. Method A "utilizes" the continuity and curl Equations 3.2 and 3.3 as the kinematic equations to solve and Equation 3.1 as the dynamic transport equation. Method B comprises of solving Equations 3.1 and 3.4.

Fasel (3) was among the first to publish numerical results of this method. He used the normal component of the Poisson equation for velocity and the tangential derivative of the continuity equation along with the vorticity transport equation. The boundary condition, in addition to the usual velocity condition, was simply the above-mentioned Poisson's 
equation on the boundary. Like this, many formulations based on a derived vorticity condition were formulated. Since none of these conditions are genuine constraints, many authors have solved the governing equations without using any vorticity boundary conditions whatsoever. This has led to the use of an integral constraint on the vorticity rather than a local boundary condition.

Since most of the methods do not ensure a solenoidal vorticity field by virtue of the boundary conditions (16), many authors have resorted to using a projection method to ensure the solenoidal property of vorticity. Of course, as shown by Wu, et al. (17), the non-solenoidal vorticity can be used to solve for a solenoidal velocity field. However, to find a vorticity field that is solenoidal, the vorticity is decomposed by the Helmholtz theorem to get the Poisson equation,

$$
\nabla^{2} \phi=\nabla \cdot \omega^{0}
$$

where, $\nabla \phi$ is the solenoidal part of the computed vorticity. Once $\nabla \phi$ is solved for from the above equation, the non-solenoidal vorticity can be projected onto the solenoidal field using the relation

$$
\boldsymbol{\omega}=\boldsymbol{\omega}^{0}-\nabla \phi
$$

The next section deals with some of the formulations of the vorticity-velocity methods as presented in (10) 


\subsubsection{Equations in three dimensions}

Using the above-mentioned boundary and initial conditions for vorticity along with the definition $\boldsymbol{\nabla} \times \mathbf{u}=\boldsymbol{\omega}$, the vorticity-velocity formulation in three dimensions can be represented as

$$
\begin{array}{rr}
\frac{\partial(\boldsymbol{\nabla} \cdot \boldsymbol{\omega})}{\partial t}-\nu \boldsymbol{\nabla}^{2}(\boldsymbol{\nabla} \cdot \boldsymbol{\omega})=0 & \left.\boldsymbol{\omega}\right|_{t=0}=\boldsymbol{\nabla} \times \mathbf{u}_{0} \\
\boldsymbol{\nabla}^{2} \mathbf{u}=-\boldsymbol{\nabla} \times \boldsymbol{\omega} \quad \mathbf{u}_{S}=\boldsymbol{b} & \boldsymbol{\nabla} \cdot \mathbf{u}_{S}=0 \\
\left.\boldsymbol{n} \cdot \boldsymbol{\omega}\right|_{S}=\left.\boldsymbol{n} \cdot \boldsymbol{\nabla}\right|_{S} \times \boldsymbol{b} & \left.\boldsymbol{\nabla} \cdot \boldsymbol{\omega}\right|_{S}=0
\end{array}
$$

Given the compatibilty conditions $\oint \boldsymbol{n} \cdot \boldsymbol{b} d S=0, \boldsymbol{\nabla} \cdot \mathbf{u}_{0}=0, \boldsymbol{n} \cdot \boldsymbol{b}_{t=0}=\left.\boldsymbol{n} \cdot \mathbf{u}_{0}\right|_{S}$ The above formulation can be solved numerically by a semi-implicit discretization in time and a spectral method for spatial discretization. The lack of boundary values for vorticity can be taken care of by the influence matrix technique as introduced by Daube (18). This will be briefly introduced in the next section for two dimensional flows along with a temporal discretization scheme to linearize the formulation before resolving it in space.

An uncoupled formulation using an integral constraint can be written in the following way,

$$
\begin{array}{rr}
\frac{\partial(\boldsymbol{\nabla} \cdot \boldsymbol{\omega})}{\partial t}-\nu \boldsymbol{\nabla}^{2}(\boldsymbol{\nabla} \cdot \boldsymbol{\omega})=0 & \left.\boldsymbol{\omega}\right|_{t=0}=\boldsymbol{\nabla} \times \mathbf{u}_{0} \\
\int \boldsymbol{\nabla} \times \boldsymbol{\omega} \cdot \boldsymbol{\eta} d \Omega=\oint(\boldsymbol{n} \times \boldsymbol{b} \cdot \boldsymbol{\nabla} \times \boldsymbol{\eta}+\boldsymbol{n} \cdot \boldsymbol{b} \boldsymbol{\nabla} \cdot \boldsymbol{\eta}) d S \\
\left.\boldsymbol{n} \cdot \boldsymbol{\omega}\right|_{S}=\left.\boldsymbol{n} \cdot \boldsymbol{\nabla}\right|_{S} \times \boldsymbol{b} & \left.\boldsymbol{\nabla} \cdot \boldsymbol{\omega}\right|_{S}=0 \\
\boldsymbol{\nabla}^{2} \mathbf{u}=-\boldsymbol{\nabla} \times \boldsymbol{\omega} & \mathbf{u}_{S}=\boldsymbol{b}
\end{array}
$$


Where, $\eta$ is a harmonic vector field defined by the following problem,

$$
-\nabla^{2} \eta=0, \quad \boldsymbol{n} \times\left.\boldsymbol{\eta}\right|_{S}=0
$$

The problem here is the implementation of the the integral constraint in a variational form.

The culprits are the boundary conditions $\left.\boldsymbol{n} \cdot \boldsymbol{\omega}\right|_{S}=\boldsymbol{n} \cdot \boldsymbol{\nabla}_{S} \times \boldsymbol{b}$ and $\left.\boldsymbol{\nabla} \cdot \boldsymbol{\omega}\right|_{S}=0$ which cannot be used together in a variational formulation.

\subsubsection{Equations in two dimensions}

As shown in the vorticity-stream function formulation for two dimensions, the vorticity is now a scalar variable given by $\omega=\nabla \times \mathbf{u} \cdot \boldsymbol{k}$ giving the following set of governing equations

$$
\begin{aligned}
& \left(\frac{\partial \omega}{\partial t}-\nu \nabla^{2} \omega\right) \boldsymbol{k}+\nabla \times(\omega \boldsymbol{k} \times \mathbf{u})=0, \quad \omega_{t=0}=\nabla \times \mathbf{u}_{0} \cdot \boldsymbol{k} \\
& \int \omega d \Omega=\oint \boldsymbol{\tau} \cdot \boldsymbol{b} d S \\
& -\nabla^{2} \mathbf{u}=\nabla \omega \times\left.\boldsymbol{k} \quad \boldsymbol{\nabla} \cdot \mathbf{u}\right|_{S}=\left.0 \quad \mathbf{u}\right|_{S}=\boldsymbol{b}
\end{aligned}
$$

Provided the compatibility conditions $\oint \boldsymbol{n} \cdot \boldsymbol{b} d S=0, \boldsymbol{\nabla} \cdot \mathbf{u}_{0}=0, \boldsymbol{n} \cdot \boldsymbol{b}_{t=0}=\left.\boldsymbol{n} \cdot \mathbf{u}_{0}\right|_{S}$ are met. Here $\tau$ is a unit vector tangential to the boundary.

As before, a semi-implicit dicretization can be performed by first discretizing the advection term explicitly, followed by an implicit scheme like the Crank-Nicolson scheme for the diffusion terms. 
This gives the following linearized time dicretized formulation,

$$
\begin{array}{rr}
\left(-\nabla^{2}+\gamma\right) \omega^{n+1}=f, & \text { in } \Omega \\
-\nabla^{2} \mathbf{u}^{n+1}=\nabla \omega \times \boldsymbol{k}^{n+1} & \left.\mathbf{u}\right|_{S}=\boldsymbol{b} \\
\nabla \times \mathbf{u}^{n+1}=\omega \boldsymbol{k}^{n+1} \\
\oint_{S} \boldsymbol{b}^{n+1} \cdot \boldsymbol{n} d S=0
\end{array}
$$

Just as for the primitive variable formulation, an influence matrix technique devised by Kleiser and Schumann can be used for the vorticity-velocity formulation as well. Details of its implementaion are briefly covered in Appendix B.

Solving these methods numerically, as with the three dimensional case, has not been free of problems for both the finite element and the finite difference methods. The integral constraint does offer a better representation of vorticity diffusion and its interaction with solid boundaries, there is an inclination towards using such constraints. Several innovative techniques like the staggered discretization of vorticity by Napolitano and Pascazio (10) have resulted in avoiding certain problems related to a doubly singular influence matrix in the above linear equation. The following sections describes how these problems can be solved by a new method belonging to the hybrid family. 


\subsection{The Kinematic Laplacian equation method}

The Kinematic Laplacian equation method was first introduced by Ponta in a paper (19). It is a vorticity-velocity method which decouples the evolution of vorticity from the spatial solution of a velocity field. Vorticity is advanced in time by integrating a vorticity transport equation for which an initial velocity field is obtained from the solution of the weak form of a PDE called the Kinematic Laplacian equation (the KLE). The KLE in turn is solved using the vorticity field obtained by the time integration of vorticity from the previous time step. The no-slip, no-normal flow Boundary conditions for velocity required for solving the KLE are solved over a sequence of two steps. This basically involves two integral projection in each time step ensuring compatibility of the two fields at each step. This is explained in greater detail in the following sections which are taken from a paper on KLE by F.L. Ponta (20).

\subsubsection{The constant-curl Laplacian equation}

As stated in (21), the idea behind using a Laplacian was to come up with a simple linear PDE along the lines of a potential flow equation, but, which could also account for rotational effects as seen in turbines. This lead to a Kinematic equation for solving time dependant flows over slender bodies with no flow separation under the assumption of incompressible flow and a constant curl.The following vector relation can be used to get the 
Laplacian of the velocity field

$$
\nabla^{2} \mathbf{u}=\nabla \cdot \nabla \mathbf{u}=\nabla(\nabla \cdot \mathbf{u})-\nabla \times(\boldsymbol{\nabla} \times \mathbf{u})
$$

The first and second terms can be ignored on account of the incompressibility and constant curl condition. Thus, the Laplacian $\nabla^{2} \mathbf{u}=0$ can be solved numerically under the incompressibility condition and the constant curl constraint. i.e. $\boldsymbol{\nabla} \cdot \mathbf{u}=0$ and $\boldsymbol{\nabla} \times \mathbf{u}=c$, where c is a constant.

This earlier version of KLE called the constant curl Laplacian equation (CCLE) (21), had a narrow field of application owing to the constraint of no flow separation. Nevertheless, CCLE was quite successfully used in the study of wind turbine blades (20).

\subsubsection{A generalized Laplacian $(\omega, \mathrm{u})$ method: The KLE}

As mentioned previously the KLE can be solved to get the spatial distribution of vorticity and velocity. It's a more general PDE expression than the CCLE, not limited to nonseparated flows. Consider a vorticity velocity formulation for a three dimensional NavierStokes equation for incompressible viscous flow. Consider a domain with a solid boundary $S$ and a far field external boundary $\Omega$. Therefore in a non-inertial reference frame,

$$
\frac{\partial \boldsymbol{\omega}}{\partial t}=-\mathbf{u} \cdot \nabla \boldsymbol{\omega}+\nu \nabla^{2} \boldsymbol{\omega}+\boldsymbol{\omega} \cdot \nabla \mathbf{u}
$$


If the velocity field is known at a particular time step then the above equation can be written as

$$
\frac{\partial \boldsymbol{\omega}}{\partial t}=-\mathbf{u} \cdot \boldsymbol{\nabla}(\boldsymbol{\nabla} \times \mathbf{u})+\nu \nabla^{2}(\boldsymbol{\nabla} \times \mathbf{u})+(\boldsymbol{\nabla} \times \mathbf{u}) \cdot \nabla \mathbf{u}
$$

This can be integrated in time to solve for $\omega$ at each node using an ODE solver using the vorticity and velocity field from the previous time step. However, the vorticity field so calculated is not compatible with the instantaneous boundary conditions, and so to solve for the correct vorticity and velocity fields in the spatial domain, the following Laplacian equation is used

$$
\begin{array}{r}
\nabla^{2} \mathbf{u}=\nabla \mathcal{D}-\nabla \times \omega \\
\nabla \cdot \mathbf{u}=\mathcal{D} \\
\nabla \times \mathbf{u}=\boldsymbol{\omega}
\end{array}
$$

As explained in (19) the KLE is basically a solution of the weak form of (3.17) under the simultaneous imposition of the expansion rate and the curl of the velocity i.e. the vorticity field. These constraints are given by (3.18) and (3.19).

Sections 2.4 to 2.7 of (22) gives a good explanation of the physical significance of the two constraints. Most hybrid methods simultaneously solve equations (3.17) and (3.16) under the $\boldsymbol{\nabla} \cdot \mathbf{u}=0$ constraint i.e. incompressibility. The KLE, however, as mentioned earlier solves (3.17) independent of the vorticity transport equation. Therefore the vorticity distribution given by (3.19) can be used as a second constraint along with the rate of expansion 
given by (3.18) to solve for the velocity field in space. For a brief validation, consider the decomposition of the velocity field into three orthogonal components: the irrotational component $\mathbf{u}_{\mathcal{D}}$ with zero divergence, the solenoidal component $\mathbf{u}_{\omega}$ with no vorticity and the harmonic component $\mathbf{u}_{h}$. Given the no-normal flow at the boundary along with the vorticity distribution the above mentioned decomposition i.e. $\mathbf{u}=\mathbf{u}_{\mathcal{D}}+\mathbf{u}_{\omega}+\mathbf{u}_{h}$ has a unique solution (22). (3.18) and (3.19) can be used to solve for $\mathbf{u}_{\mathcal{D}}$ and $\mathbf{u}_{\omega}$ as

$$
\begin{aligned}
\nabla \cdot \mathbf{u} & =\nabla \cdot \mathbf{u}_{\mathcal{D}}=\mathcal{D} \\
\nabla \times \mathbf{u} & =\nabla \times \mathbf{u}_{\omega}=\boldsymbol{\omega} .
\end{aligned}
$$

For $\mathbf{u}_{h}$ substitute the above mentioned decomposition in to (3.16),

$$
\begin{aligned}
\nabla^{2}\left(\mathbf{u}_{h}+\mathbf{u}_{\mathcal{D}}+\mathbf{u}_{\omega}\right) & =\nabla^{2} \mathbf{u}_{h}+\nabla\left(\nabla \cdot \mathbf{u}_{\mathcal{D}}\right)-\nabla \times\left(\nabla \times \mathbf{u}_{\omega}\right) \\
& =\nabla \mathcal{D}-\nabla \times \omega
\end{aligned}
$$

Substituting (3.20) and (3.21) in (3.22) gives,

$$
\nabla^{2} \mathbf{u}_{h}=0
$$

This Laplacian equation gives the solution for $\mathbf{u}_{h}$. Therefore the KLE ensures a complete and unique solution of the velocity field.

To impose the no-normal flow and no-slip velocity boundary conditions on $S$ together with the correspondingly compatible boundary conditions on the vorticity, a solution method based on two consecutive solutions of the KLE is used: the first under free-slip and the second under no-slip boundary conditions on the solid surface. The algorithmic sequence explained below $(19,20)$ is iteratively performed at each time step within an iterative time 
integration performed by an adaptive variable-stepsize ODE solver for incompressible flow.

1. The vorticity is advanced in time by integrating (3.16) in time at each node in space to get an initial vorticity field $\tilde{\boldsymbol{\omega}}^{n}$ field. Since velocity from the $(n-1)^{\text {st }}$ time step is used to get vorticity for step $n$, this field is not compatible with the velocity boundary conditions.

2. Enforce homogeneous conditions $\tilde{\boldsymbol{\omega}}^{n}$ at the boundary surface to get $\tilde{\boldsymbol{\omega}}_{0}^{n}$. This is done by imposing a zero boundary value for vorticity at each node on the boundary.

3. Applying the no normal flow velocity boundary conditions and setting $\frac{\partial u_{x}}{\partial \boldsymbol{n}}=0$ on the solid boundary, equation (3.17) i.e. the KLE is solved for $\overrightarrow{\boldsymbol{u}}^{n}$ under the 2 constraints given by (3.18) and (3.19) using $\tilde{\boldsymbol{\omega}}_{0}^{n}$ as the vorticity field. Here $u_{x}$ is the tangential component of $\boldsymbol{u}$.

4. Using this $\overrightarrow{\boldsymbol{u}}^{n}$ the vorticity field is again calculated as $\boldsymbol{\omega}^{n}=\boldsymbol{\nabla} \times \overrightarrow{\boldsymbol{u}}^{n}$, only this time both boundary conditions i.e. no-normal flow $\boldsymbol{u} . \boldsymbol{n}=0$ and the no slip condition $\boldsymbol{u} . \boldsymbol{\tau}=0$ are applied on $S$. This $\boldsymbol{\omega}^{n}$ can be seen as a vorticity field produced as an effect of the slip induced in the previous step, somewhat like the vorticity creation methods $(23,24,25)$.

5. Using the above calculated vorticity field $\boldsymbol{\omega}^{n}$ a fixed velocity field $\boldsymbol{u}^{n}$ is computed as a solution to the KLE using both constraints and the two boundary conditions i.e. the no normal flow and the no slip condition. 
For the velocity boundary condition on the far field external boundary $S_{\infty}$, the corresponding Dirichlet conditions are applied.

The above algorithm clearly shows the vorticity in time and velocity in space approach of KLE. The momentum equation is solved in step 1 itself. Step 2-5 consist of solving the KLE for each time step to get the spatial distribution of velocity, compatible with both the vorticity distribution as well as the velocity boundary conditions. Setting vorticity equal to zero at boundary is consistent with the free slip boundary condition for velocity in step 2 and finally as in vorticity creation methods, the no slip condition of step 4 gives the final vorticity field in response to the slip induced in step 3. It can be seen that the two solutions of KLE , each with a different set of boundary conditions take care of the vorticity boundary conditions also. These two integral projections on the velocity field ensure a vorticity compatible with the velocity boundary conditions in each time step. This decoupling between the vorticity evolution and the solution to get velocity distribution along with the compatible vorticity distribution, makes it possible to solve problems with different constitutive relations using this method since the physics involved in any such relation is independent of the spatial solution of KLE. It also becomes much simpler to implement the variational formulation since the PDE system now does not depend either on time or the constitutive relations, but are simply a set of kinematic equations. Since this method is integral and not limited to just the boundary data for calculating boundary vorticity, it does seem to have a somewhat better physical interpretation then the other vorticity generation methods (10). 


\subsubsection{Variational formulation of KLE}

A variational form of (3.17) can be written using the Galerkin method (20) as follows,

$$
\int_{\Omega}(\boldsymbol{\nabla} \cdot \boldsymbol{\nabla u}) \cdot \boldsymbol{\delta} \boldsymbol{u} \mathrm{d} \Omega=-\int_{\Omega}(\boldsymbol{\nabla} \times \boldsymbol{\omega}) \cdot \boldsymbol{\delta} \boldsymbol{u} \mathrm{d} \Omega
$$

where $\boldsymbol{\delta} \boldsymbol{u}$ is a virtual, arbitrary velocity field on $\Omega$ that is set to zero where ever Dirichlet conditions are applied. The next step would be to integrate the left hand side of (3.24) by parts and using the divergence theorem to get, $\boldsymbol{\delta} \boldsymbol{u}$ vanishes on $S_{\infty}$,

$$
\int_{\Omega} \nabla \mathbf{u}: \nabla \boldsymbol{\delta} \boldsymbol{u} \mathrm{d} \Omega-\int_{S} \boldsymbol{n} \cdot \boldsymbol{\nabla} \mathbf{u} \cdot \boldsymbol{\delta} \boldsymbol{u} \mathrm{d} S=\int_{\Omega}(\boldsymbol{\nabla} \times \boldsymbol{\omega}) \cdot \boldsymbol{\delta} \boldsymbol{u} \mathrm{d} \Omega .
$$

The no slip (as well as the free slip) and no normal flow boundary conditions ensure that $\boldsymbol{\delta} \boldsymbol{u}=0$, thereby reducing (3.25) to:

$$
\int_{\Omega} \nabla \mathbf{u}: \nabla \boldsymbol{\delta} \boldsymbol{u} \mathrm{d} \Omega=\int_{\Omega}(\boldsymbol{\nabla} \times \boldsymbol{\omega}) \cdot \boldsymbol{\delta} \boldsymbol{u} \mathrm{d} \Omega
$$

The Laplacian operator also has an equivalent minimization formulation which gives for the variational form of KLE the following functional,

$$
\Pi=\int_{\Omega} \frac{1}{2} \nabla \mathbf{u}: \nabla \mathbf{u} \mathrm{d} \Omega-\int_{\Omega}(\boldsymbol{\nabla} \times \boldsymbol{\omega}) \cdot \mathbf{u} \mathrm{d} \Omega .
$$

To impose the constraints (3.18) and (3.19) the penalty method was used over other possible schemes. A brief on why it is preferred over other more rigorous alternatives can be found in (2). The penalty terms according to the two constraints given by (3.18) and(3.19) are added to (3.27) giving the modified functional $\tilde{\Pi}$ as,

$$
\tilde{\Pi}=\Pi+\int_{\Omega} \frac{\alpha_{\mathcal{D}}}{2}(\boldsymbol{\nabla} \cdot \mathbf{u})^{2}+\frac{\alpha_{\omega}}{2}(\boldsymbol{\nabla} \times \mathbf{u}-\boldsymbol{\omega}) \cdot(\boldsymbol{\nabla} \times \mathbf{u}-\boldsymbol{\omega}) \mathrm{d} \Omega
$$


Where the penalty constants are given by $\alpha_{\omega}$ and $\alpha_{\mathcal{D}}$ The stationary of $\tilde{\Pi}$ with respect to $\mathbf{u}$ can be written as,

$$
\begin{gathered}
\delta \tilde{\Pi}=\int_{\Omega} \boldsymbol{\nabla u}: \boldsymbol{\nabla} \boldsymbol{\delta} \boldsymbol{u}-(\boldsymbol{\nabla} \times \boldsymbol{\omega}) \cdot \boldsymbol{\delta} \boldsymbol{u}+\alpha_{\mathcal{D}}(\boldsymbol{\nabla} \cdot \mathbf{u})(\boldsymbol{\nabla} \cdot \boldsymbol{\delta} \boldsymbol{u}) \\
\quad+\alpha_{\omega}(\boldsymbol{\nabla} \times \mathbf{u}-\boldsymbol{\omega}) \cdot(\boldsymbol{\nabla} \times \boldsymbol{\delta} \boldsymbol{u}) \mathrm{d} \Omega=0 .
\end{gathered}
$$

Reordering the above equation gives,

$$
\begin{aligned}
& \int_{\Omega} \boldsymbol{\nabla u}: \nabla \boldsymbol{\delta} \boldsymbol{u}+\alpha_{\mathcal{D}}(\boldsymbol{\nabla} \cdot \mathbf{u})(\boldsymbol{\nabla} \cdot \boldsymbol{\delta} \boldsymbol{u})+\alpha_{\omega}(\boldsymbol{\nabla} \times \mathbf{u}) \cdot(\boldsymbol{\nabla} \times \boldsymbol{\delta} \boldsymbol{u}) \mathrm{d} \Omega= \\
& \int_{\Omega}(\boldsymbol{\nabla} \times \boldsymbol{\omega}) \cdot \boldsymbol{\delta} \boldsymbol{u}+\alpha_{\omega} \boldsymbol{\omega} \cdot(\boldsymbol{\nabla} \times \boldsymbol{\delta} \boldsymbol{u}) \mathrm{d} \Omega
\end{aligned}
$$

(3.30) gives the variational formulation for KLE for incompressible flow, with (3.18) and (3.19) as the constraints. As mentioned before, this variational form can be solved by a spatial discretization using finite elements or spectral methods. This thesis deals with a spectral element approach which will be introduced in the next chapter.

Even though in previous paragraphs the KLE was referred to as a "vorticity-in-time/velocityin-space split approach”, this is more a general description of its time-space/vorticityvelocity uncoupled nature than a strict definition of its algorithmic structure. Strictly speaking, time-marching splitting or fractional-step methods replace simultaneous processes by sequential steps as a means to increase efficiency (26). Split may be by dimensions (e.g. a three-dimensional process split into three one-dimensional substeps), or by physics (e.g. advection on one fractional step, pressure adjustment on another, and diffusion on a third). For the hydrodynamic equations, the advantage of splitting-by-process is that the nonlinear advection process can be treated by a different algorithm than pressure adjustment, which 
in turn can be different from diffusion, the latter two involve a linear solution each. The advective step is usually advanced explicitly and the adjustment of fields, is integrated implicitly. A typical example of this technique is the very successful AB3CN (third-order Adams-Bashforth/Crank-Nicholson) three-step scheme (see (27, 28), among others). Besides its advantages, splitting also has some drawbacks, mostly related with consistency and the treatment of boundary conditions (see (26), Sec. 13.1-13.4). The choice of appropriate boundary conditions is quite important in minimizing the splitting error, as shown by Karniadakis et al. (29), where high-order pressure boundary conditions are found to be the key to the time accuracy of the splitting scheme.

Contrarily, there is no splitting whatsoever in the KLE method. All terms in the physical problem are solved simultaneously during time integration of the vorticity field, and all the spatial components of the velocity are solved together by the KLE. Since the KLE is an entirely Kinematic equation with the entire physics concerned with any of the nonlinearities and complex constitutive relations limited to the time integration schemes, it favors modeling complex flow problems like non-Newtonian flows or turbulent flows etc. Since it is also a universal vector equation, basically any field represented by this relation can be solved for as long as the divergence and curl of that field has a solvable transport equation. Also, since the vector relation is independent of the time iteration process, the KLE can be coupled with other processes like heat transfer or chemical processes by simply adding the required relation to the existing ODE system. So basically just the source term to the KLE is changed to solve different physical problems. 


\section{Chapter 4}

\section{Numerical implementation of the KLE}

\subsection{Introduction}

This chapter deals with the numerical implementation of the KLE using a spectral element method to discretize Equation 3.30 in space along with a predictor-corrector time integration scheme. The spatial discretization scheme used here is a two dimensional isoparametric spectral element with a high order Lagrangian polynomial to interpolate solutions within the element. An isoparametric element was chosen because of the complex differential equations involved as also the complexity of the intended surfaces to be modeled. The main advantage of this type of an element is that the integration has to be performed over the "parent" element which represents a normalized domain in terms of a local co-ordinate 


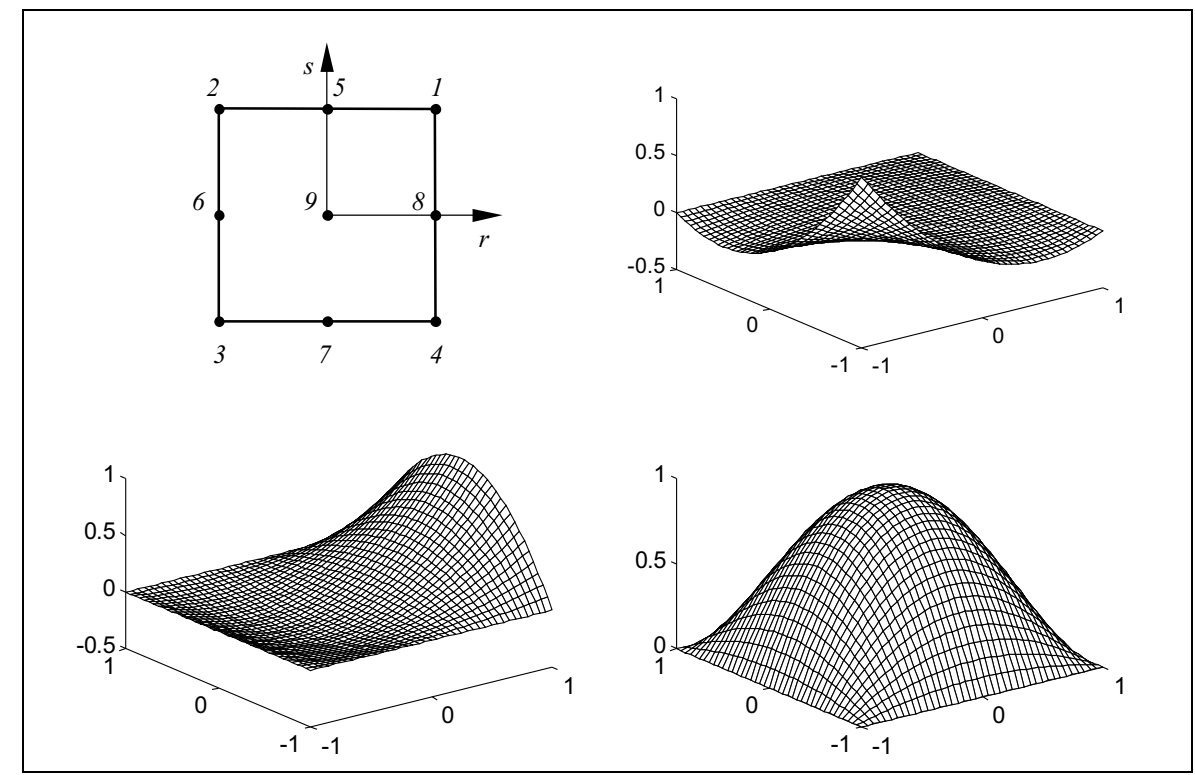

Figure 4.1: A two-dimensional nine node isoparametric element in its natural coordinate system along with a graphical representation of three of its nine interpolation functions i.e nodes 3,8 and 9.

system varying between +1 and -1 . This makes it easier to implement any numerical technique. An isoparametric element uses the same Lagarange polynomial (shape functions) to interpolate the unknown variable within the elements as the ones used to map the global to local coordinates. Figure 4.1 shows an example of biquadratic interpolation functions of a nine-node isoparametric quadrilateral element on its natural system of coordinates, i.e. $(\mathrm{r}, \mathrm{s})$. A quadrilateral element was chosen because of its high convergence rate and its ability to reduce the skin error on curvilinear bondaries when compared to linear elememts. Nevertheless, other discretization techniques may be applied to the implementation of the KLE method. For our experimentation, the spectral element method is used for the KLE which will be discussed in the following section. 


\subsection{The Spectral-element method for KLE}

The general trend in finite element methods had been to use Lagrange polynomials of a particular order as shape functions. To improve the accuracy, the number of elements was increased. This is called an $h$-type finite element method. For sufficiently smooth problems

these methods converge at an algebraic rate with the error being proportional to $\frac{1}{N^{p+1}}$ where $N$ is the number of degrees of freedom and $p$ is the order of the Lagrange polynomial (30). Another approach would be to follow the $h$-type discretization with an increase in the order of the interpolating polynomial within each element to improve accuracy. Again, for sufficiently smooth solutions this would give an exponential convergence rate (30). These are called the $p$-type methods. The spectral method is a particular implementation of the $p$-version of an $h p$ finite element method.

The spectral-element method was introduced some twenty years back (31, 32). It's main purpose was to tackle complicated domains which the spectral methods were not able to handle. As shown in (33) this $h-p$ type of method was capable of local refinements, and where thus good for complex geometries and at the same time preserved the high convergene rates seen in spectral methods. Owing to the $h-p$ discretization, a high accuracy can be achieved for less number of nodes, amking it a highly memory-minimizing method (26).

Mostly the Legendre or Chebyshev polynomials are used by the spectral element methods in order to come up with suitable basis functions. The same points are used for the 
interpolation functions as are for the numerical integration within the elements. These collocation points are called the Gauss-Legendre-Lobatto (GLL) quadrature points. This leads to diagonal mass matrices making the system more efficient.

As mentioned at the beginning of this chapter, in this particular analysis an isoparametric element is used with the Lagrangian polynomials as interpolating functions for the solution. The variational formulation for the KLE using the Galerkin method, shown in the previous chapter, is used to solve for the velocity field at the nodal points. The nodes are at the GLL points.For higher order elements using the GLL points in place of the regular equispaced points is more economical (34). Giraldo (35), through experiments has shown that for higher order interpolating polynomials (in excess of 4) the solution results for Gauss Legendre and GLL quadrature are comparable.

As shown in (20) the finite-element discretization of the velocity field and its gradient can be represented as,

$$
\mathbf{u}=\left[\begin{array}{l}
u_{x} \\
u_{y}
\end{array}\right]=\mathbf{H} \cdot \mathbf{U}^{e}, \quad \boldsymbol{\nabla} \mathbf{u}=\left[\begin{array}{c}
\frac{\partial u_{x}}{\partial x} \\
\frac{\partial u_{x}}{\partial y} \\
\frac{\partial u_{y}}{\partial x} \\
\frac{\partial u_{y}}{\partial y}
\end{array}\right]=\mathbf{B} \cdot \mathbf{U}^{e},
$$

where $\mathbf{H}$ is the interpolation-function, $\mathbf{B}$ it's derivative and $\mathbf{U}^{e}$ is the array of discretized velocity at nodes of each element, 
$\mathbf{U}^{e}=\left[\begin{array}{c}u_{x}^{1} \\ u_{y}^{1} \\ u_{x}^{2} \\ \vdots \\ u_{x}^{N G L^{2}} \\ u_{y}^{N G L^{2}}\end{array}\right]$

$$
\mathbf{H}=\left[\begin{array}{cccccc}
h^{1} & 0 & h^{2} & \cdots & h^{N G L^{2}} & 0 \\
0 & h^{1} & 0 & \cdots & 0 & h^{N G L^{2}}
\end{array}\right]
$$

$$
\mathbf{B}=\left[\begin{array}{cccccc}
\frac{\partial h^{1}}{\partial x} & 0 & \frac{\partial h^{2}}{\partial x} & \cdots & \frac{\partial h^{N G L^{2}}}{\partial x} & 0 \\
\frac{\partial h^{1}}{\partial y} & 0 & \frac{\partial h^{2}}{\partial y} & \cdots & \frac{\partial h^{N G L^{2}}}{\partial y} & 0 \\
0 & \frac{\partial h^{1}}{\partial x} & 0 & \cdots & 0 & \frac{\partial h^{N G L^{2}}}{\partial x} \\
0 & \frac{\partial h^{1}}{\partial y} & 0 & \cdots & 0 & \frac{\partial h^{N G L^{2}}}{\partial y}
\end{array}\right]
$$

where $N G L=p+1$ is the number of nodes of the Gauss-Lobatto interpolation.

The elements of (4.3) are given by,

$$
\left[\begin{array}{c}
\frac{\partial h^{k}}{\partial x} \\
\frac{\partial h^{k}}{\partial y}
\end{array}\right]=\mathbf{J}^{-1} \cdot\left[\begin{array}{c}
\frac{\partial h^{k}}{\partial r} \\
\frac{\partial h^{k}}{\partial s}
\end{array}\right], \quad k=1, \ldots, N G L^{2},
$$

where $\mathbf{J}$ is the Jacobian operator which relates the natural to the local coordinate derivates,

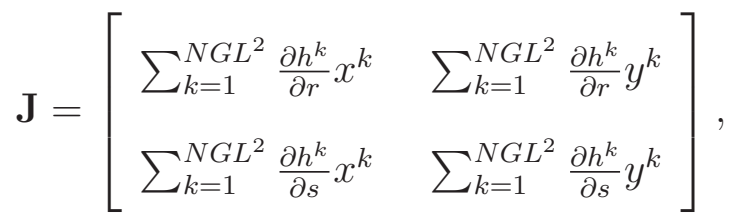


and $\left(x^{k}, y^{k}\right)$ the local coordinates of the nodes. The divergence of the velocity field is given by

$$
\boldsymbol{\nabla} \cdot \mathbf{u}=\mathbf{m} \cdot \mathbf{B} \cdot \mathbf{U}^{e}, \quad \mathbf{m}=\left[\begin{array}{llll}
1 & 0 & 0 & 1
\end{array}\right]
$$

and the curl of the velocity $\omega_{z}$ (the only component of the curl not equal to zero), is obtained as,

$$
\boldsymbol{\nabla} \times \mathbf{u}=\mathbf{r} \cdot \mathbf{B} \cdot \mathbf{U}^{e}, \quad \mathbf{r}=\left[\begin{array}{llll}
0 & -1 & 1 & 0
\end{array}\right]
$$

Similarly for vorticity,

$$
\boldsymbol{\omega}=\mathbf{H}_{\omega} \cdot \boldsymbol{\omega}^{e}, \quad \boldsymbol{\nabla} \times \boldsymbol{\omega}=\left[\begin{array}{c}
\frac{\partial \omega}{\partial y} \\
-\frac{\partial \omega}{\partial x}
\end{array}\right]=\mathbf{B}_{\omega} \cdot \boldsymbol{\omega}^{e},
$$

where $\omega^{e}$ gives the vorticity values at nodes of each element calculated by integrating in time the vorticity transport equation, and as shown for velocity $\mathbf{H}_{\omega}$ and $\mathbf{B}_{\omega}$ are the vorticity interpolation-functions snd their derivatives respectively,

$$
\begin{gathered}
\boldsymbol{\omega}^{e}=\left[\begin{array}{c}
\omega^{1} \\
\omega^{2} \\
\vdots \\
\omega^{N G L^{2}}
\end{array}\right], \quad \mathbf{H}_{\omega}=\left[\begin{array}{llll}
h^{1} & h^{2} & \ldots & h^{N G L^{2}}
\end{array}\right], \\
\mathbf{B}_{\omega}=\left[\begin{array}{cccc}
\frac{\partial h^{1}}{\partial y} & \frac{\partial h^{2}}{\partial y} & \ldots & \frac{\partial h^{N G L^{2}}}{\partial y} \\
-\frac{\partial h^{1}}{\partial x} & -\frac{\partial h^{2}}{\partial x} & \ldots & -\frac{\partial h^{N G L^{2}}}{\partial x}
\end{array}\right] .
\end{gathered}
$$

For the finite element analysis, first each element can be thought of as a discretized sub- 
domain $\left(\Omega^{e}\right)$. Therefore if equation (3.30) is considered at each $\Omega^{e}$ and the corresponding discretized values of the velocity and vorticity fields are substituted for, the following equation is obtained,

$$
\boldsymbol{\delta} \mathbf{U}^{e T} \cdot \underbrace{\left(\mathbf{K}_{L}^{e}+\mathbf{K}_{\mathcal{D}}^{e}+\mathbf{K}_{\omega}^{e}\right)}_{\mathbf{K}^{e}} \cdot \mathbf{U}^{e}=\delta \mathbf{U}^{e T} \cdot \underbrace{\left(\mathbf{R}_{L}^{e}+\mathbf{R}_{\omega}^{e}\right)}_{\mathbf{R}^{e}} \cdot \boldsymbol{\omega}^{e},
$$

where

$$
\begin{aligned}
\mathbf{K}_{L}^{e} & =\int_{\Omega^{e}} \mathbf{B}^{T} \cdot \mathbf{B} \mathrm{d} \Omega=\int_{-1}^{1} \int_{-1}^{1} \mathbf{B}^{T} \cdot \mathbf{B}|\mathbf{J}| \mathrm{d} r \mathrm{~d} s \\
\mathbf{K}_{\mathcal{D}}^{e} & =\int_{-1}^{1} \int_{-1}^{1} \alpha_{\mathcal{D}} \mathbf{B}^{T} \cdot \mathbf{m}^{T} \cdot \mathbf{m} \cdot \mathbf{B}|\mathbf{J}| \mathrm{d} r \mathrm{~d} s \\
\mathbf{K}_{\omega}^{e} & =\int_{-1}^{1} \int_{-1}^{1} \alpha_{\omega} \mathbf{B}^{T} \cdot \mathbf{r}^{T} \cdot \mathbf{r} \cdot \mathbf{B}|\mathbf{J}| \mathrm{d} r \mathrm{~d} s \\
\mathbf{R}_{L}^{e} & =\int_{-1}^{1} \int_{-1}^{1} \mathbf{H}^{T} \cdot \mathbf{B}_{\omega}|\mathbf{J}| \mathrm{d} r \mathrm{~d} s \\
\mathbf{R}_{\omega}^{e} & =\int_{-1}^{1} \int_{-1}^{1} \alpha_{\omega} \mathbf{B}^{T} \cdot \mathbf{r}^{T} \cdot \mathbf{H}_{\omega}|\mathbf{J}| \mathrm{d} r \mathrm{~d} s
\end{aligned}
$$

$\boldsymbol{\delta} \boldsymbol{U}^{e}$ gives the array of values at nodes of each element for the arbitrary $\boldsymbol{\delta} \boldsymbol{u}$.

The arrays and the matrices of (4.11) are assembled for each element to give the following global system,

$$
\mathbf{K} \cdot \mathbf{U}^{e}=\mathbf{R} \cdot \boldsymbol{\omega}
$$

As mentioned earlier a quadrilateral element has high convergence rate and reduces the skin error on circular boundaries. At the same time triangular elements find it easier to change mesh density in a more smooth and gradual manner and are also more suitable for unstructured meshing $(20,21)$. Thus the domain was first dicretized using triangular elements which was subsequently converted to a quadrilateral mesh, by dividing each tri- 

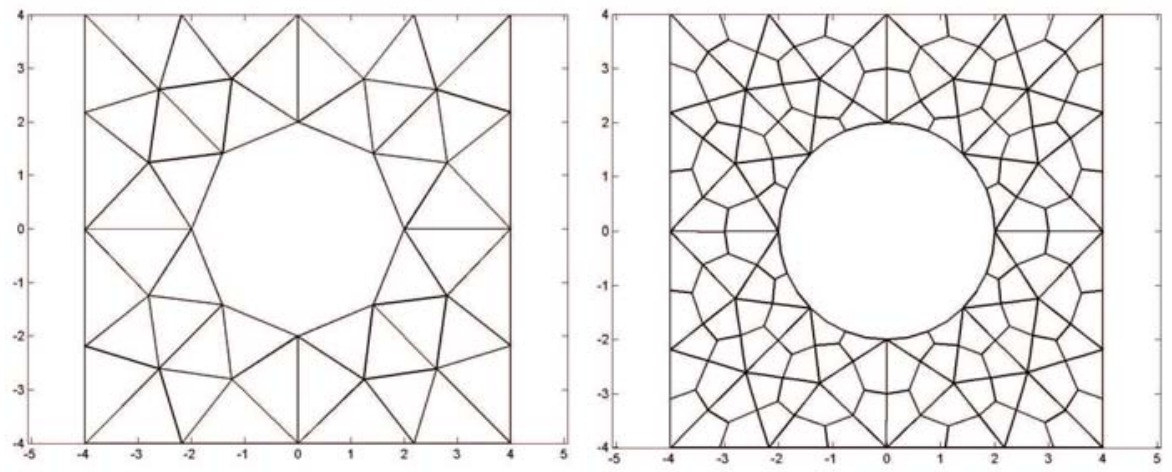

Figure 4.2: A tri-quadrilateral finite element mesh derived from an unstructured triangular mesh.

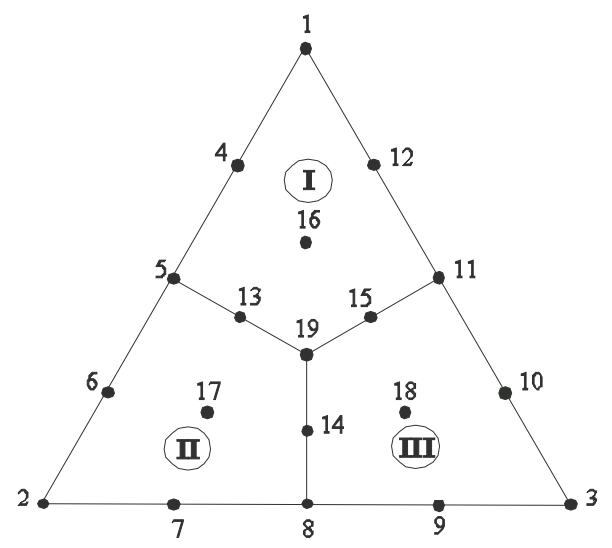

Figure 4.3: The internal topology of a tri-quadrilateral element. Quadrilateral elements (I)-(III) are the nine-node isoparametric elements. 1-19 is the in-triangle global numbering of the nodes. angle into three quadrilaterals. This can be seen in figure (4.2). An important advantage of this "tri-quadrilateralization" is a process called static condensation of internal nodes. These nodes lying inside the triangle though used for elemental integration are not used while assembling the final global structure matrices. These are later recovered from the values obtained by the solution of the external nodes. To establish an equation for this 
condensation, as shown in (36), the system $\mathbf{K}^{e} \cdot \mathbf{U}^{e}=\mathbf{R}^{e} \cdot \boldsymbol{\omega}^{e}$ are partitioned as,

$$
\left[\begin{array}{ll}
\mathbf{K}_{a a}^{e} & \mathbf{K}_{a b}^{e} \\
\mathbf{K}_{b a}^{e} & \mathbf{K}_{b b}^{e}
\end{array}\right] \cdot\left[\begin{array}{l}
\mathbf{U}_{a}^{e} \\
\mathbf{U}_{b}^{e}
\end{array}\right]=\left[\begin{array}{l}
\mathbf{R}_{a}^{e} \\
\mathbf{R}_{b}^{e}
\end{array}\right] \cdot \boldsymbol{\omega}^{e}
$$

where $a$ is for the degrees of freedom 1-24 of the velocity at nodes 1-12 and $b$ is for the degrees of freedom 25-38 of the velocity at nodes 13-19. The second row of the above equation gives,

$$
\mathbf{U}_{b}^{e}=\underbrace{\left(\mathbf{K}_{b b}^{e}\right)^{-1} \cdot \mathbf{R}_{b}^{e}}_{\mathbf{R}_{b}^{e}} \cdot \boldsymbol{\omega}^{e}-\underbrace{\left(\mathbf{K}_{b b}^{e}\right)^{-1} \cdot \mathbf{K}_{b a}^{e}}_{\mathbf{K}_{b a}^{e}} \cdot \mathbf{U}_{a}^{e},
$$

substituting this into the first row of (4.13) and reordering,

$$
\begin{aligned}
& \underbrace{\left(\mathbf{K}_{a a}^{e}-\mathbf{K}_{a b}^{e} \cdot\left(\mathbf{K}_{b b}^{e}\right)^{-1} \cdot \mathbf{K}_{b a}^{e}\right)}_{\mathbf{K}^{e}} \cdot \mathbf{U}_{a}^{e}= \\
& \underbrace{\left(\mathbf{R}_{a}^{e}-\mathbf{K}_{a b}^{e} \cdot\left(\mathbf{K}_{b b}^{e}\right)^{-1} \cdot \mathbf{R}_{b}^{e}\right)}_{\mathbf{R}^{e}} \cdot \boldsymbol{\omega}^{e},
\end{aligned}
$$

This is the condensed form. Assembling the arrays and matrices of (4.14) and (4.15) gives the following global condensed system,

$$
\begin{gathered}
\mathbf{K} \cdot \mathbf{U}_{a}=\mathbf{R} \cdot \boldsymbol{\omega}, \\
\mathbf{U}_{b}=\mathbf{R}_{b} \cdot \boldsymbol{\omega}-\mathbf{K}_{b a} \cdot \mathbf{U}_{a},
\end{gathered}
$$

The static condensation process leads to almost a $40 \%$ reduction in the size of the global system to be solved and also leads to a better condition number of the global structure matrices. This is in accordance with the Schur complement method, where the condensed matrix forms the Schur complement for the in-triangle nodes of the original system. As 
mentioned earlier none of the structure matrices depend on the physics of the problem, in this case the vorticity or the time, they can be calculated and stored and used over and

over again as required. Since $\mathrm{KK}$ is positive definite and symmetric it is factorized by the Cholesky decomposition method and the factor (triangular) so obtained can be used to solve for $\mathbf{U}_{a}$. One problem with the spectral element methods is the loss of the exponential convergence and also the higher accuracy in case of singularities like shock in compressible flow (37). This is often seen while interpolating non-smooth functions (abrupt changes in boundaries and forces etc.) using high order polynomials.

\section{Evaluating the right-hand side of the vorticity transport equation}

As shown in (20) for the two-dimensional implementation of the time-integration procedure, the vorticity transport Equation (3.16) can be written in a more convenient way as follows,

$$
\frac{\partial \boldsymbol{\omega}}{\partial t}=\boldsymbol{F}(\boldsymbol{\omega}, t)=\boldsymbol{\nabla} \times(\nu \nabla \cdot \nabla \mathbf{u}-\mathbf{u} \cdot \nabla \mathbf{u})
$$

The RHS of (4.18)is solved for by carrying out the respective curl, divergence and gradient operations on the discretized counterpart of $u$ as found by the KLE algorithm previously explained. Since, for the spectral-element case, the Gauss-Lobatto points are the same as the nodes,therefore, for those lying on the inter-element boundaries, an average of the values from elements sharing those boundaries can be used.

The weight of each Gaussian point depends on the mesh geometry and is calculated during 
assembly. So the arrays for the differential operators are assembled at the same time as the Finite element matrices. Those arrays perform the differential operations on any vector or tensor field, as a dot product with the corresponding discrete solution of that field. For instance, the discrete form of the curl of the velocity field $\boldsymbol{\nabla} \times \mathbf{u}$ is given by the dot product $C_{u r l} \cdot$ U. Thus, the discrete form of (4.18) is written as,

$$
\mathbf{F}(\boldsymbol{\omega}, t)=\mathbf{C}_{u r l} \cdot\left(\nu \mathbf{D}_{i v}-\mathbf{U}_{a d v}\right) \cdot \mathbf{G}_{r a d} \cdot \mathbf{U},
$$

where $\mathbf{G}_{r a d}$ gives the gradient, $\mathbf{D}_{i v}$ the divergence of $\mathbf{G}_{r a d}, \mathbf{C}_{u r l}$ the curl vector and $\mathbf{U}_{a d v}$ is obtained by reordering $\mathbf{U}$ to perfrom the dot product $\mathbf{u} \cdot \nabla \mathbf{u}$ in the advective term.

Since none of them depend on the vorticity field or time, they can be, as with the structure matrices, calculated and stored for further use. For the time integration Adams-BashforthMoulton predictor-corrector (ABM-PECE) solver with multivariable order and adaptive stepsize is used and the results show that it is efficient enough to pursue further research (2) as discussed in the following sections. 


\section{Chapter 5}

\section{Experiment and analysis}

\subsection{Introduction}

This chapter deals with a numerical experiment performed with the aim of determining a quality metric along with its threshold value, to be used in the mesh generation algorithm for solving the KLE. Since this algorithm does not really incorporate the solution field of the given problem, the experiment aims at testing certain metrics by seeing how well the quality value of the elements, obtained using different measures, matches with the solution of the KLE problem.

While difficult to give an absolute definition for mesh quality, it is safe to say that a good quality mesh should accurately and efficiently solve the given differential equation, thereby 
suitably simulating the concerned physical problem. The following important aspects regarding mesh quality are mentioned by Knupp (38):

1. The mesh quality depends on the problem at hand.

2. The mesh should not create difficulties for the simulation. Such as, inverted elements, high condition numbers, or clusters of large eigenvalues.

3. The mesh should result in sufficiently accurate solutions.

The first point is the reason behind performing the controlled distortion tests. Since the quality metric should be problem specific, a series of runs of the KLE are performed on a 'distorted' mesh. Since the KLE uses unstructured meshes, the main aim of the experiment is to suitably quantify the distortion in order to prevent a mesh with poor quality elements that have inverted edges, angles excessively large or small, poor aspect ratios, etc. The experiment mainly comprises of performing a controlled distortion on a regular mesh, solving the KLE for this mesh, and comparing the results with the quality obtained for that mesh using various metrics. The metric that best reflects the results obtained is then used to measure the quality of elements in the unstructured mesh.

As mentioned in Knupp‘s paper (38) two important functions expected of a quality metric are:

1. Identifying defects like ill-conditioned elements, inverted edges, extreme values of 
angles and bad topology.

2. As a guiding factor to implement various smoothing and optimization techniques.

While solving the KLE in an unstructured mesh, several factors affect the result. The three important ones guiding the design of this experiment are the h-refinement, the p-refinement, and the amount of distortion. As mentioned in the previous chapter, this thesis is concerned with the spectral element implementation of the KLE, therefore both the $h$ and the p refinement methods are equally important. Since the quality metric should reflect the effect of distortion on the solution, one must consider the effect an h-p refinement has on factors like error, convergence rates, solution times, etc. It is important to bear in mind that while solving real problems in a distorted mesh, one might have to tinker with the number of elements and the order of interpolation, thereby making all three factors equally important. Keeping these things in mind, the controlled distortion experiment was performed and a suitable quality metric, conforming to the solution of the KLE, was decided upon. This experiment, along with the effect of distortion on the net error norm of the estimated velocity and the condition number of the mesh is dealt with in the subsequent sections. 


\subsection{Controlled distortion experiments}

The idea behind a controlled distortion is to distort, in a step by step fashion, a regular structured mesh. The KLE is used to solve a canonical flat plate problem for each distortion. As mentioned earlier the reason for distorting the mesh in a controlled manner is not just to come up with a suitable quality measure but also decide upon a suitable threshold value of the distortion to help improve the element quality. For this purpose several different types of distortions were considered,and finally the one shown in Figure 5.1 was considered as the test distortion.

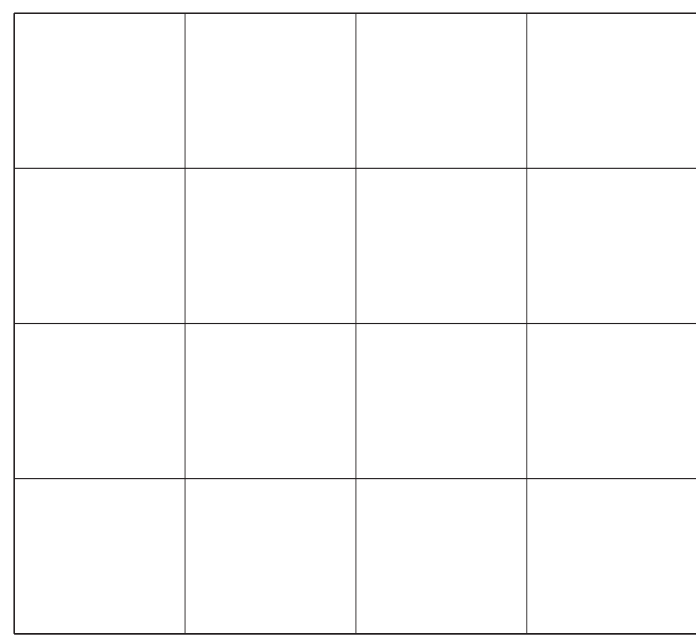

(a) A structured mesh with zero distortion

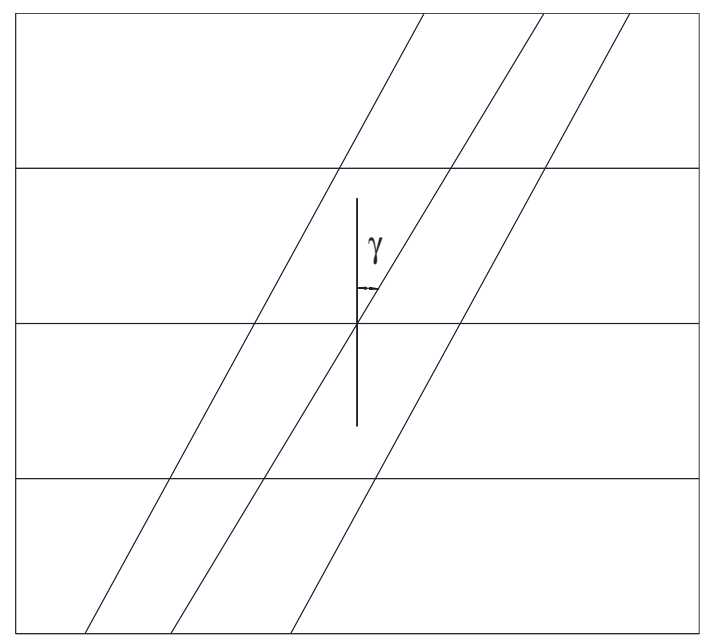

(b) Distorted Mesh

Figure 5.1: A Structured mesh being distorted by $\gamma$ radians

This distortion is specifically designed to ensure that the elements in the mid-region are the most skewed elements and, as we move towards the edges the aspect ratio starts to dominate as the element distorting factor. This makes it easier to compare various quality parameters 
used on a given mesh, especially if one is interested in ensuring that the metric should represent skewness rather than aspect ratio as the main cause of bad quality elements. The most useful feature of the distortion shown in Figure 5.1 is that this kind of distortion is brought about by varying just one parameter, $\gamma \cdot \gamma$ represents the angle by which the line dividing the given square domain at the centre, along the vertical axis, is twisted. Once that is achieved, the rest of the nodes in the mesh are shifted only along the horizontal axis with the vertical centre line as reference. This is done to achieve the kind of mix of skewed elements and bad aspect ratio elements in the same mesh, as was mentioned earlier. Once the mesh has been distorted the next step would be to use KLE to solve a test problem. The canonical test problem of semi-infinite region of stationary fluid bounded by an infinite horizontal flat plate given a sudden velocity along its plane is used here. This problem is discussed in some detail in the next section.

\subsubsection{The canonical test problem}

The tests were conducted on the well-known problem of a semi-infinite region of stationary fluid bounded by an infinite horizontal flat plate at $y=0$, which is suddenly given a velocity $U$ in its own plane and thereafter maintained at that speed. This problem has an exact analytic solution (see (22), Sec.4.3, among others). The velocity field described in a frame of reference fixed to a plate moving in the negetive $x$ direction is

$$
u(y, t)=U \operatorname{erf}\left(\frac{y}{\sqrt{4 \nu t}}\right)
$$


where erf is the error function and $y$ is the vertical coordinate. Rewriting (5.1) in terms of the normalized velocity $u / U$, the normalized vertical coordinate $y / Y$, and the parameter $\tau=\sqrt{4 \nu t} / Y$

$$
\frac{u}{U}=\operatorname{erf}\left(\frac{y / Y}{\tau}\right)
$$

where $Y$ is the height of the test mesh. The normalized vorticity distribution for this incompressible flow is given by the Gaussian function

$$
\frac{\omega}{U / Y}=\frac{2}{\tau \sqrt{\pi}} e^{-\left(\frac{y / Y}{\tau}\right)^{2}}
$$

This problem is closely related with the key process of $(\boldsymbol{\omega}, \boldsymbol{v})$ methods, i.e. the vorticity generation at a solid surface due to the induced slip and its further propagation to the body of the fluid. And in that sense, may be regarded as a canonical problem on the subject. For a specified time, the analytic solution for the velocity and vorticity fields are given, respectively, by the Gaussian and the error function of the spatial coordinate. The latter prevents the occurrence of the trivial case in which the analytic solution coincides exactly with any of the polynomial interpolant functions associated to the spectral-element technique. Thus, besides analyzing the time-dependant problem, the spatial analytic solution was used at specific times to test several aspects on the spatial discretization of the KLE. 


\subsubsection{The experiment}

This section briefly oulines the methodology for the controlled distortion experiment as well as the reasoning behind it. It then goes on to discuss the analysis of the results in order to come up with a suitable quality metric for the unstructured mesh being used to solve the KLE.

The first step was to solve the KLE on each distortion for different values of $h$ and $p$ and compare the results with the experimental values to get an estimate of the error. Here it is worthwhile to note that, when used inside the time-marching process of the vorticityvelocity scheme, the source term for the KLE solution at a given time is provided by a computation made by the ODE integrator from an approximation in weak form of the velocity field at the previous time step. This has the tendency to smooth out the shock introduced at the initial stage. Hence, forcing the theoretical vorticity distribution given by expression (5.3) at the initial stages as a source term for the KLE poses a very strict trial on the KLE solution. This sharp forcing is actually more challenging than KLE's normal operational requirements as the spatial counterpart in a vorticity-velocity scheme.

So for each distortion, both $h$ and $p$ were tried over a range of values keeping one constant while varying the other. As in spectral elements the nodes are not equidistant, the error in velocity was plotted against the number of intervals in each dimension $N^{\star}$, which is equal to the number of nodes minus one and represents the inverse of the average internodal dis- 
tance. To get a measure of what effect the distortion had on the solution both the velocity error and the condition number of the stiffness matrix were plotted against $N^{\star}$. Since the dominant flow is in the $\mathrm{x}$ direction, only the horizontal component of the velocity error was considered for the analysis. An infinity norm of the error was considered, to get a global estimate of the error in velocity. While the error gives an idea of the accuracy, the condition number tells us how stiff the matrix is, and how sensitive it is to perturbations. An ill-conditioned matrix not only converges very slowly but also blows up round-off and truncation errors which are to an extent unavoidable. These values were taken for successive values of $\tau$ at several stages of development of the boundary layer.

The problem considered here gives an initial jump in velocity, seen in the plots of the norm of the error vs $N^{*}$. For $\tau=0.01$ the error is two to four orders of magnitude greater than the error later on in the flow depending on the extent of refinement. Even though this is an important stage of flow, it is not ideally suited for the above mentioned experiment since the change in error with distortion is not very significant. This is expected since there is an initial shock at the beginning which dominates the error. Figure 5.2 shows plots of an error norm against $N^{*}$ for different values of $\tau$. Plots of velocity error against the order of discretization show that for $\tau$ values of 0.01 there is very little effect of increasing the order of discretization or even the distortion. For this purpose the plots of the error norm vs $N^{*}$ are observed for stages further into the development of the boundary layer. Therefore, for this experiment the plots for $\tau=0.2$ and $\tau=0.9$ are used. After 0.9 the domain truncation error seems to dominate, therefore the range of $\tau$ between 0.2 and 0.9 is quite reasonable. 


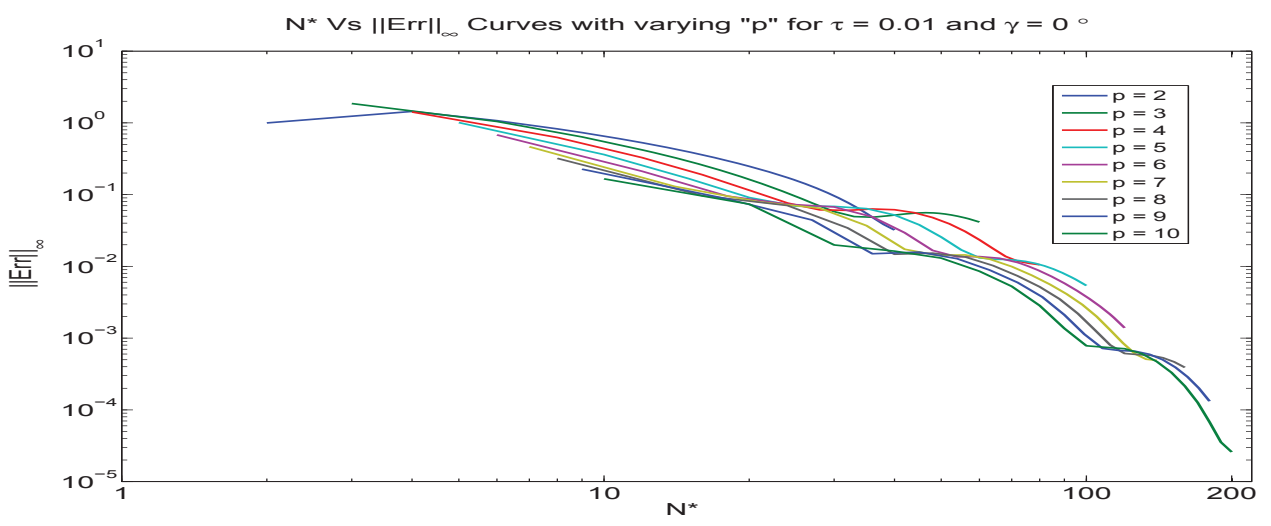

(a) $\gamma=0^{\circ}$

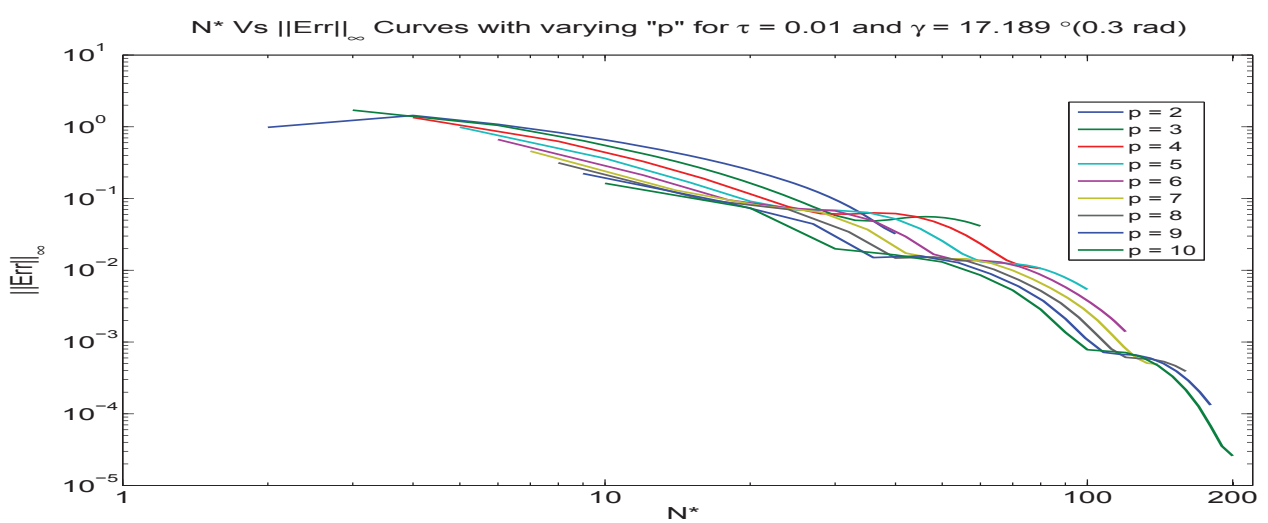

(b) $\gamma=17.2^{\circ}$

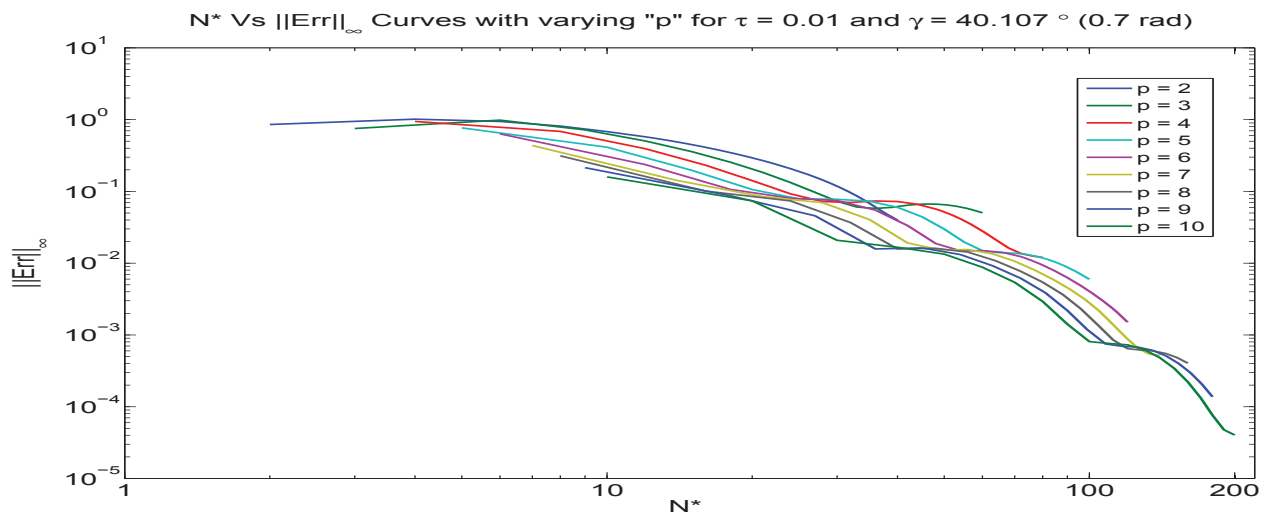

(c) $\gamma=40.1^{\circ}$

Figure 5.2: Error plots for initial stages of boundary layer development for three different distortion values while varying the order of the interpolation polynomial " $p$ " 


\subsection{Observations and analysis}

The analysis of the controlled distortion experiment can basically be broken down into two parts. In the first part, the various plots obtained for different distortions and varying $h$ and $p$ refinement are analyzed for the error. This is used to find the threshold distortion after which, as will be seen later, there is huge jump in error. The second part is then concerned with finding out a quality metric that can predict this jump in error, with reasonable dependability based on the geometrical aspects of the mesh elements.

\subsubsection{Error analysis}

The purpose of this stage of the experiment is to find a threshold value for distortion. The first step is to plot the condition number and the error norm of the velocity against $N^{*}$ for different values of $\gamma$. These plots are taken for both an $h$-refinement as well as a $p$ refinement, varying one while keeping the other constant. One must remember that the error can be reduced by the above-mentioned refinements, so the purpose of the above tests would be to give a threshold value of distortion by seeing how the error behaves with distortion and not the order of discretization. So if the same value of distortion gives similar results of an extremely high error and condition number, it should give the desired threshold value. Since the condition number is independent of the solution, depending only on the meshing, an agreement between the results obtained by plots for the condition 
number and the error norm for varying discretization should validate the threshold value observed. Figure 5.3 shows plots of the condition number against $N^{*}$ keeping the NGL constant i.e the $h$-refinement plots for the condition number. The plots clearly show that the condition number shoots up for $\gamma=0.75$ radians, so the threshold value must lie below the 0.75 radians value, somewhere around 0.7 radians where it shows reasonable values. A check on the $p$-refinement curve shows similar results, as can be seen in the plot shown for meshes with 7 and 20 elements respectively along a given direction.owing to such high values the problem infact was not converging for values even slightly more than 0.75 . As can be seen that the condition number is already going up at around $0.7 \mathrm{rad}$, it was decided to limit the distortion to $0.7 \mathrm{rad}$, and this was justified by the error plots.

The next set of plots in Figure 5.4 are for infinity norm of error in velocity Vs $N^{*}$ for both $h$ and $p$-refinement. Keeping in mind the value of $\gamma$ obtained from the condition number plots, values for error for $\gamma$ varying from 0 to 0.7 radians were plotted against $N^{*}$, again for both $h$ and $p$-refinement. As mentioned earlier these values are taken for $\tau=0.2$ and $\tau=0.9$. These plots clearly show how the error blows up for $\gamma=0.7$ radians. The log values of the norm of error are taken since it gives a clearer picture of how and when the error shoots up. The difference between the error values are apparent for the two different cases of $\tau$, since with increasing $\tau$ the boundary layer stabelizes further, as opposed to the intial discontinuity. The three plots shown here are part of a series of plots for an interpolation order ranging from 2 till order 10 and the number of elements ranging from 2 all the way till 50. This was, however, done keeping in mind the computational power 


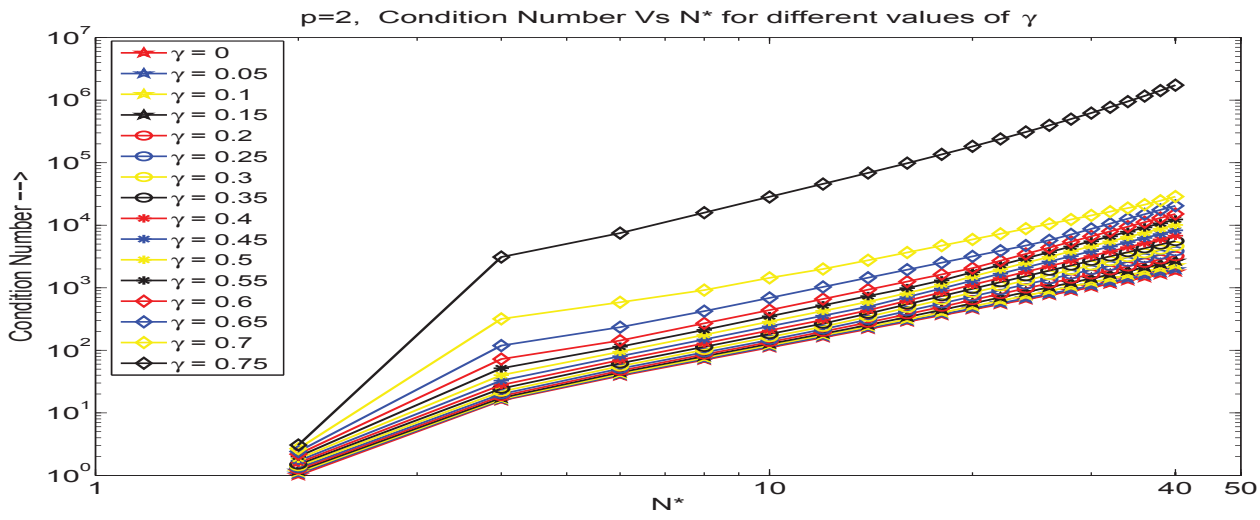

(a) Interpolation order $p=2$

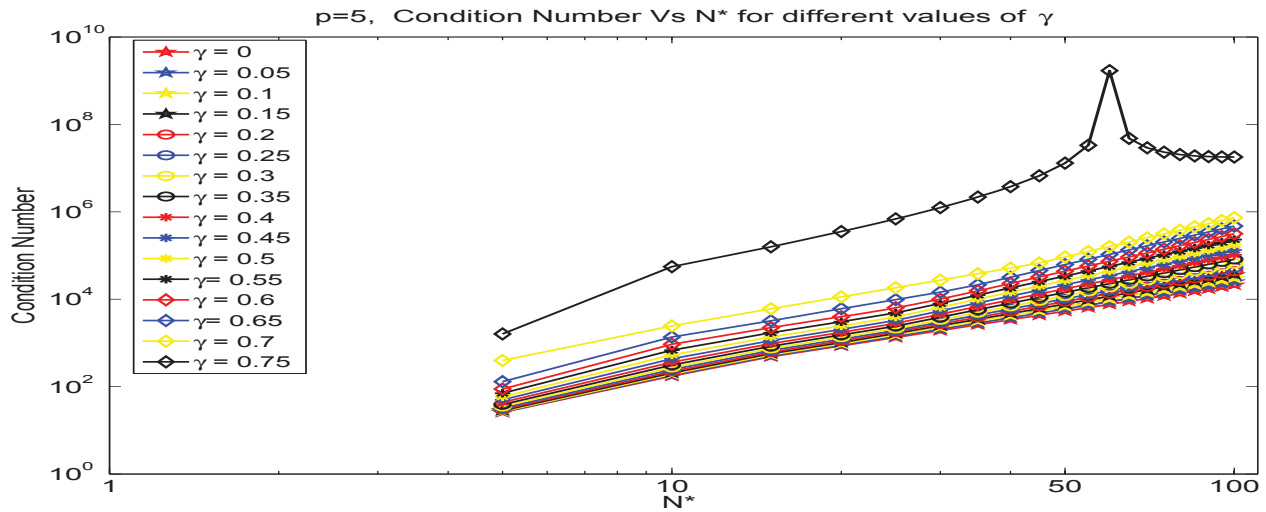

(b) Interpolation order $p=5$

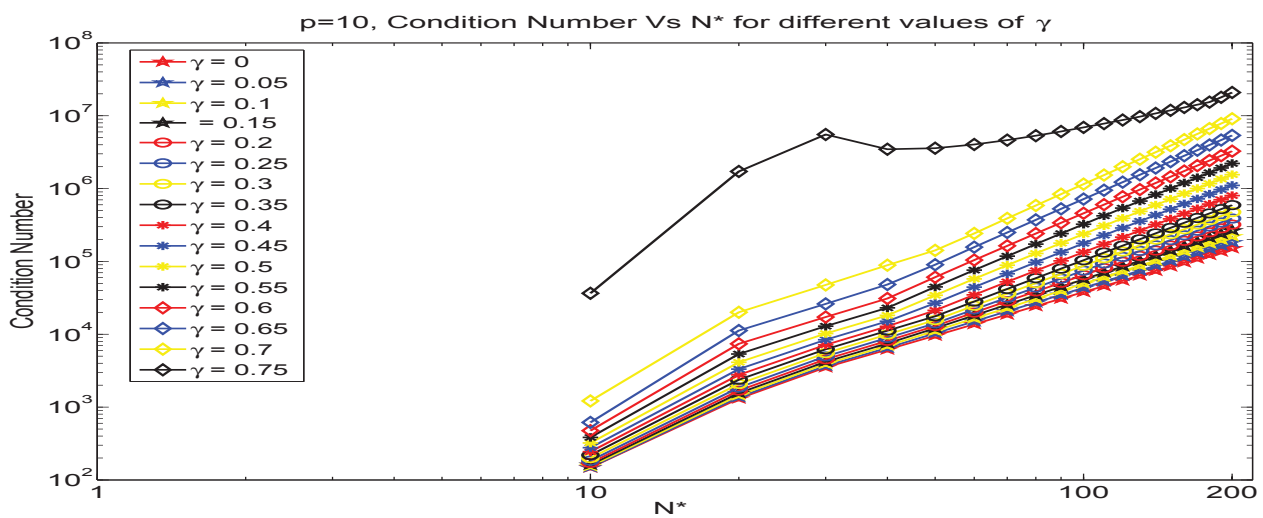

(c) Interpolation order $p=10$

Figure 5.3: Plots showing how the condition number increases with increase in mesh distortion, keeping the interpolation order " $p$ " constant, such that $\gamma$ is measured in radians 


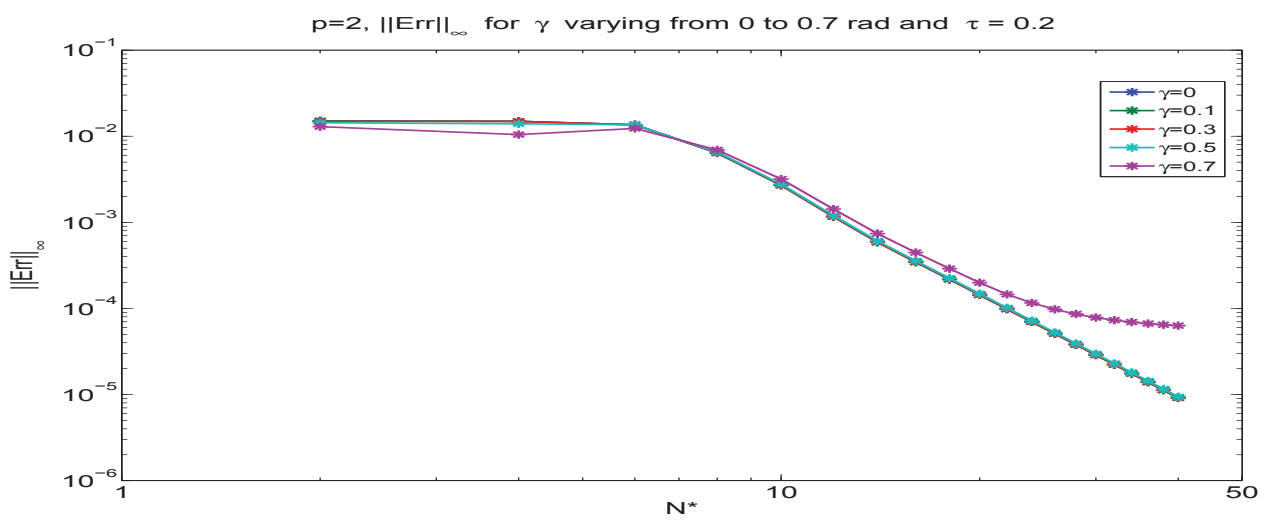

(a) $p=2$ and $\tau=0.2$

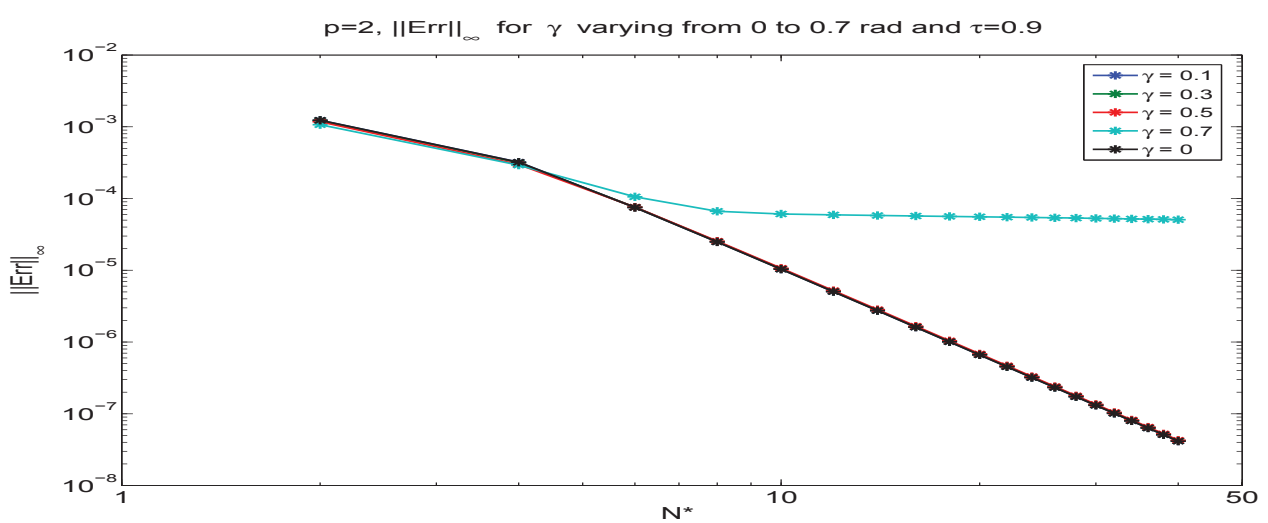

(b) $p=2$ and $\tau=0.9$

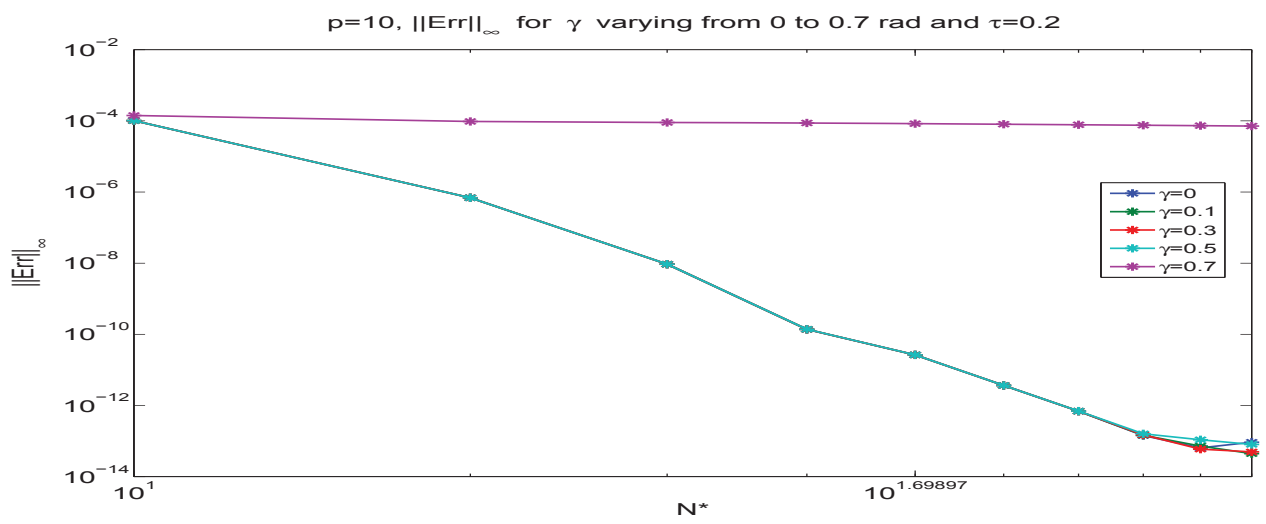

(c) $p=10$ and $\tau=0.2$

Figure 5.4: Plots of the error norm for different values of $\gamma$, keeping $p$ constant and varying the number of elements $h$, for $\tau=0.2$, where $\gamma$ is given in radians 
available. Hence the values were selected to ensure that the $N^{*}$ values did not go beyond 320. This was based on an earlier experiment performed to test the computational limit of the available resources. In fact there was no real need to go beyond 250 since after that the error values did not change much and an increasing round off error more or less took over from the decreasing truncation error. An important observation from the plots (a) and (c) of Figure 5.4 shows how a higher value of $p$ gives more accurate solutions even for the initial stages, for relatively lesser number of elements.

The next step was to locate more precisely the threshold value of distortion. Figures 5.55.6 represent plots taken over a range of $h$ and $p$ refinements with varying gamma and two values of $\tau$ equal to 0.2 and 0.9 . The $\gamma$ values are increased from 0.6 to 0.75 radians. The increment in $\gamma$ was performed over a range of step sizes and finally a size of 0.0125 was observed to give satisfactory results. Plots for NGL values varying from 3 to 11 were taken. This was to ensure that the threshold was based on the distortion rather than just the h or p refinement. The different plots show a range of the threshold value varying from somewhere around 0.61 to 0.68 radians. A combined plot for all these values given in Figure 5.5 for both $\tau$ clearly shows this. 

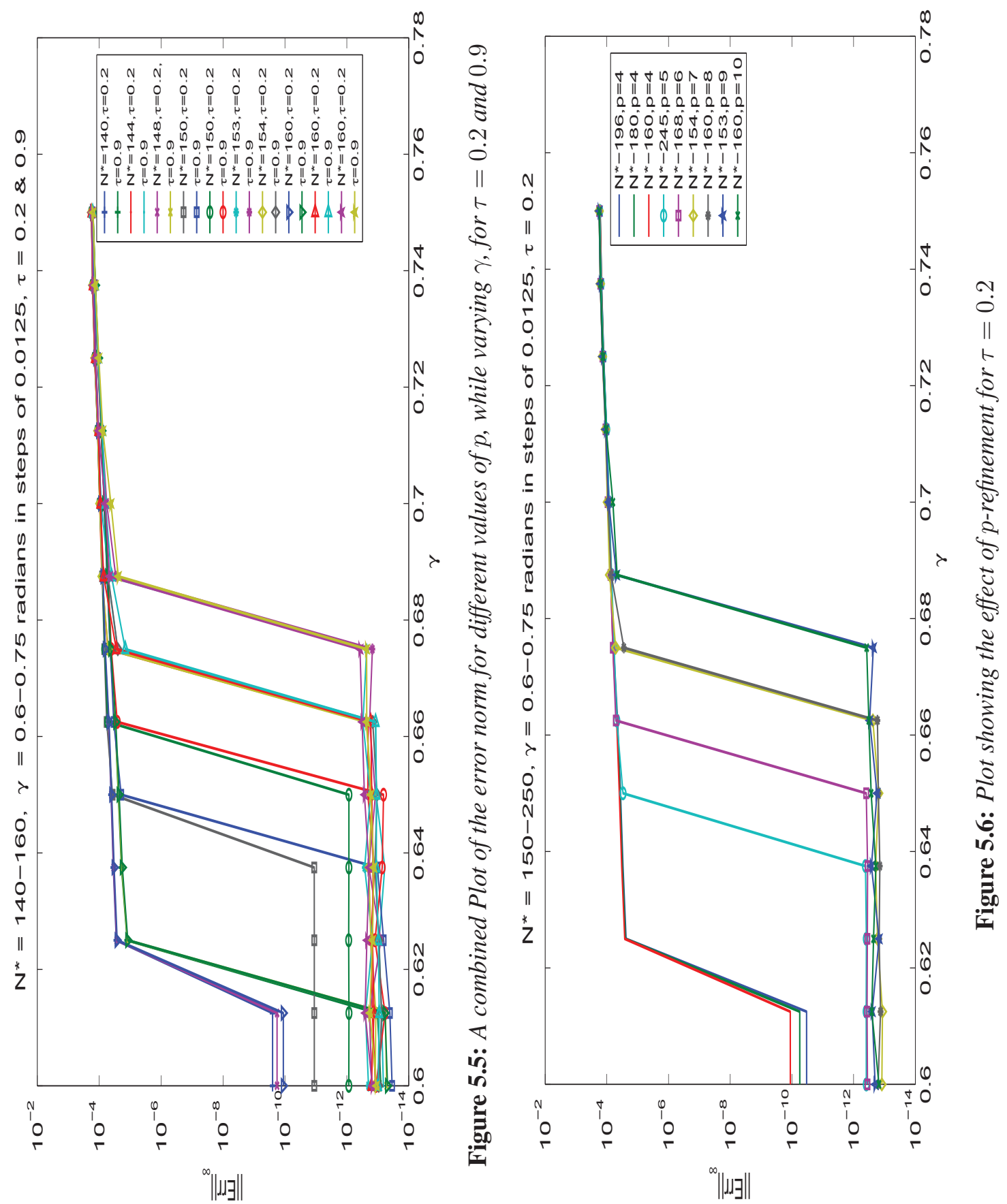
The discretization has been represented by the $N^{*}$ value to avoid confusion. This plot just shows that the threshold value lies approximately in the region between 0.61 to 0.68 radians, but an interesting result can be seen by observing the plot in figure (5.6) for tau=0.2 alone. It can be seen that by increasing the value of NGL by just one order, the error can be brought down dramatically, even when the number of elements is decreased by almost half. This reduction in the $N^{*}$ value has a huge effect on the computational power required and is one important advantage of the spectral element methods.

So it is safe to assume that a distortion of over 0.68 radians is enough to cause the KLE to fail no matter what the mesh refinement value is. Once the threshold has been decided upon we can go on to see how well the various quality metrics mentioned above represent the distortion, especially for the region between 0.61 to 0.68 radians depending upon the refinement being considered.

\subsubsection{Quality Metric}

This section analyzes the effect of distortion on the mesh using different quality metrics in order to select the most suitable metric. The table (5.1) shown in the next page lists the various quality metrics for both triangular and quadrilateral elements that were finalized for the test. The distortion was carried out by varying $\gamma$ as defined in Figure 5.1. The quality plot was then compared with the corresponding error plots, i.e for the plot with similar 
Table 5.1

Quality metrics used for a 2-D distorted element

\begin{tabular}{|c|c|c|}
\hline Quality type & Quadrilateral & Triangle \\
\hline $\begin{array}{l}\text { Quadrilateral shape met- } \\
\text { ric (39) } Q_{Q U A D}\end{array}$ & $\begin{array}{l}\frac{8}{\sum_{k=0}^{3}\left(1+r_{k}^{2}\right) /\left(r_{k} \sin \theta\right)} \\
\text { where } r=\sqrt{\gamma_{22} / \gamma_{11}} \text { is the } \\
\text { length ratio such that } \gamma_{11}^{k} \text { is } \\
\text { the length of the side con- } \\
\text { necting nodes } k \text { and } k+1 \\
\text { and } \gamma_{22}^{k} \text { is the length of the } \\
\text { side connecting nodes } k \text { and } \\
k+3\end{array}$ & NA \\
\hline Diagonal Ratio (40) $Q_{D R}$ & $\begin{array}{l}\boldsymbol{\operatorname { m a x }}\left[\boldsymbol{d}_{1}, \boldsymbol{d}_{2}\right] / \boldsymbol{\operatorname { m i n }}\left[\boldsymbol{d}_{1}, \boldsymbol{d}_{\mathbf{2}}\right] \\
\text { where } d_{1} \text { and } d_{2} \text { are the } 2 \text { di- } \\
\text { agonals of the quadrilateral }\end{array}$ & NA \\
\hline $\begin{array}{l}\text { EquiAngle Skew (40) } \\
Q_{E A S}\end{array}$ & $\begin{array}{l}\max \left[\frac{\theta_{\max }-90}{180-90}, \frac{90-\theta_{\min }}{90}\right], \\
\text { where } \theta_{\max } \text { and } \theta_{\min } \text { are } \\
\text { the maximum and minimum } \\
\text { angles (in degrees) between } \\
\text { the edges of the element }\end{array}$ & $\begin{array}{l}\max \left[\frac{\theta_{\max }-60}{180-60}, \frac{60-\theta_{\min }}{60}\right] \\
\text { where } \theta_{\max } \text { and } \theta_{\min } \text { are } \\
\text { the maximum and mini- } \\
\text { mum angles (in degrees) } \\
\text { between the edges of the } \\
\text { element }\end{array}$ \\
\hline $\begin{array}{l}\text { MidAngle Skew } \\
Q_{M S K W}\end{array}$ & $\begin{array}{l}\cos \left(\theta_{\min }\right) \text {, } \\
\text { where } \theta_{\text {min }} \text { is the minimum } \\
\text { angle formed between the } \\
\text { bisectors of the edges of the } \\
\text { quadrilateral }\end{array}$ & NA \\
\hline
\end{tabular}


number of elements and value of $\gamma$ to relate the threshold value of $\gamma$ to the worsening quality. The quality metrics used are based on shape measures as well as skew metrics. The basic idea of a shape measure is that it relates both skew as well as the aspect ratio to the element quality (39). This is not always desirable since the aspect ratio measure is not well suited for an unstructured quadrilateral mesh which can have very extreme edge sizes and angles (38). In fact, while using the meshing algorithm it was ensured that the element sizes and aspect ratios were kept in control. And since the triangle was divided into three quadrilaterals using the centroid, the sizes obtained where comparable. Thus the main purpose of this experiment is to check the distrotion for skewness, hence the predilection towards skew metrics. It should be noted that there is no real control over the quadrilateralization, as far as the skewness is concerned, so the quality metric is the only way an element can be analyzed for iteration till an acceptable quality is obtained. The following figures show a color map representing quality of each element in terms of the metric being used. Each figure consists of two color maps each for different levels of distortion. Those for the Diagonal ratio, Equi-angle skew and Mid-angle skew quality metrics consist of two figures, one before and one after the threshold value. Here $\gamma$ varies from 0.4 to 0.65 radians and the number of elements along an axis for all these figures is 6. The $\gamma$ values shown for the quadrilateral shape metric in Figure 5.8 are both before the threshold value to basically give an idea of how this metric is not at all suited for the problem at hand. Figure 5.7 clearly shows how the $\mathrm{Q}_{d r}$ metric fails for higher distortion. With increasing distortion the most skewed elements all of a sudden have better qualities 

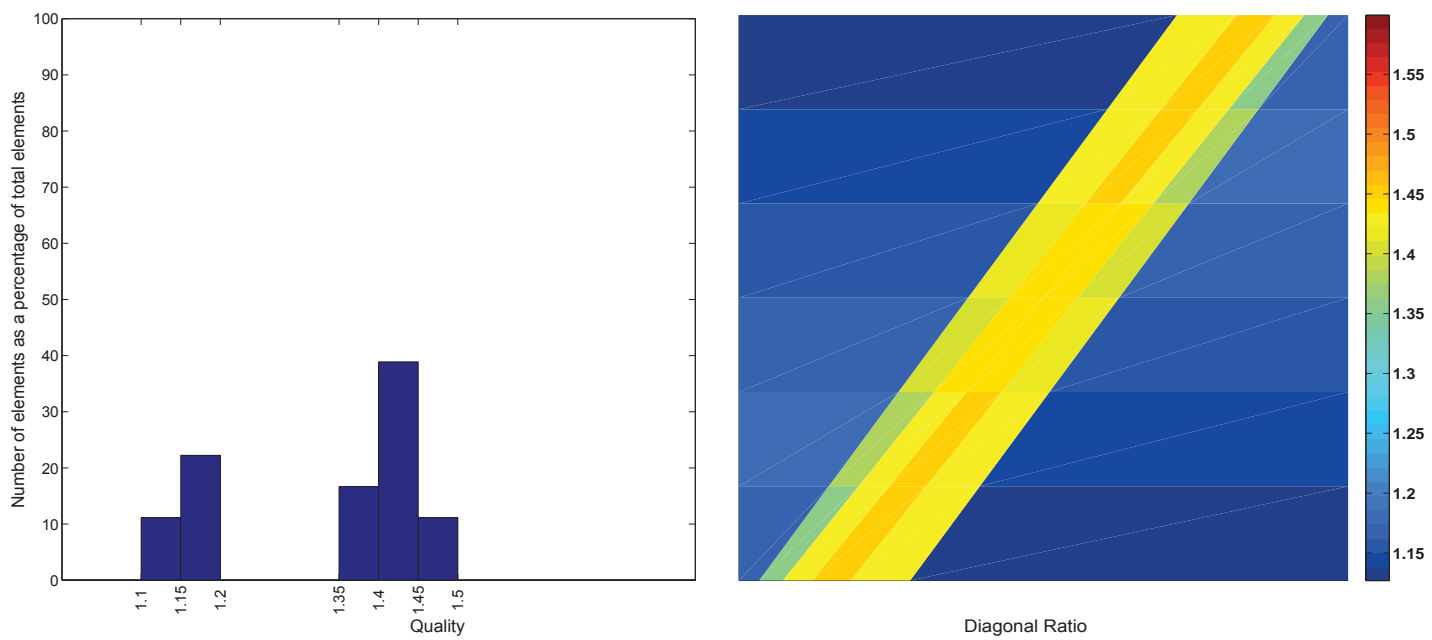

(a)
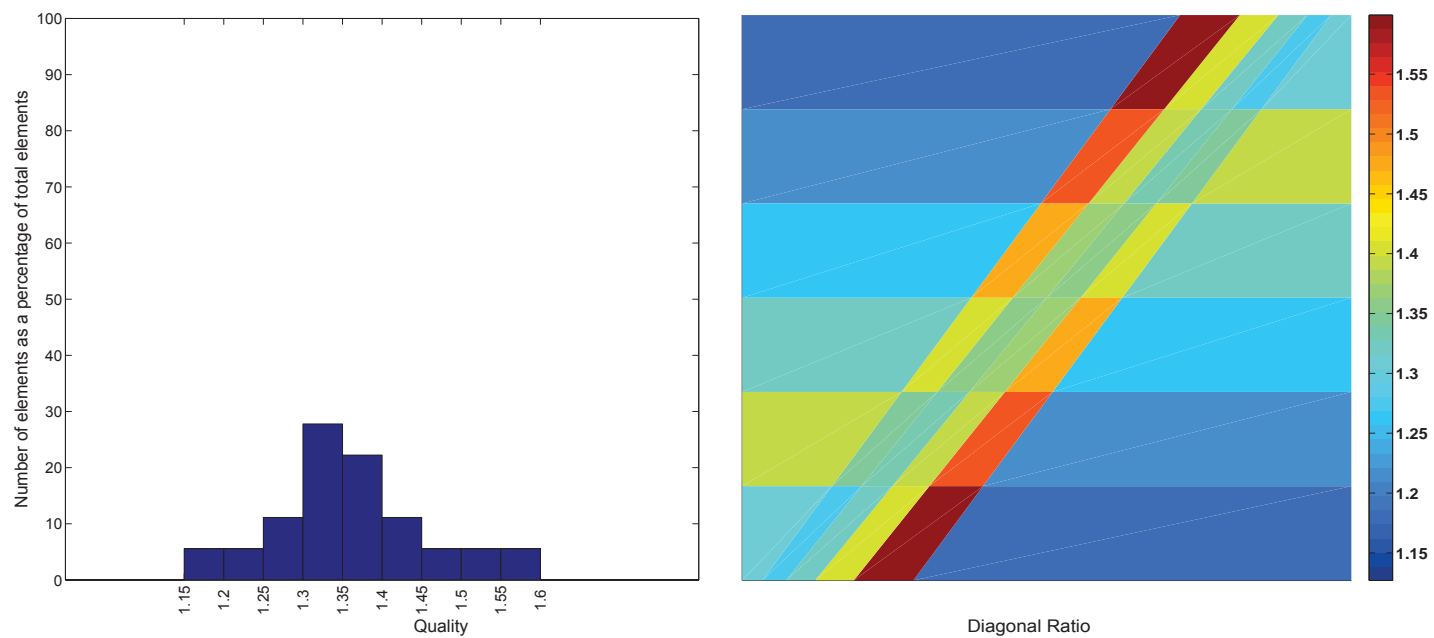

(b)

Figure 5.7: A color-map and a histogram representing mesh quality Using the Diagonal Ratio quality metric for two meshes with different levels of distortion

than the less distorted elements and the quality for the most distorted elements seems to be increasing with increasing distortion. This can also be seen in the histograms for this quality metric, where the percentage of elements with really bad qualities has actually gone down, despite the fact that the quality of some of the elements has worsened. So this is clearly not the metric which can be used for this case. 
The $\mathrm{Q}_{q}$ quality measure represented by a color map in Figure 5.8 shows better results as far as worsening quality conditions with increasing distortion are concerned. But, as mentioned before, this measure is a blend of aspect ratio and skewness, which can clearly be seen in the plots. The quadrilateral elements which seem to be of decent quality as far as the skewness is concerned turn out to give bad quality values. The worst quality elements are clearly the ones with both skewness and bad aspect ratios rather than just the most skewed elements. The remaining two quality metrics are based entirely on a

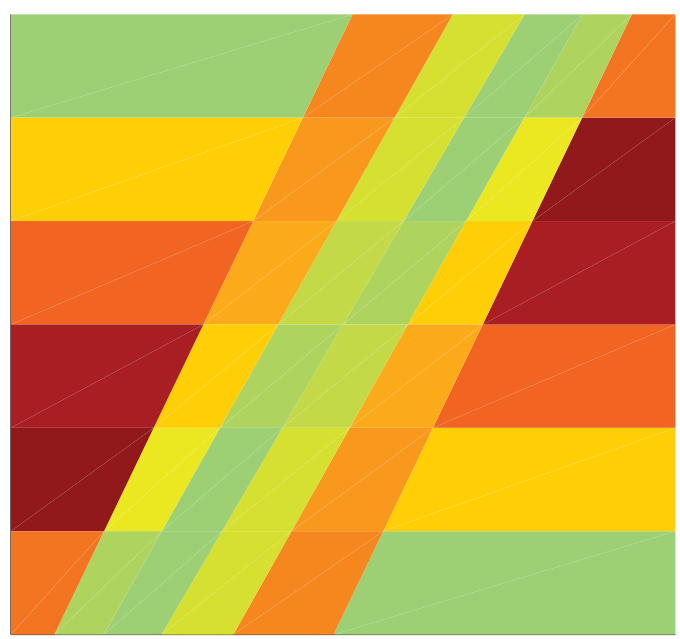

(a) Nel=6 and $\gamma=0.4$ radians

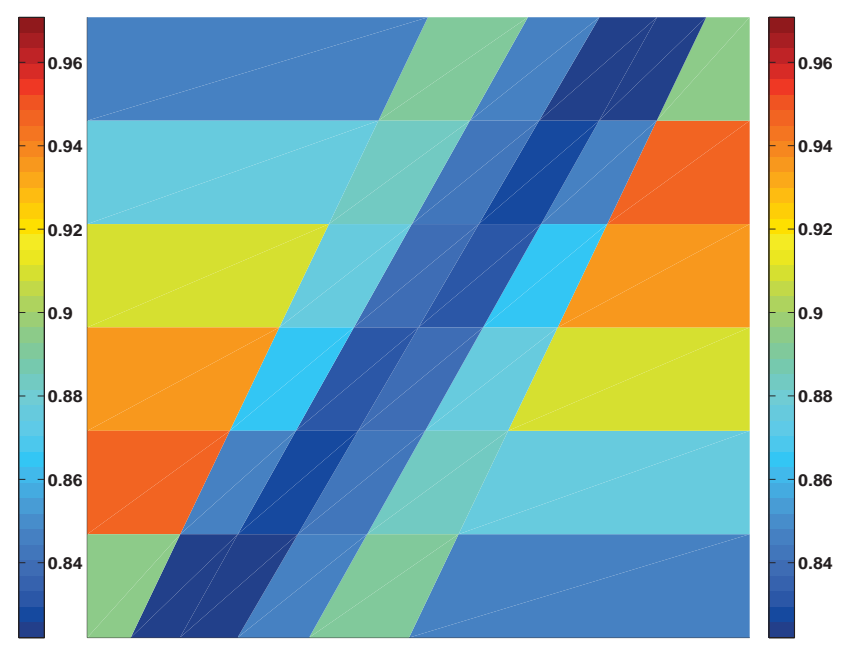

(b) $\mathrm{Nel}=6$ and $\gamma=0.5$ radians

Figure 5.8: A color-map representing mesh quality using the Quadrilateral shape metric for two meshes with the same number of elements but increasing distortion

normalized skewness value of the elements. They show similar results as can be seen in the plots shown in Figures 5.9 and 5.10. The elements with maximum distortion in angle are the worst elements. This agrees well with the error plots, so seem to be best suited for this case. The histograms also clearly show a shift in the quality values towards a region with poorer quality, as the distortion increases. The $\mathrm{Q}_{\text {eas }}$ quality metric (Figure 5.10)does 

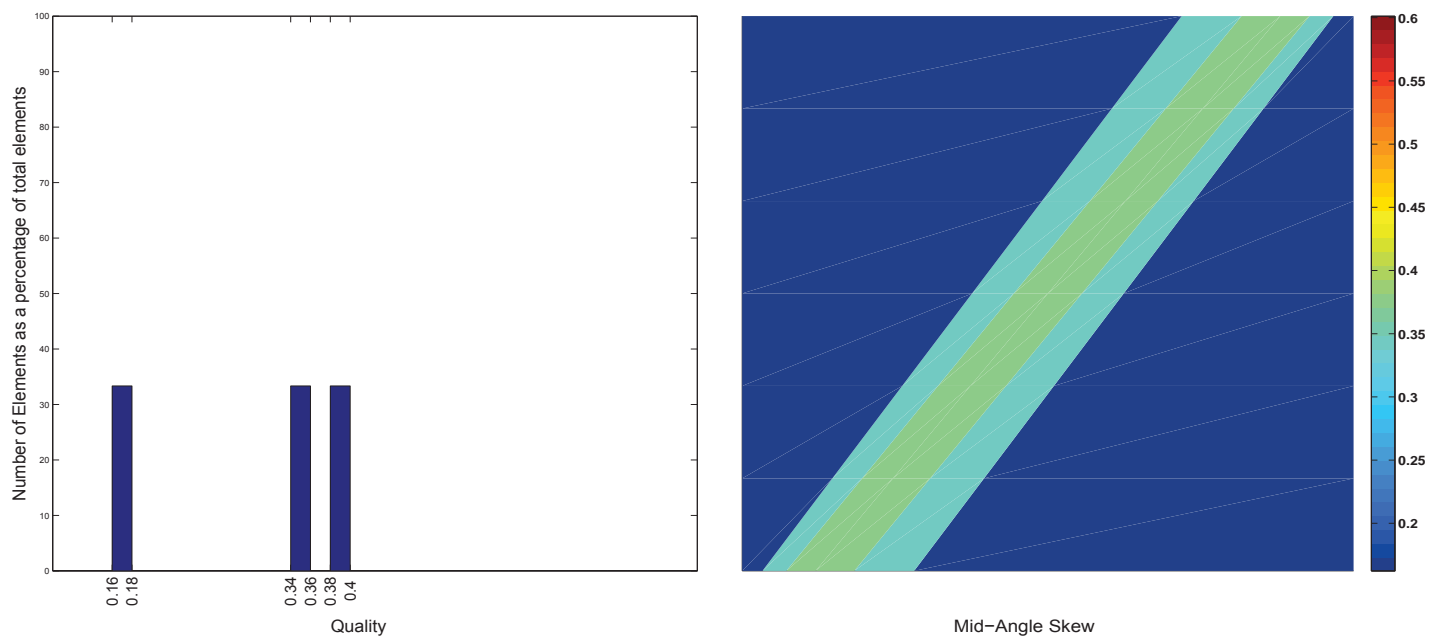

(a)
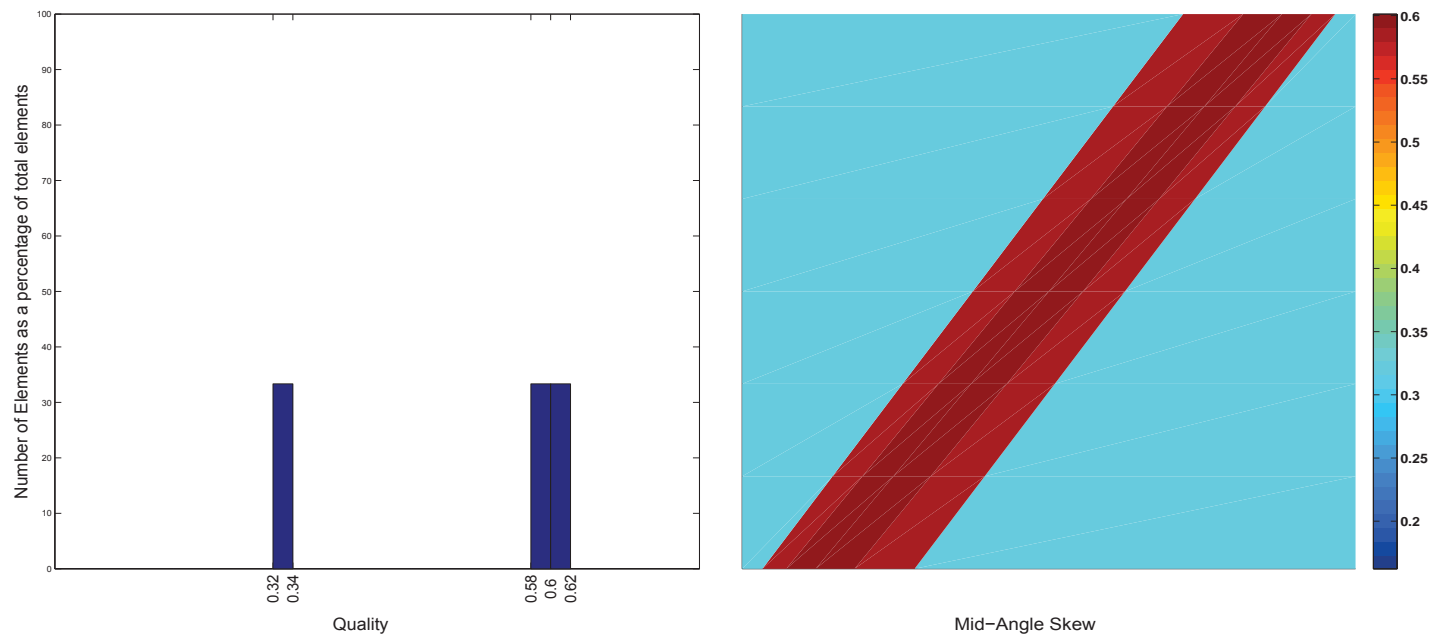

(b)

Figure 5.9: The Mid-angle skew quality metric for two meshes with the same number of elements but varying distortion

seem to be more sensitive to the distortion, i.e. the skewness as compared to the $\mathbf{Q}_{m s k w}$ (Figure 5.9). Apart from that, it is easier to implement as far as the computational effort is concerned. So $\mathrm{Q}_{\text {eas }}$ is the obvious choice for this particular case. This quality metric $\mathrm{Q}_{\text {eas }}$ was then used to check the quality of the unstructured tri-quadrilateral mesh being used for the KLE. A number of tests were then performed for different distortions, with 

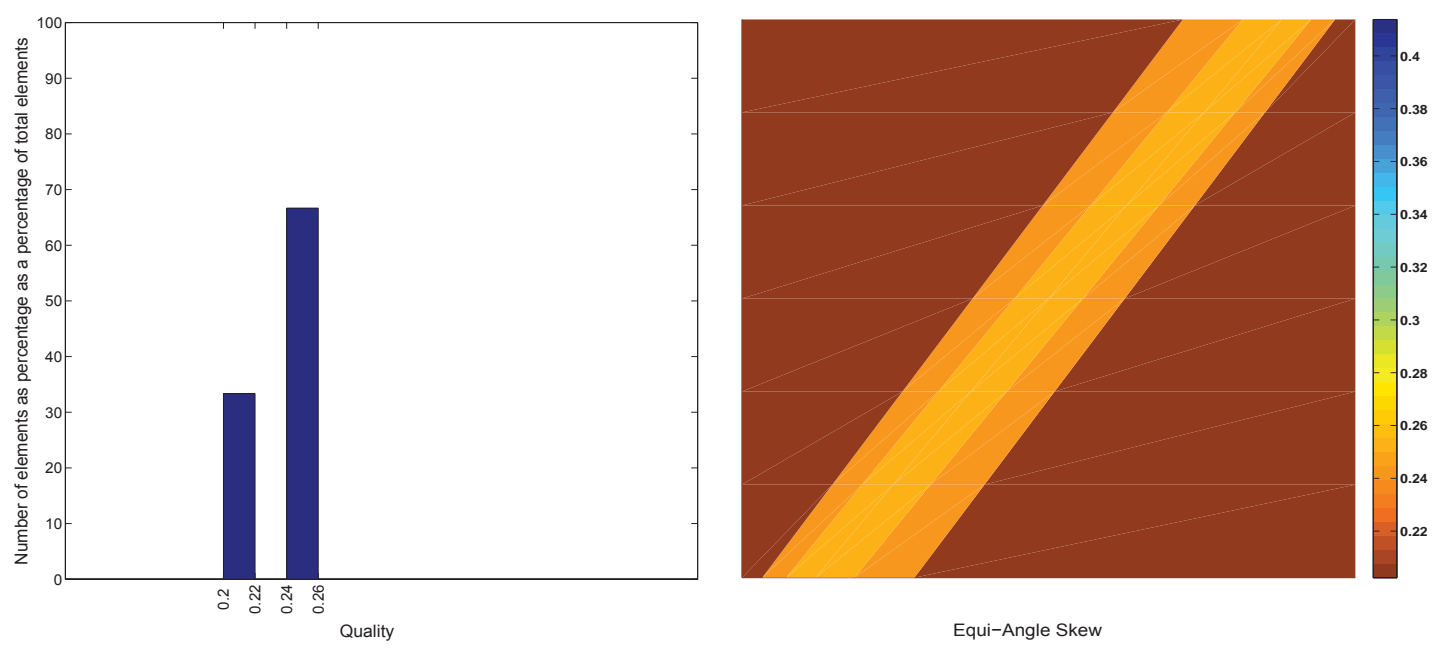

(a)
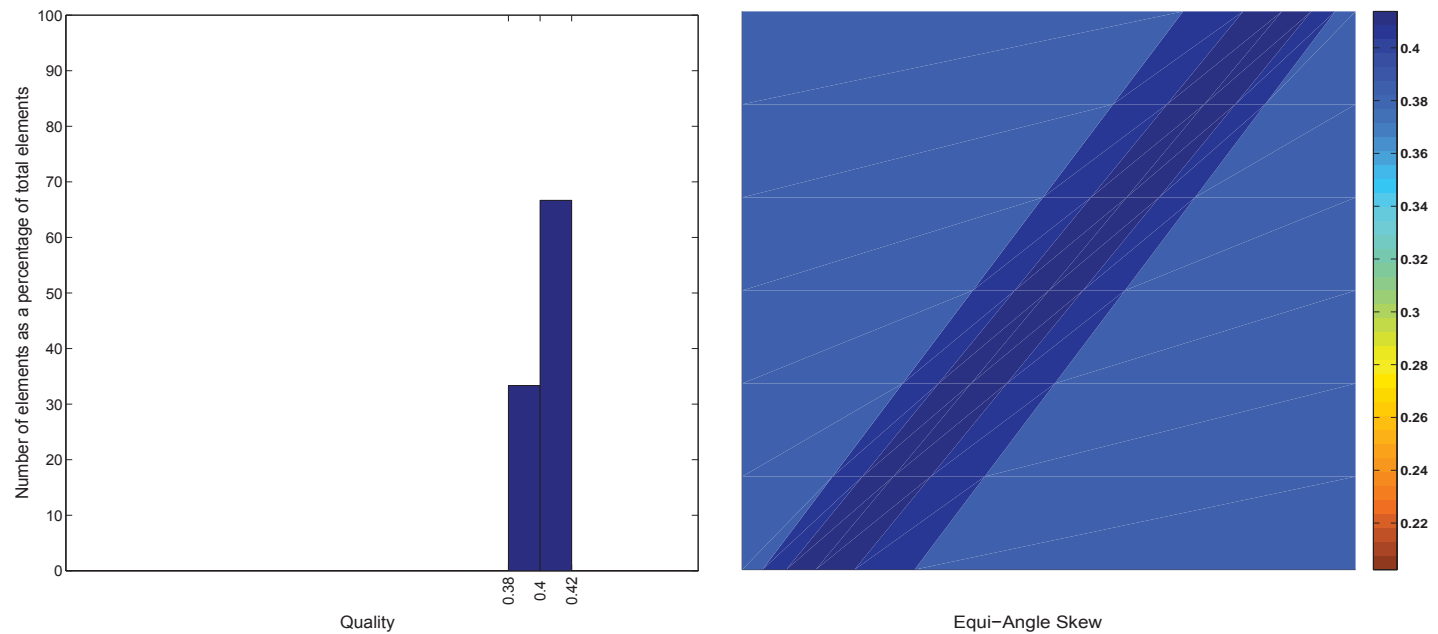

(b)

Figure 5.10: The Equi-angle skew quality metric for two meshes with the same number of elements but varying distortion

different levels mesh density to find a relation between the mesh quality using $\mathrm{Q}_{e a s}$ and the threshold value of distortion. It could be seen from these results that every time an element with quality greater than 0.42 was found, a substantial jump in error could be seen.

Figure 5.11 is an example of how an unstructured tri-quadrilateral mesh looks like along with a color-map representing the quality of that mesh. The elements showing the worst 


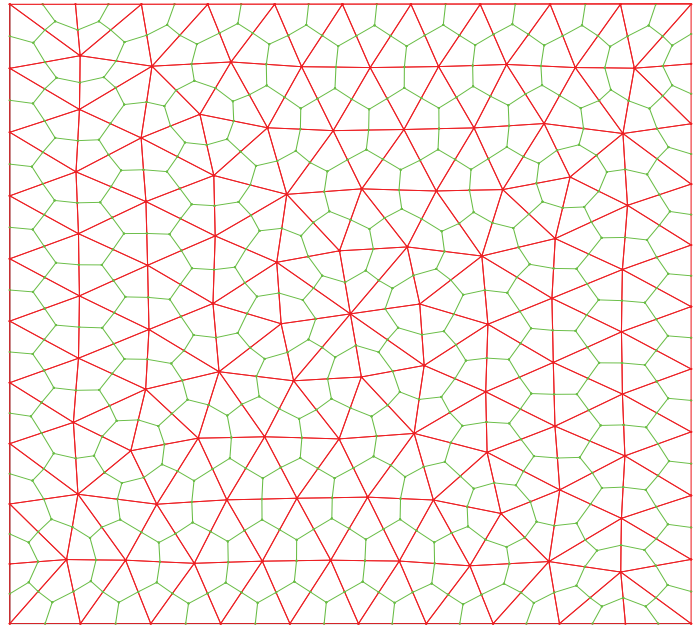

(a) A tri-quadrilateral Mesh with 21 Edge nodes

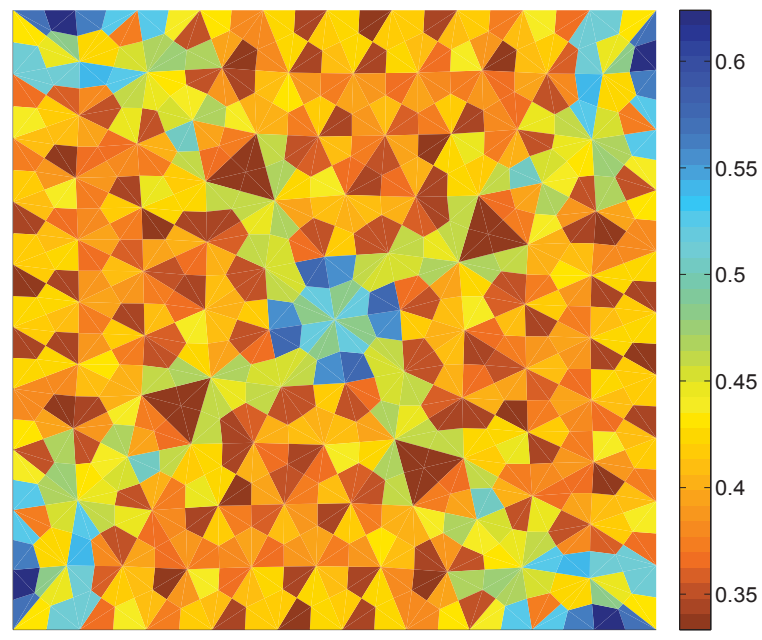

(b) A color-Map Showing the quality of the triquadrilateral elements Using the $Q_{\text {eas }}$ metric

Figure 5.11: A tri-quadrilateral Mesh for a Square plate along with a color-Map for qaulity using the Equi-anle Skew quality Metric

quality, comparable with those for worst distortions of the controlled distortion experiment, were observed. These are the ones with the darkest shade of blue. It was noted that the elements with the maximum obtuse angle at the centroid of the triangles showed the worst qualities. This can be checked again with a more complicated geometry than a square plate as shown in Figure 5.12. This geometry consists of a rectangular domain with a cylindrical region in between and a much denser mesh than the previous example. Figure 5.13 shows a color map for quality for this mesh. A zoomed in region of the color map has also been shown, where the region highlighted by a red square shows the element whose quality is poor because of an angle greater than $120^{\circ}$. 

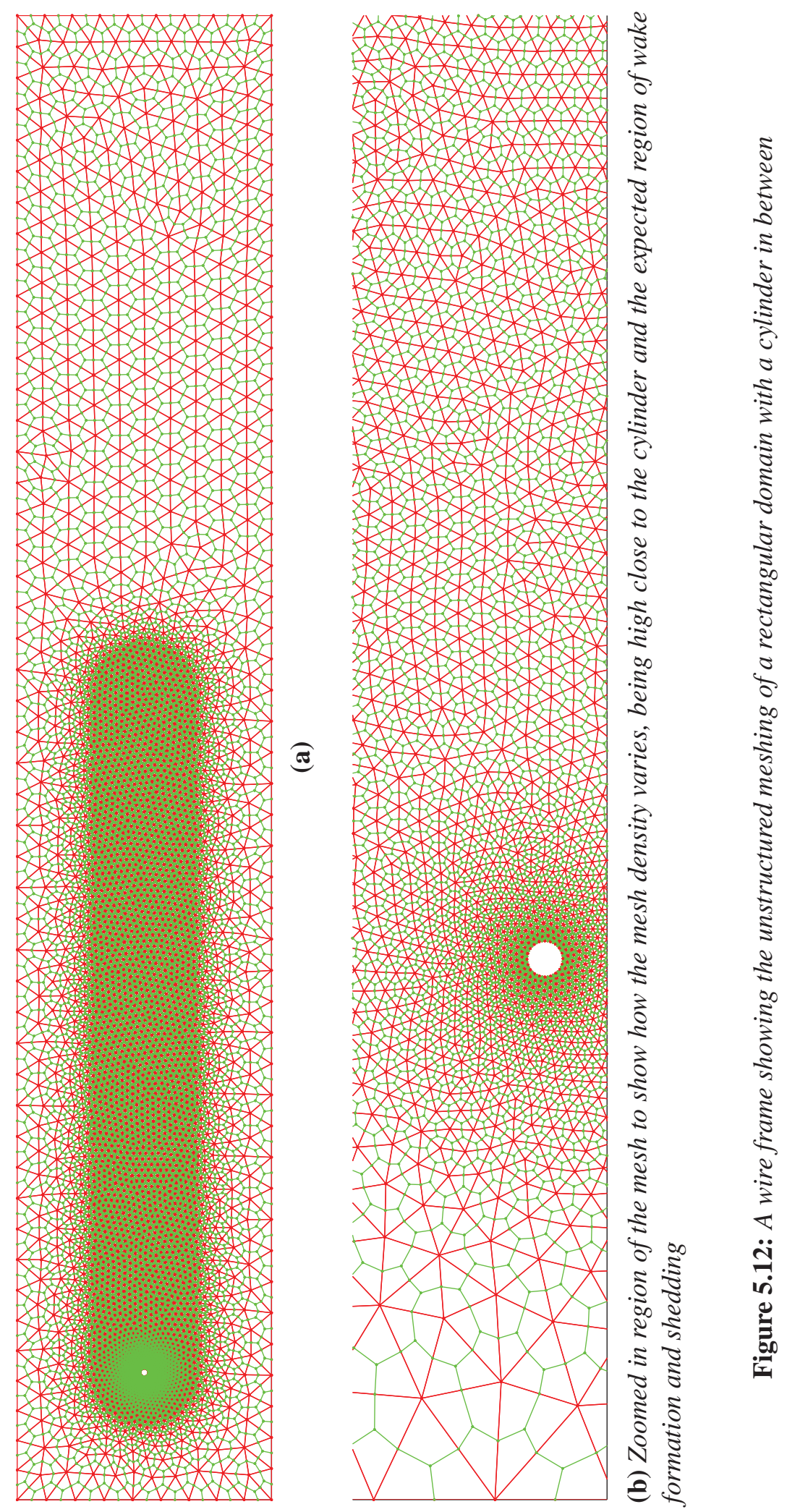

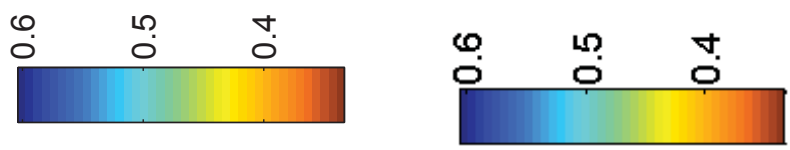

$\frac{5}{2}$
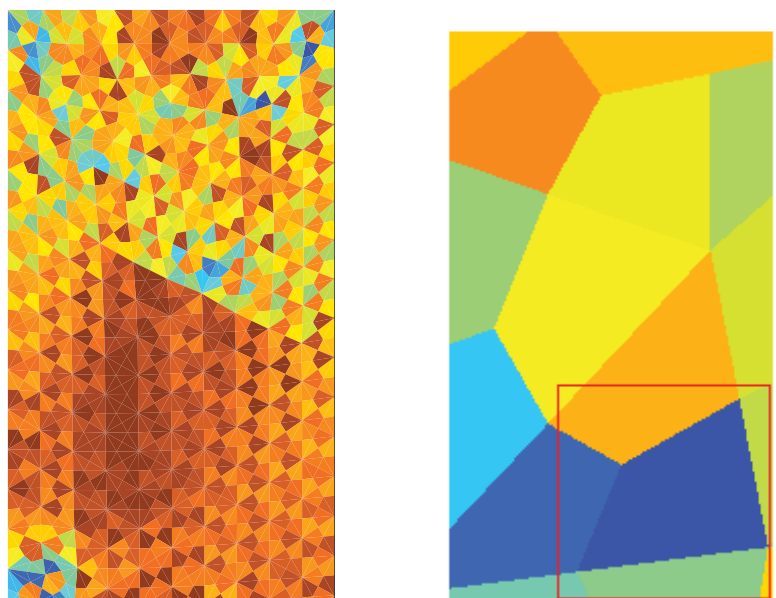

है

1,1

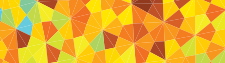
$-814$

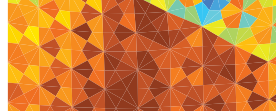

18

18

48

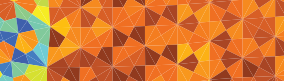

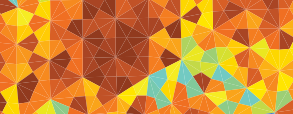

- t.

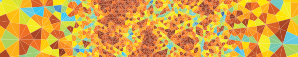

త్ర

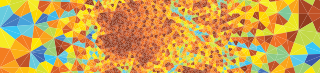

\&.3.5.t.

$\neg \ldots y^{2}+x^{2}=1$

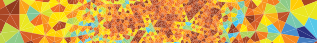

p.r.t.

$\because .510$

iv 110

- bithrition

a. 310

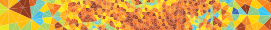

4315

- $450 .+2$

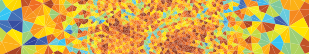

$10+5$

wh o : ?

a

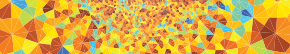

$1+\cdots 0$

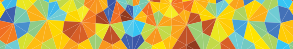

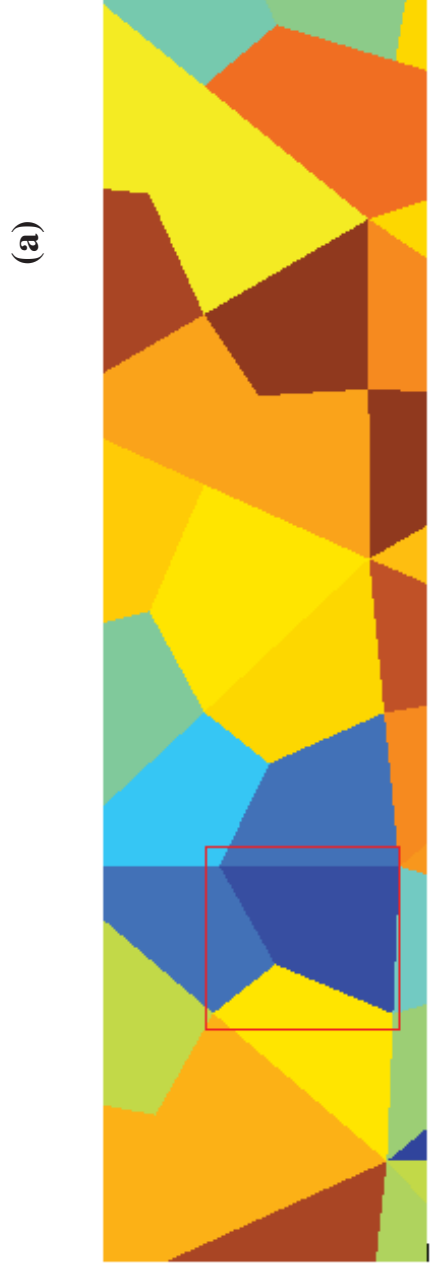

$\frac{\sqrt{2}}{\frac{2}{2}} \cdot \frac{2}{2}$

$\frac{2}{3}$

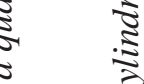

० ठ

:

ป

ป

क $\frac{5}{3}$

ป $\quad$ ป

ป ?

๘

शे है

ธิ च

离

ป

3.

$\frac{2}{3}$

$\frac{3}{4} \quad \frac{0}{0}$

की

है व

\&

ठ के

ईิ है

ฐ

छ)

읨

อ

茯 


\subsection{Improved tri-quadrilateralization}

The triangulation was designed to take care of thin elements, thereby reducing the possibilty of exrtemely acute angles. But the angle formed at the Steiner point (the centroid in our case) was not controlled in any way. So it was decided to ensure an angle of $120^{\circ}$ at the center to keep the error manageable. This was done by changing the location of the Steiner point from the centroid to the Fermat point of the mid-point triangle of each triangle. Mathematically, the Fermat point of a triangle solves a Steiner tree problem to minimize the sum of distances of a point from all three vertices. The method used in this research is based on a paper by Anthony Varilly (41). This procedure fails for triangles with a maximum angle greater than $120^{\circ}$. For such cases, the Steiner point was left at the centroid. However, it is worth mentioning here, that the algorithm used for the triangulation, has, for all the geometries used in the test, not given a single triangle with any of the angles greater than $120^{\circ}$. The formula used for the Fermat point also fails for equilateral triangles, in which case the Fermat point coincides with the centroid. Following is the formula used for obtaining the Fermat point

$$
\begin{array}{r}
u=\left(\sqrt{3} b c-\sqrt{3} a^{2}-a c-a b\right)(b-c) \\
v=\left(a^{2}+\sqrt{3} a b+\sqrt{3} a c+3 c b\right)(b+c) \\
d=2 \sqrt{3}\left(a^{2}+b^{2}+c^{2}\right)+6 a c+6 a b+2 \sqrt{3} b c \\
T=\left(\frac{u}{d}, \frac{v}{d}\right)
\end{array}
$$


Where, $a, b, c$ are as shown in Figure 5.14 and $T$ gives the location of the Fermat point. Applying the above equation to the triangles in the initial mesh gives us a tri-quadrilateral

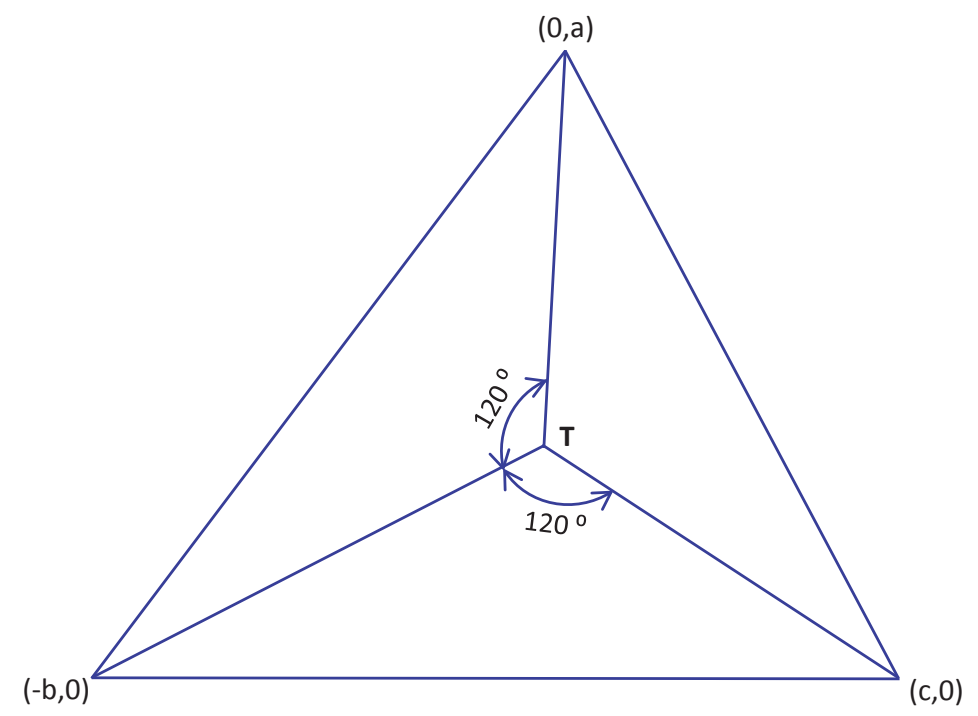

Figure 5.14: A triangle displaying the variables used in the above equation

mesh with considerably better quality as can be seen from Figure 5.15 which uses histograms and color maps to compare the two meshes, i.e. the one using the centroid and the other the Fermat point to achieve the tri-quadrilateralization.

It is easy to see from the histogram that the percentage of higher quality elements goes up dramatically for a tri-quadrilateral mesh using the Fermat point in place of the centroid to achieve the quadrilateralization. This can also be seen in the color-map where the map for the mesh using the Fermat point has a lot more elements in the dark red region, which happens to represent the good quality elements. The figures shown from page 90 onwards are used to reiterate the above-mentioned observation, for a couple of meshes denser and more complex then the meshes used thus far. From the Figures 5.16-5.19, it can be 

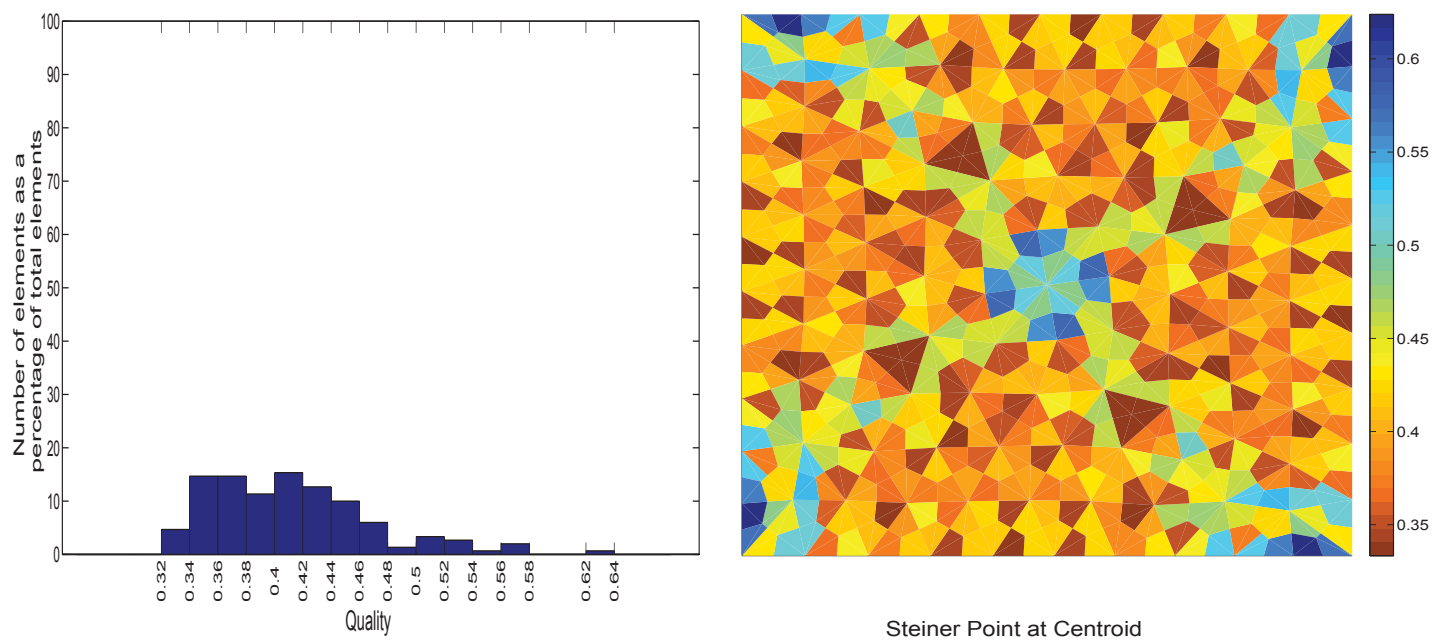

(a)
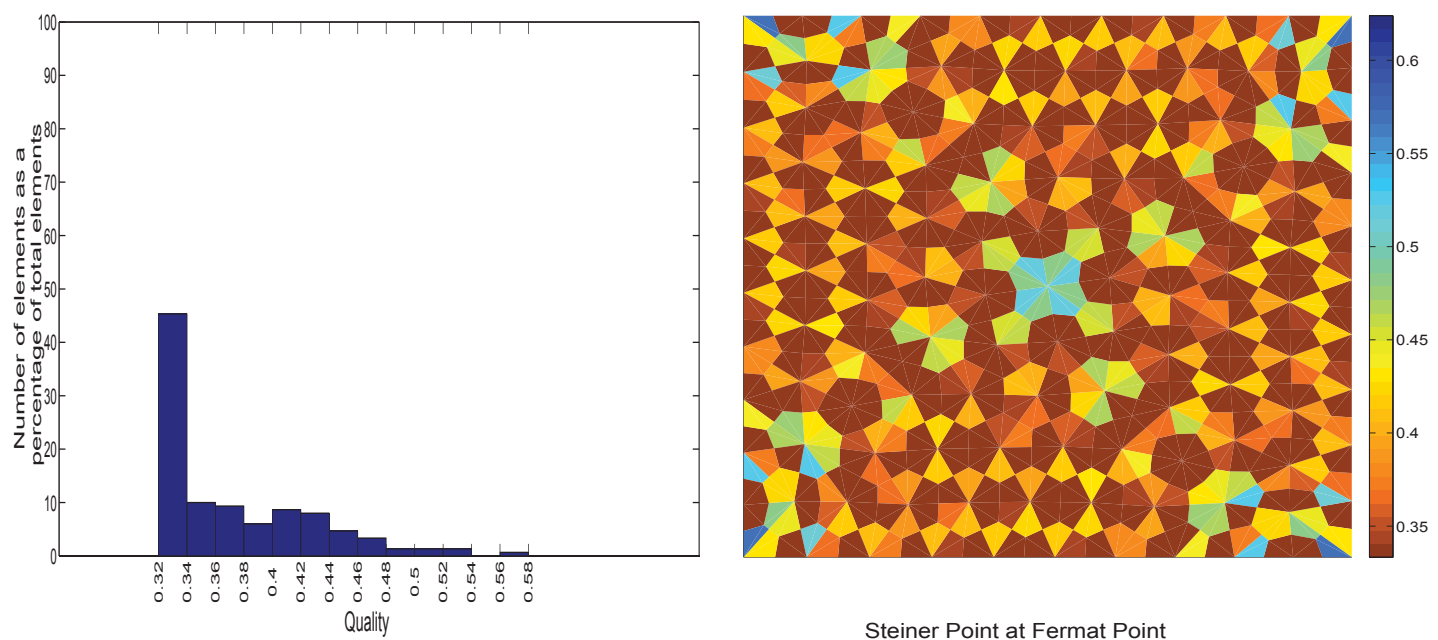

(b)

Figure 5.15: A histogram and a color bar using an Equi-angle skew quality metric to compare the tri-quadrilateralization achieved by a) Using the centroid as Steiner Point and b)Using the Fermat point as Steiner point

seen that the number of higher quality elements has increased when using the Fermat point instead of the centroid for quadrilateralization of the triangular mesh. 

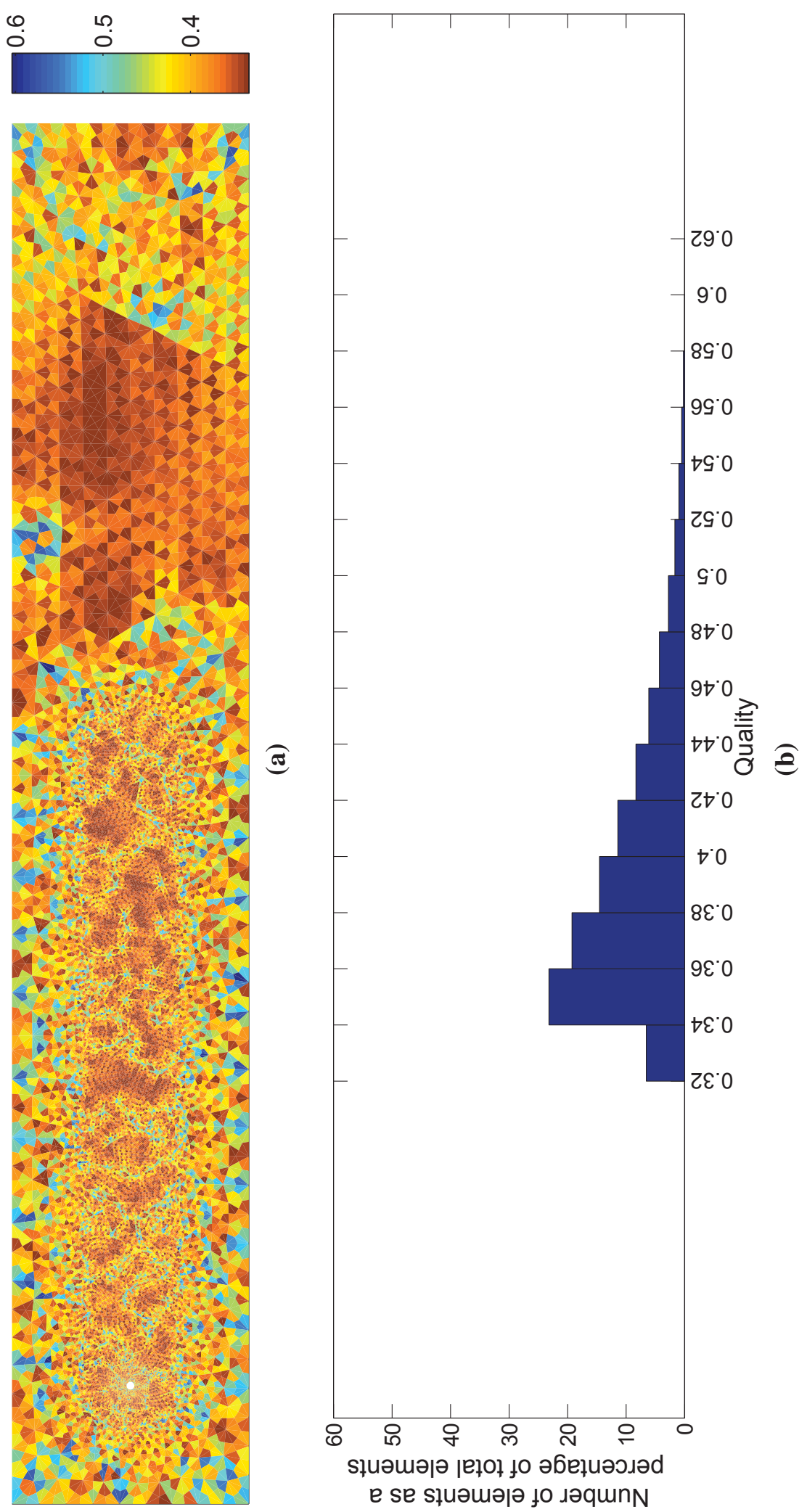

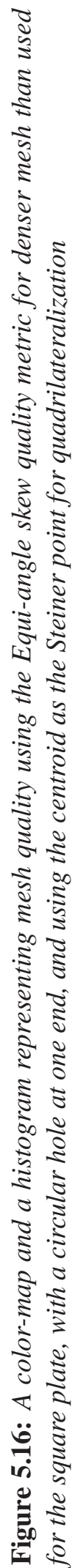



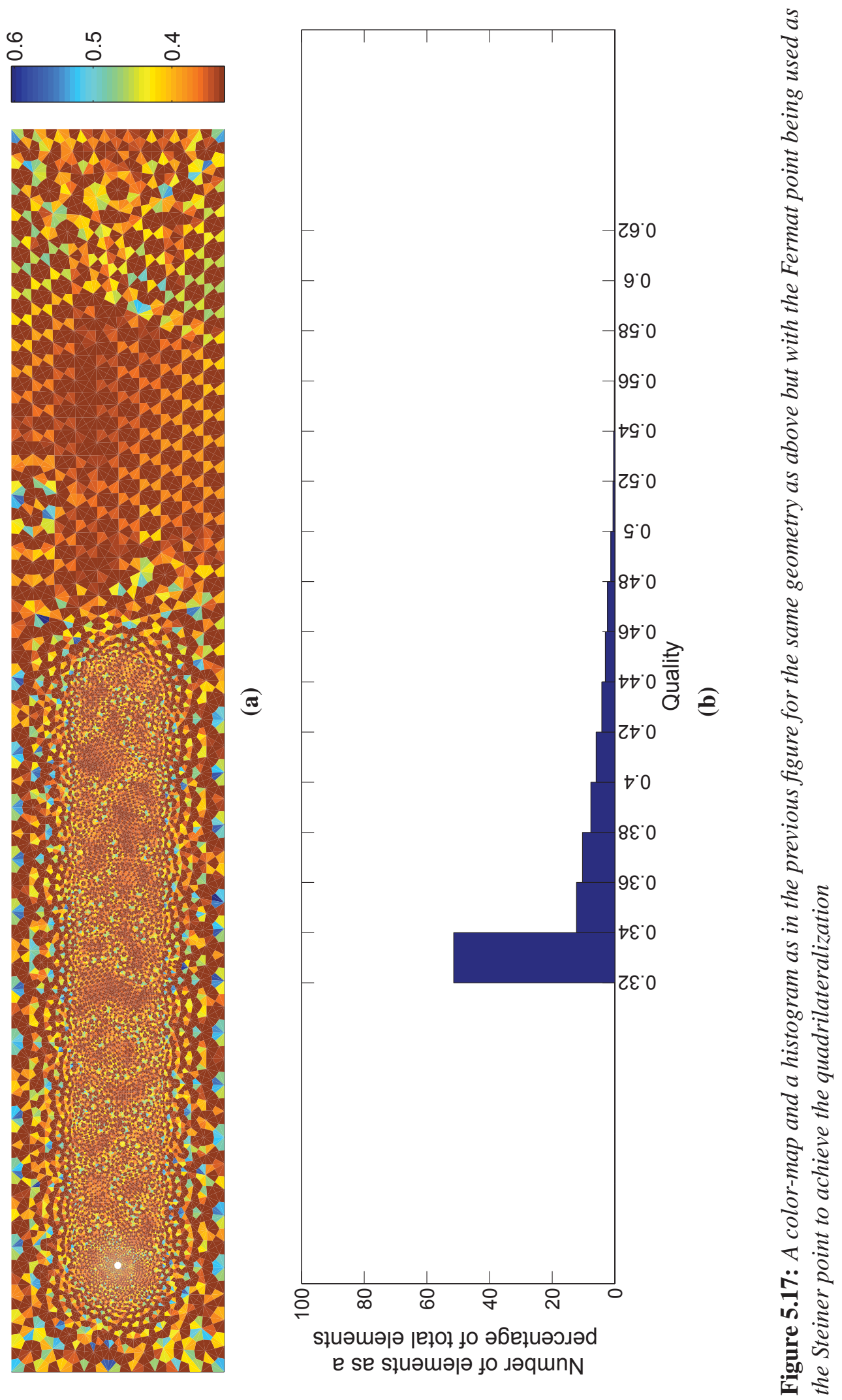

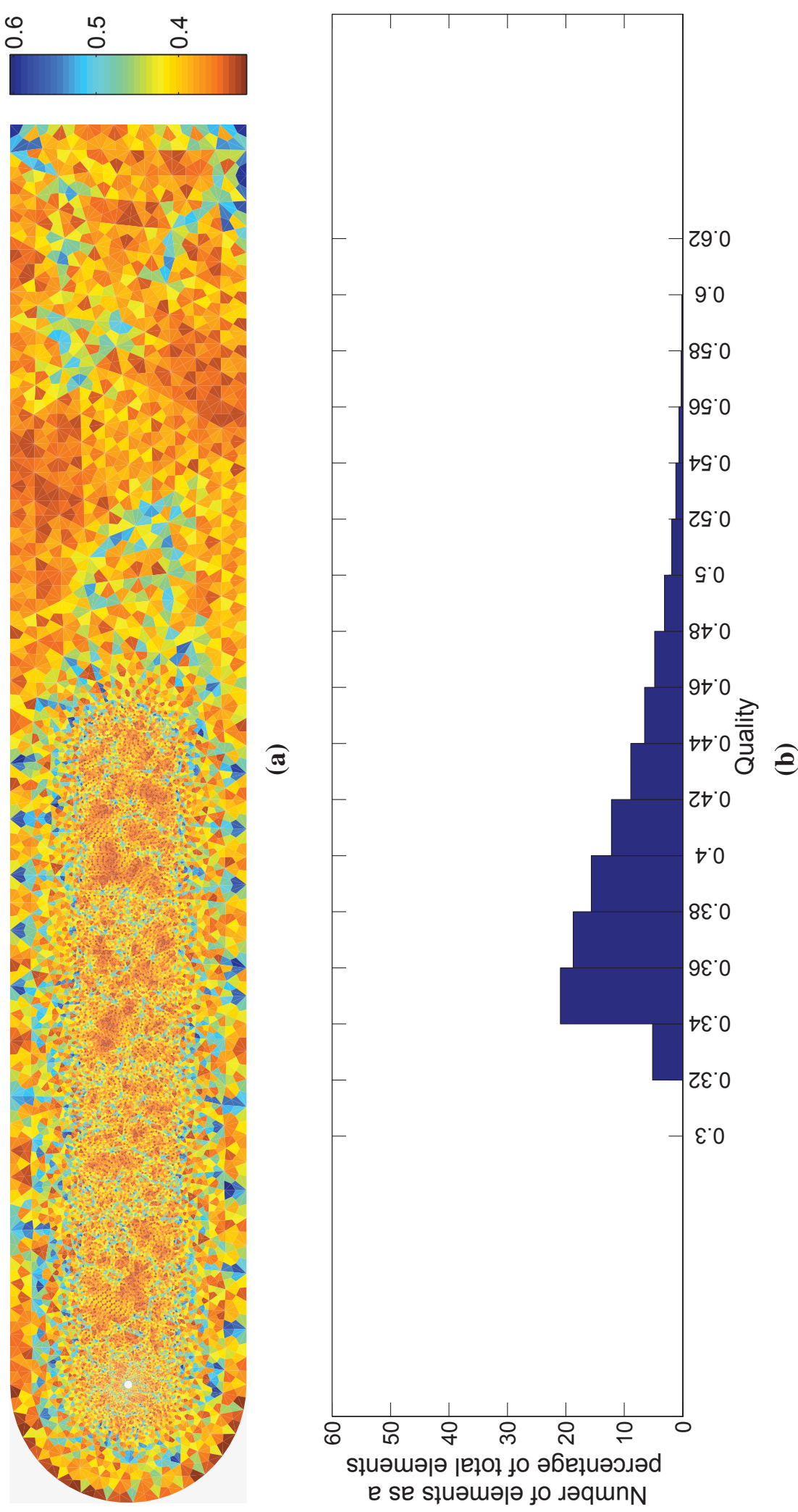

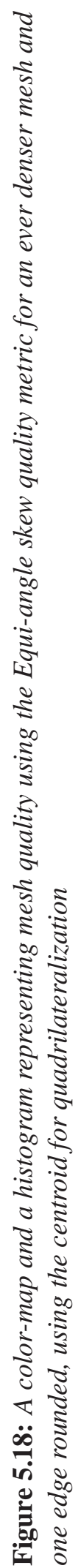



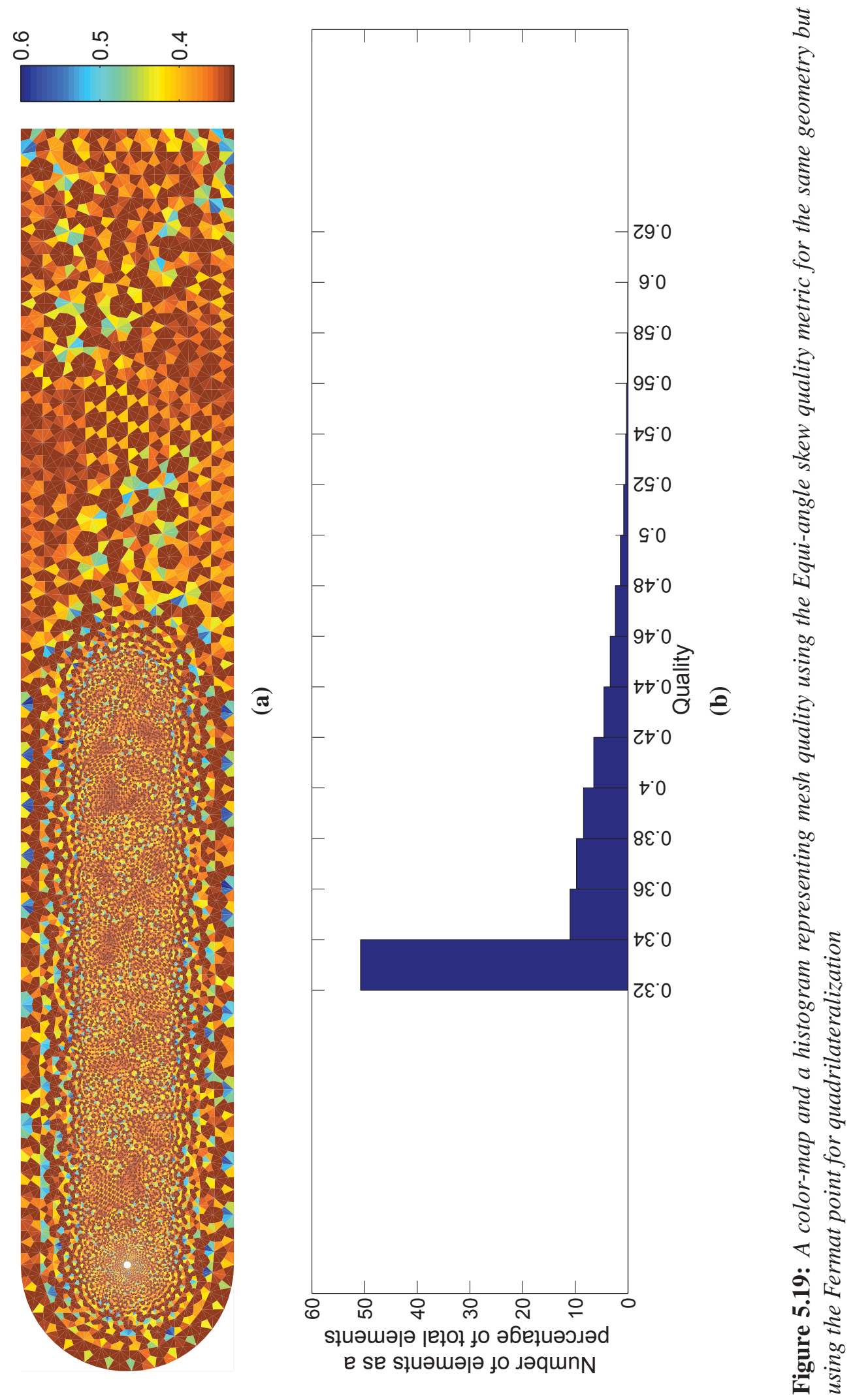


\section{Appendix A}

\section{Non-fractional step methods}

This Appendix gives a brief outline of some of the more popular Non-fractional step methods using the primimtive variables formulation. It should be noted that from now on the above linearized temporal discretization formulation will be written without the superscripts " $n$ " for time, but will imply the same.

Therefore, the problem (1.5) can be represented as

$$
\begin{array}{r}
(-\Delta+\gamma) \mathbf{u}+\nabla p=\mathbf{g} \\
-\nabla^{2} p=-\boldsymbol{\nabla} \cdot \mathbf{g} \\
\left.\mathbf{u}\right|_{S}=\mathbf{b} \text { and }\left.\boldsymbol{\nabla} \cdot \mathbf{u}\right|_{S}=0 \\
\text { given the Global Constraint } \oint_{S} \mathbf{n} \cdot \mathbf{u}_{S} \cdot d S
\end{array}
$$

\section{The Glowinski and Pironneau method}

This method uses a velocity potential function to help enforce the continuity equation and find a pressure boundary condition. The basic idea is to find a boundary condition $\lambda$ for the pressure that would give a velocity field satisfying the continuity condition at the boundary. Considering first, equation (1.5), the method first decomposes the velocity to get a unique 
function $\theta$ (42) such that

$$
\begin{array}{r}
\mathbf{u}=\boldsymbol{\nabla} \theta+\boldsymbol{\nabla} \times \boldsymbol{\Psi} \\
\left.\theta\right|_{S}=0
\end{array}
$$

Taking the divergence of both sides of the above equation gives a poisson's equation

$$
\begin{array}{r}
-\nabla^{2} \theta=-\boldsymbol{\nabla} \cdot \mathbf{u} \\
\left.\theta\right|_{S}=0
\end{array}
$$

On applying the operator $[-\Delta+\gamma \mathbf{I}]$ to the above equation one gets from the divergence of momentum equation and the poisson pressure equation $[-\Delta+\gamma \mathbf{I}] \nabla^{2} \theta=0$. If for this equation boundary conditions are given $\left.\theta\right|_{S}=0$ and $\left.\frac{\partial \theta}{\partial n}\right|_{S}=0$ are given the solution for $\theta$ would be 0 . Clearly this implies from $-\nabla^{2} \theta=-\boldsymbol{\nabla} \cdot \mathbf{u}$ that $\boldsymbol{\nabla} \cdot \mathbf{u}=0$ It follows that the solution $\mathbf{u}, p$ of the following system should satisfy incompressibility conditions

$$
\begin{array}{r}
-\nabla^{2} p=-\boldsymbol{\nabla} \cdot \mathbf{g} \\
(-\Delta+\gamma) \mathbf{u}=-\boldsymbol{\nabla} p+\mathbf{g} \\
-\nabla^{2} \theta=-\boldsymbol{\nabla} \cdot \mathbf{u} \\
\left.\mathbf{u}\right|_{S}=\mathbf{b} \\
\left.\theta\right|_{S}=0 \\
\text { given that }\left.\frac{\partial \theta}{\partial n}\right|_{S}=0
\end{array}
$$

The variational form of equation (1.20) would be

$$
\oint \frac{\partial \theta}{\partial n} \mu S=0
$$


where $\mu$ is an arbitrary function on the boundary.Thus, we must find $\lambda$ such that equation (A-5) is satisfied.

Using the principle of superposition the solutions of $\mathbf{u}, p$ and $\theta$ can be split into a harmonic and non harmonic part giving

$$
\begin{aligned}
\mathbf{u}(\mathbf{x}) & =\mathbf{u}^{0}+\oint \mathbf{u}^{6}\left(\mathbf{x} ; \zeta^{6}\right) \lambda\left(\zeta^{6}\right) d S\left(\zeta^{\prime}\right) \\
p(\mathbf{x}) & =p^{0}+\oint p^{6}\left(\mathbf{x} ; \zeta^{6}\right) \lambda\left(\zeta^{6}\right) d S\left(\zeta^{\prime}\right) \\
\theta(\mathbf{x}) & =\theta^{0}+\oint \theta^{\bullet}\left(\mathbf{x} ; \zeta^{\natural}\right) \lambda\left(\zeta^{6}\right) d S\left(\zeta^{\prime}\right)
\end{aligned}
$$

for $\zeta^{\prime} \in S$ such that $p^{0}, \mathbf{u}^{0}$ and $\theta^{0}$ are solutions to

$$
\begin{array}{r}
-\nabla^{2} p^{0}=-\boldsymbol{\nabla} \cdot \mathbf{g} \text { where }\left.p^{0}\right|_{S}=0 \\
(-\Delta+\gamma) \mathbf{u}^{0}=-\boldsymbol{\nabla} p^{0}+\mathbf{g} \text { where }\left.\mathbf{u}^{0}\right|_{S}=\mathbf{b} \\
-\nabla^{2} \theta^{0}=-\boldsymbol{\nabla} \cdot \mathbf{u}^{0} \text { where }\left.\theta^{0}\right|_{S}=0
\end{array}
$$

and the functions with the prime superscript solve

$$
\begin{gathered}
-\nabla^{2} p^{\natural}=0 \text { where }\left.p^{\natural}\right|_{S}=\delta\left(s-\zeta^{\prime}\right) \\
(-\Delta+\gamma) \mathbf{u}^{\natural}=-\boldsymbol{\nabla} p^{\natural} \text { where }\left.\mathbf{u}^{\natural}\right|_{S}=0 \\
-\boldsymbol{\nabla}^{2} \theta^{\natural}=-\boldsymbol{\nabla} \cdot \mathbf{u}^{\text {' }} \text { where }\left.\theta^{\natural}\right|_{S}=0
\end{gathered}
$$

where $\delta$ is a dirac function on the boundary these set of equations in an FEA analysis, can be reduced to a linear equation of the type $\mathbf{A} \boldsymbol{\lambda}=\boldsymbol{\beta}$ using the condition (A-6) Which can be solved for $\lambda$ and hence the poisson's problem can be approximated. The method is as big as the number of grid points on the boundary from a numerical aspect. 


\section{Influence Matrix Method}

The influence matrix techniques, like the Glowinski Pironneau method, are based on the principle of superposition for linear problems. The linearized elliptic problems with unknown boundary conditions are split into a set of relatively simpler problems with known boundary conditions. Here the linearity has been assured by the semi discretization in time giving a set of linear elliptic partial differential equations. This has already been shown in equation (1.1). The following discussion is only for two dimensional flows. Though it can easily be extended into three dimensional flows, it does require a lot of memory and hence not the best option for three dimensional problems.

Therefore, considering the set of equations (1.15), the solution for velocity and pressure are split into their homogeneous and particular solution parts as follows

$$
\begin{gathered}
\mathbf{u}=\overline{\boldsymbol{u}}+\sum_{k=1}^{N_{b}} \lambda_{k} \hat{\boldsymbol{u}}_{\boldsymbol{k}} \\
p=\bar{p}+\sum_{k=1}^{N_{b}} \lambda_{k} \hat{p}_{k}
\end{gathered}
$$

Where $\lambda_{k} \mathrm{~s}$ are determined by enforcing the incompressibility constraint on the boundary and $N_{b}$ is the number of boundary points or the number of degrees of freedom on the boundary

The particular solution problem is

$$
\begin{array}{r}
(-\Delta+\gamma) \overline{\boldsymbol{u}}+\nabla \bar{p}=\mathbf{g} \text { in } \Omega \\
\overline{\boldsymbol{u}}=0 \text { in } S \\
\nabla^{2} \bar{p}=\nabla \cdot \mathbf{g} \text { in } \Omega \quad \text { such that } \bar{p}_{k}=\bar{p}_{S} \text { in } S
\end{array}
$$


where $\bar{p}_{S}$ is an arbitrary dirichlet distribution of pressure at the boundary mostly taken to be zero.

The homogeneous problem is

$$
\begin{array}{r}
(-\Delta+\gamma) \hat{\boldsymbol{u}}+\nabla \hat{p}=0 \text { in } \Omega \\
\hat{\boldsymbol{u}}=0 \text { in } S \\
\nabla^{2} \hat{p}=0 \text { in } \Omega \\
\hat{p}_{k}=\delta_{k j}
\end{array}
$$

where $k, j$ are nodes on the boundary.

The velocity field $\hat{\boldsymbol{u}}$ is then determined from the solution to the homogeneous problem (A-9). For each boundary point and owing to the pressure distribution equal to the dirac function a matrix of the order $N_{b} \times N_{b}$ is obtained. This is in fact the influence matrix. Another way to look at it would be to see that for both the the split equations for pressure as well as velocity the coefficient lambda is the same. So the idea is to find lambda using the split equation for velocity (A-7) such that the incompressibility condition is satisfied at the boundary and then use it to get the correct pressure boundary values. Taking the divergence of both sides of the split equation for velocity (A-7) Since $\boldsymbol{\nabla} \cdot \mathbf{u}=0$

therefore, $\boldsymbol{\nabla} \cdot \overline{\boldsymbol{u}}_{j}=-\sum_{k=1}^{N_{b}} \lambda_{k} \hat{\boldsymbol{u}}_{\boldsymbol{k}}$

therefore, $\boldsymbol{\lambda}=\mathrm{A}^{-1} \mathbf{f}$

where, $\mathbf{A}$ is the $N_{b} \times N_{b}$ influence matrix and $\mathbf{f}$ is a column matrix with $N_{b}$ elements such that $f_{j}=\nabla \cdot \overline{\boldsymbol{u}}_{j}$ 
$\hat{\boldsymbol{u}}$ can be found by solving the problem (A-10).

Therefore, the correct boundary condition for pressure would be $p_{S}=\bar{p}_{S}+\lambda_{k}$.

This boundary condition is then used to calculate the correct pressure and velocity field from the equation (A-1). Note, however, that the divergence free boundary condition has already been satisfied.

\section{The integral constraint}

The idea for an integral constraint on pressure was put forth by Quartapelle and Napolitano(11).

The following discussion is based entirely on their paper on the same. Their basic aim was to come up with a method which would ensure the decoupling of the pressure and velocity by deriving pressure conditions not depending on the internal velocity field but only on the previously calculated velocity field and the boundary velocity For this, the vector equivalent of the Green's identity for the helmholtz operator $(-\Delta+\gamma)$ is considered, which is ultimately shown to imply the following integral constraint on pressure

$$
\int(-\nabla p+\mathbf{g}) \cdot \boldsymbol{\eta} d \Omega=\oint(\mathbf{n} \times \mathbf{b} \cdot \nabla \times \boldsymbol{\eta}+\mathbf{n} \cdot \mathbf{b} \nabla \cdot \boldsymbol{\eta}) d S
$$

where $\boldsymbol{\eta}$ is a harmonic field in the domain and a solution to the following problem:

$$
\begin{array}{r}
(-\Delta+\gamma) \boldsymbol{\eta}=0 \\
\mathbf{n} \times\left.\boldsymbol{\eta}\right|_{S}=0 \\
\left.\mathbf{n} \cdot \boldsymbol{\eta}\right|_{S}=0
\end{array}
$$


Thus the integral condition on pressure depends only on the boundary velocity $\mathbf{b}$ and the source term $\mathrm{g}$ owing to the explicit advection equation. The number of linearly independant $\boldsymbol{\eta} s$ fields is the same as the number of boundary points, since the problem in $\boldsymbol{\eta}$ involves solving equations equal to the number of boundary nodes therefore, the following well posed problem can be written as an uncoupled formulation of equation (A-1)

$$
\begin{array}{r}
-\nabla^{2} p=-\boldsymbol{\nabla} \cdot \mathbf{g} \\
\int(-\nabla p+\mathbf{g}) \cdot \boldsymbol{\eta} d \Omega=\oint(\mathbf{n} \times \mathbf{b} \cdot \boldsymbol{\nabla} \times \boldsymbol{\eta}+\mathbf{n} \cdot \mathbf{b} \boldsymbol{\nabla} \cdot \boldsymbol{\eta}) d S \\
(-\Delta+\gamma) \boldsymbol{\eta}=0 \\
\mathbf{n} \times\left.\boldsymbol{\eta}\right|_{S}=0 \\
\left.\mathbf{n} \cdot \boldsymbol{\eta}\right|_{S}=0 \\
(-\triangle+\gamma) \mathbf{u}+\boldsymbol{\nabla} p=\mathbf{g} \\
\left.\mathbf{u}\right|_{S}=\mathbf{b}
\end{array}
$$

This time discretized formulation can be discretized in space by any suitable method namely, finite elements, finite difference, or spectral methods. A possible solution technique similar to the influence matrix method was presented in (11). It starts with the decomposition of the pressure at a time step $(n+1)$ into a harmonic and non-harmonic component as follows

$$
p(\mathbf{x})=p^{0}(\mathbf{x})+\oint p^{6}\left(\mathbf{x} ; \zeta^{6}\right) \lambda\left(\zeta^{6}\right) d S\left(\zeta^{\prime}\right)
$$

for $\zeta^{\prime} \in S$ such that

$\nabla^{2} p^{6}=\left.0 \quad p^{\prime}\right|_{S}=\delta\left(s-\zeta^{\prime}\right)$,

and $\nabla^{2} p^{0}=-\left.\boldsymbol{\nabla} \cdot \mathrm{g} \quad p^{0}\right|_{S}=p_{S}^{0}$, where $p_{S}^{0}$ is arbitrary 
$\delta$ is the dirac function over the boundary

Imposing on the pressure the integral constraint (A-12) gives a linear equation.

$\mathrm{A} \lambda=\boldsymbol{\beta}$

This can be used to determine $\lambda$ which eventually gives the boundary condition for pressure just as in the influence matrix technique. This pressure can be used to solve for the velocity field in the following formulation

$$
\begin{array}{r}
-\nabla^{2} p=-\left.\nabla \cdot \mathbf{g} \quad p\right|_{S}=\lambda \\
(-\Delta+\gamma) \mathbf{u}+\nabla p=\mathbf{g} \\
\left.\mathbf{u}\right|_{S}=\mathbf{b}
\end{array}
$$




\section{Appendix B}

\section{The Influence matrix technique for hybrid formulations}

This technique is very similar to the one used in velocity pressure formulation starting with splitting vorticity and velocity into the homogeneous and the particular solution part as follows

$$
\begin{aligned}
& \boldsymbol{u}=\overline{\boldsymbol{u}}+\sum_{k=1}^{N_{b}} \lambda_{k} \hat{\boldsymbol{u}}_{\boldsymbol{k}} \\
& \omega=\bar{\omega}+\sum_{k=1}^{N_{b}} \lambda_{k} \hat{\omega}_{k}
\end{aligned}
$$

Where $\lambda$ is determined by enforcing on the boundary $\boldsymbol{\nabla} \times \boldsymbol{u}=\omega \boldsymbol{k}$ and Nb is the degree of freedom or the number of nodes on the boundary. The two problems can be defined separately as

1. Homogeneous problem

$$
\begin{array}{rr}
\left(-\nabla^{2}+\gamma\right) \hat{\omega}_{k}^{n+1}=0 & \text { in } \Omega \\
\hat{\omega}_{k}=\delta_{k j} & \text { on } S \\
\nabla^{2} \hat{\boldsymbol{u}}_{k}^{n+1}=-\boldsymbol{\nabla} \times\left(\hat{\omega}_{k}^{n+1} \boldsymbol{k}\right) & \text { in } \Omega \\
\hat{\boldsymbol{u}}^{n+1}=0 & \text { on } S
\end{array}
$$


2. Particular solution problem

$$
\begin{array}{rr}
\left(-\nabla^{2}+\gamma\right) \bar{\omega}_{k}^{n+1}=f & \text { in } \Omega \\
\bar{\omega}_{k}=\bar{\omega}_{S} & \text { on } S \\
\nabla^{2} \overline{\boldsymbol{u}}^{n+1}=-\nabla \times\left(\bar{\omega}^{n+1} \boldsymbol{k}\right) & \text { in } \Omega \\
\hat{\boldsymbol{u}}^{n+1}=0 & \text { on } S
\end{array}
$$

Where $\bar{\omega}_{S}$ is an arbitrary value usually taken as zero

$\boldsymbol{\nabla} \times \boldsymbol{u}=\omega \boldsymbol{k}$ can be enforced on the boundary using the following equation

$$
(\hat{\eta}-\hat{\omega})\left(\gamma_{j}\right)+\sum_{k=1}^{N_{b}} \boldsymbol{\lambda}_{k}\left(\hat{\eta}_{k}-\hat{\omega}_{k}\right)\left(\gamma_{j}\right)=0, \text { Where } j=1,2 \ldots N_{b}, \quad \gamma_{j} \in N_{b}
$$

Where, $\bar{\eta}=(\boldsymbol{\nabla} \times \overline{\boldsymbol{u}}) \cdot \boldsymbol{k}$ and $\hat{\eta}_{k}=\left(\boldsymbol{\nabla} \times \hat{\boldsymbol{u}}_{k}\right) \cdot \boldsymbol{k}$

Giving the following linear problem $\boldsymbol{A \lambda}=\boldsymbol{f}$

for $\boldsymbol{\lambda}$ Where, $\boldsymbol{A}$ is a $N_{b} \times N_{b}$ matrix given by $\left(\hat{\eta}_{k}-\hat{\omega}_{k}\right)\left(\gamma_{j}\right)$ and $\boldsymbol{f}$ is a $N_{b} \times 1$ column matrix given by $(\hat{\eta}-\hat{\omega})\left(\gamma_{j}\right)$ The boundary value of vorticity is then given by

$$
\left(\omega_{S}\right)_{k}=\left(\bar{\omega}_{S}\right)_{k}+\lambda_{k}
$$

For problems with very low viscosity this method shows a lot of numerical instability. A possible modification has been suggested by Clercx (43)

An uncoupled formulation can be written by imposing the following integral constraint for vorticity

$$
\int \nabla \omega \times \boldsymbol{k} \cdot \boldsymbol{\eta} d \Omega=\oint(\boldsymbol{n} \times \boldsymbol{b} \cdot \boldsymbol{\nabla} \times \boldsymbol{\eta}+\boldsymbol{n} \cdot \boldsymbol{b} \nabla \cdot \boldsymbol{\eta}) \cdot d S
$$


Where, $\eta$ is the same as descirbed for three dimensional flow. Thus, an uncoupled formulation can be written by imposing the integral constraint for vorticity as follows

$$
\begin{array}{rr}
\left(\frac{\partial \omega}{\partial t}-\nu \nabla^{2} \omega\right) \boldsymbol{k}+\boldsymbol{\nabla} \times(\omega \boldsymbol{k} \times \boldsymbol{u})=0, & \omega_{t=0}=\boldsymbol{\nabla} \times \boldsymbol{u}_{0} \cdot \boldsymbol{k} \\
\int \boldsymbol{\nabla} \omega \times \boldsymbol{k} \cdot \boldsymbol{\eta} d \Omega=\oint(\boldsymbol{n} \times \boldsymbol{b} \cdot \boldsymbol{\nabla} \times \boldsymbol{\eta}+\boldsymbol{n} \cdot \boldsymbol{b} \boldsymbol{\nabla} \cdot \boldsymbol{\eta}) \cdot d S & \int \omega d \Omega=\oint \boldsymbol{\tau} \cdot \boldsymbol{b} d S \\
-\boldsymbol{\nabla}^{2} \boldsymbol{\eta}=\left.0 \quad \boldsymbol{\tau} \cdot \boldsymbol{\eta}\right|_{S}=0 \\
-\nabla^{2} \boldsymbol{u}=\boldsymbol{\nabla} \omega \times \boldsymbol{k}
\end{array}
$$

$\int \omega d \Omega=\oint \boldsymbol{\tau} \cdot \boldsymbol{b} d S$ is obtained by integrating $\boldsymbol{\nabla} \times \boldsymbol{u}=\omega \boldsymbol{k}$ over the entire domain.

Using the time discretized scheme derived above the above equation can be linearized as follows

$$
\begin{array}{r}
\left(-\nabla^{2}+\gamma\right) \omega=f, \quad \int \omega d \Omega=\oint \boldsymbol{\tau} \cdot \boldsymbol{b} d S \\
\int \boldsymbol{\nabla} \omega \times \boldsymbol{k} \cdot \boldsymbol{\eta} d \Omega=\oint(\boldsymbol{n} \times \boldsymbol{b} \cdot \boldsymbol{\nabla} \times \boldsymbol{\eta}+\boldsymbol{n} \cdot \boldsymbol{b} \boldsymbol{\nabla} \cdot \boldsymbol{\eta}) . d S \\
-\nabla^{2} \boldsymbol{u}=\boldsymbol{\nabla} \omega \times\left.\boldsymbol{k} \quad \boldsymbol{u}\right|_{S}=\boldsymbol{b}
\end{array}
$$

Here the superscripts for the time steps has not been written for simplicity.

A possible way to implement this technique is for the two dimensional case is the GlowinskiPironneau Method. As before $\omega$ can be decomposed using the principle of superposition to give

$$
\omega(\boldsymbol{x})=\omega^{0}(\boldsymbol{x})+\oint \omega^{6}\left(\boldsymbol{x} ; \zeta^{6}\right) \lambda\left(\zeta^{6}\right) d S\left(\zeta^{\prime}\right)
$$


Where, $\omega^{0}$ and, $\omega^{6}$ are the solutions to

$$
\begin{array}{r}
(-\Delta+\gamma) \omega^{0}=\left.f \quad \omega^{0}\right|_{S}=0 \\
(-\Delta+\gamma) \omega^{6}=0 \quad \omega^{6}\left(\boldsymbol{x} ; \zeta^{6}\right)=\delta\left(s-\zeta^{6}\right)
\end{array}
$$

for any $\zeta^{6} \in S$ and $\delta$ is the dirac delta function over the boundary.

Again, giving a linear problem of the sort $\boldsymbol{A} \boldsymbol{\lambda}=\boldsymbol{\beta}$. This combined with another linear problem of similar type using the second condition equation (2.22) gives the value of $\lambda$ at the boundary which can be used to supplement the boundary value of vorticity. 


\section{Appendix C}

Permission for reusing the image in figure 1.1

This file is licensed under the Creative Commons Attribution-Share Alike 3.0 Unported license. You are free:

1. to share to copy, distribute and transmit the work

2. to remix to adapt the work

Under the following conditions:

1. attribution You must attribute the work in the manner specified by the author or licensor (but not in any way that suggests that they endorse you or your use of the work).

2. share alike If you alter, transform, or build upon this work, you may distribute the resulting work only under the same or similar license to this one.

\section{ATTRIBUTION}

Hans Hillewaert / CC-BY-SA-3.0 


\section{References}

[1] Williamson CHK, Govardhan R. Vortex-induced vibrations. Annual Review of Fluid Mechanics . 2004;36:413-455.

[2] Ponta FL. The kinematic Laplacian equation method. Journal of Computational Physics . 2005;207:405-426.

[3] Fasel H. Investigation of the stability of boundary layers by a finite difference model of the Navier-Stokes equations. Journal of Fluid Mechanics . 1976;78:355-383.

[4] Clercx HJH. A spectral solver for the Navier-Stokes equations in the velocityvorticity formulation for flows with two nonperiodic directions. Journal of Computational Physics . 1997;137:186-211.

[5] Hillewaert H. Windmills D1-D4 (Thornton Bank). http://en.wikipedia. org/wiki/File:Windmills_D1-D4_(Thornton_Bank).jpg. 2009. See Appendix for documentation of permission to republish this material.

[6] NREL. Wind Power Today. Report DOE/GO-102005-2115, U.S. Department of Energy. 2005. 
[7] de Vries E. Thinking bigger: Are there limits to turbine size? Renewable Energy World . 2005;8(3).

[8] Rempfer D. On Boundary Conditions for Incompressible Navier-Stokes Problems. Applied Mechanics Reviews . 2006;59:107-125.

[9] Glowinski R. Finite Element Methods for Navier-Stokes equations. Annual Review of Fluid Mechanics . 1992;24:107-125.

[10] Quartapelle L. Numerical solution of the incompressible Navier-Stokes equations. Basel, Switzerland: Birkäuser. 1993.

[11] Quartapelle L, Napolitano M. Integral Conditions for the pressure in the computation of Incompressible viscous flows. Journal of Computational Physics . 1986;62:340_ 348.

[12] Fletcher C. Computational Techniques for Fluid Dynamics Vol.2. 2nd ed. Springer. 1988.

[13] Kwak D, Kiris C, Kim CS. Computational challenges of viscous incompressible flows. Computers and Fluids . 2005;34:283-299.

[14] Kempka S, Strickland J, Glass M, Peery J, Ingber PM. Velocity Boundary Conditions for Vorticity Formulations of the Incompressible Navier-Stokes Equations. In: Forum on the Application of Vortex Methods to Engineering Problems. The U.S. Dept. of Energy. 
[15] Gatski TB. Review of incompressible fluid flow computations using the vorticityvelocity formulation. Applied Numerical Mathematics . 1991;7:227-239.

[16] Davies C, Carpenter PW. A Novel Velocity-Vorticity Formulation of the Navier-Stokes Equations with Applications to Boundary Layer Disturbance Evolution. Journal of Computational Physics . 2001;172:119-165.

[17] X H Wu JZW, Wu JM. Effective vorticity-velocity formulations for 3D incompressible viscous flows. Journal of Computaional Physics . 1995;122:68-82.

[18] Karniadakis GE, Sherwin S. Spectral/hp Element Methods for CFD. 2nd ed. Oxford University Press. 2005.

[19] Ponta FL. Kinematic Laplacian Equation Method: A Velocity-Vorticity Formulation for the Navier-Stokes Equations. Journal of Applied Mechanics . 2006;73:1031-1038.

[20] Ponta FL, Jacovkis PM. A vortex model for Darrieus turbine using finite element techniques. Renewable Energy . 2001;24:1-18.

[21] Ponta FL, Jacovkis PM. Constant-curl Laplacian equation: a new approach for the analysis of flows around bodies. Computers and Fluids . 2003;32:975-994.

[22] Batchelor GK. An introduction to fluid dynamics. Cambridge, UK: Cambridge University Press. 2000.

[23] Anderson CR. Observations on vorticity creation boundary conditions. In: Caflisch RE, editor, Mathematical Aspects of Vortex Dynamics. SIAM. 1988;pp. 144-159. 
[24] Chorin AJ. Numerical study of slightly viscous flow. Journal of Fluid Mechanics . 1973;57:785-796.

[25] Chorin AJ. Vortex sheet approximation of boundary layers. Journal of Computational Physics . 1978;27:428-442.

[26] Boyd JP. Chebyshev and Fourier spectral methods. Mineola, New York, USA: Dover. 2000.

[27] Sherwin SJ, Karniadakis GE. Tetrahedral hp Finite Elements: Algorithms and Flow Simulations. Journal of Computational Physics . 1996;124:14-45.

[28] Thompson MC, Hourigan K, Sheridan J. Three-dimensional instabilities in the wake of a circular cylinder. Experimental Thermal and Fluid Science . 1996;12:190-196.

[29] Karniadakis GE, Israeli M, Orszag SA. High-Order Splitting Methods for the Incompressible Navier-Stokes Equations. Journal of Computational Physics . 1991; 97:414-443.

[30] Sprague MA, Geers TL. Legendre spectral finite elements for structural dynamics analysis. Communications in Numerical Methods in Engineering . 2007;00:1-13.

[31] Patera AT. A spectral element method for fluid dynamics: laminar flow in a channel expansion. Journal of Computational Physics . 1984;54:468-488.

[32] Karniadakis GE, Bullister ET, Patera AT. A spectral element method for solution of two- and three-dimensional time-dependent incompressible Navier-Stokes equations. 
In: Finite Element Methods for Nonlinear Problems. New York/Berlin: SpringerVerlag, p. 803.

[33] Henderson RD, Karniadakis GE. Unstructured spectral element methods for simulation of turbulent flows. Journal of Computational Physics . 1995;122:191-217.

[34] Hourigan K, Thompson MC, Tan BT. Self-sustained oscilations in flows around long blunt plates. Journal of Fluids and Structures . 2001;15:387-398.

[35] Giraldo FX. The Lagrange-Galerkin spectral element method on unstructured quadrilateral grids. Journal of Fluid Mechanics . 1998;147:114-146.

[36] Bathe KJ. Finite element procedures. Englewood Cliffs, New Jersey, USA: Prentice Hall. 1996.

[37] Pathria D, Karniadakis GE. Spectral element method for elliptic problems in nonsmooth domains. Journal of Computational Physics . 1994;122:83-95.

[38] Knupp PM. Remarks on Mesh Quality. In: 45th AIAA Aerospace Sciences Meeting and Exhibit. American Institute of Aeronautics and Astronautics.

[39] Knupp PM. Algebraic mesh quality metrics for unstructured initial meshes. Finite Elements in Analysis and Design . 2003;39:217-241.

[40] Gambit 2.4 Documentation. Fluent inc.

[41] Varilly A. Location of Incenters and Fermat Points in Variable Triangles . 2001; 
[42] Waters LK, Fix GJ, Cox CL. The Method of Glowinski and Pironneau for the Unsteady Stokes Problem. Computers and Mathematics with Applications . 2004; 48:1191-1211.

[43] Clercx HJH. A Spectral Solver for the Navier-Stokes Equations in the Velocity-Vorticity Formulation for Flows with Two Nonperiodic Directions. Journal of Computational Physics . 1997;137:186-211. 